August 2017

\title{
Diabetes in sub-Saharan Africa: from clinical care to health policy.
}

Rifat Atun

Harvard University

Justine I. Davies

University of Witwatersrand

Edwin A.M. Gal

University of Bristol

Till Bärnighausen

Harvard University

David Beran

University of Geneva

See next page for additional authors

Follow this and additional works at: https://ecommons.aku.edu/ eastafrica_fhs_mc_paediatr_child_health

Part of the Pediatrics Commons

\section{Recommended Citation}

Atun, R., Davies, J. I., Gal, E. A., Bärnighausen, T., Beran, D., Kengne, A. P., Levitt, N. S., Mangugu, F., Nyirenda, M. J., Ogle, G. D., Ramaiya, K. (2017). Diabetes in sub-Saharan Africa: from clinical care to health policy.. Lancet Diabetes and Endocrinology, 5(8), 622-667.

Available at: https://ecommons.aku.edu/eastafrica_fhs_mc_paediatr_child_health/142 
Authors

Rifat Atun, Justine I. Davies, Edwin A.M. Gal, Till Bärnighausen, David Beran, Andre Pascal Kengne, Naomi S. Levitt, Florence Mangugu, Moffat J. Nyirenda, Graham D. Ogle, and Kaushik Ramaiya 


\section{The Lancet Diabetes \& Endocrinology Commission}

\section{Diabetes in sub-Saharan Africa: from clinical care to health policy}

Rifat Atun*, Justine I Davies*, Edwin A M Gale*, Till Bärnighausen, David Beran, Andre Pascal Kengne, Naomi S Levitt, Florence W Mangugu, Moffat J Nyirenda, Graham D Ogle, Kaushik Ramaiya, Nelson K Sewankambo, Eugene Sobngwi, Solomon Tesfaye, John S Yudkin, Sanjay Basu, Christian Bommer, Esther Heesemann, Jennifer Manne-Goehler, Iryna Postolovska, Vera Sagalova, Sebastian Vollmer, Zulfiqarali G Abbas, Benjamin Ammon, Mulugeta Terekegn Angamo, Akhila Annamreddi, Ananya Awasthi, Stéphane Besançon, Sudhamayi Bhadriraju, Agnes Binagwaho, Philip I Burgess, Matthew J Burton, Jeanne Chai, Felix P Chilunga, Portia Chipendo, Anna Conn, Dipesalema RJoel, Arielle W Eagan, Crispin Gishoma, Julius Ho, Simcha Jong, Sujay S Kakarmath, Yasmin Khan, Ramu Kharel, Michael A Kyle, Seitetz C Lee, Amos Lichtman, Carl P Malm, Maïmouna N Mbaye, Marie A Muhimpundu, Beatrice M Mwagomba, Kibachio Joseph Mwangi, Mohit Nair, Simon P Niyonsenga, Benson Njuguna, Obiageli L O Okafor, Oluwakemi Okunade, Paul H Park, Sonak D Pastakia, Chelsea Pekny, Ahmed Reja, Charles N Rotimi, Samuel Rwunganira, David Sando, Gabriela Sarriera, Anshuman Sharma, Assa Sidibe, Elias S Siraj, Azhra S Syed, Kristien Van Acker, Mahmoud Werfalli

Lancet Diabetes Endocrinol 2017; 5: 622-67

Published Online July 5, 2017

http://dx.doi.org/10.1016/ S2213-8587(17)30181-X

See Comment pages 574 and 575

*Joint first authors and lead Commissioners

Harvard TH Chan School of Public Health, Harvard University, Boston, MA, USA (Prof RAtun FRCP,

ProfT Bärnighausen MD, I Postolovska SCD,

SVollmer PhD, B Ammon

A Annamreddi, A Awasthi, S Bhadriraju, J Chai MPH, J Ho BS,

SS Kakarmath MBBS MS, R Kharel, M A Kyle, SC Lee MD, A Lichtman MD,

J Manne-Goehler MD, M Nair MPH, O L O Okafor MPH

O Okunade MD, D Sando,

A Sharma MPH, A S Syed MPH);

Harvard Medical School,

Harvard University, Boston, MA, USA (Prof R Atun, A Binagwaho MD, $P$ Chipendo MD,

J Manne-Goehler); Centre for Global Health, King's College London, Weston Education Centre, London, UK

(J I Davies MD); MRC/Wits Rural Public Health and Health Transitions Research Unit, School of Public Health, Education Campus, University of Witwatersrand, Parktown, South Africa (I I Davies); University of Bristol, Bristol, UK (E A M Gale FRCP); Muhimbili University of Health and Allied

Sciences, and Abbas Medical Centre, Dar es Salaam, Tanzania (Z G Abbas MMed); Institute of Public Health, Faculty of Medicine,Heidelberg University, Heidelberg, Germany (ProfT Bärnighausen); Africa

\section{Executive summary}

Rapid demographic, sociocultural, and economic transitions are driving increases in the risk and prevalence of diabetes and other non-communicable diseases (NCDs) in sub-Saharan Africa. The impacts of these transitions and their health and economic consequences are evident. Whereas, in 1990, the leading causes of death in sub-Saharan Africa were HIV/AIDS, lower respiratory infections, diarrhoeal diseases, malaria, and vaccinepreventable diseases in children, in more recent years, cardiovascular diseases and their risk factors are replacing infectious diseases as the leading causes of death in this region, and rates of increase of cardiovascular risk factors are predicted to be greater in sub-Saharan Africa than in other parts of the world. Thus, sub-Saharan Africawhich contains a high proportion of the world's least developed countries-will face the multifaceted challenge of dealing with a high burden of infectious diseases and diseases of poverty, while also addressing the increasing burden of cardiovascular disease and its risk factors. At present, many of the health systems in sub-Saharan Africa struggle to cope with infectious diseases. Meeting the goals of the UN high-level meeting on NCDs (to reduce premature mortality from NCDs by $25 \%$ by 2025) and Sustainable Development Goals (SDGs; to reduce premature mortality from NCDs by a third by 2030) requires a coordinated approach within countries, which starts with a firm consideration of disease burden, needs, and priorities.

Diabetes is an exemplar risk factor of cardiovascular disease in that its prevalence tracks the transitions that lead to the precursors of cardiovascular disease-namely obesity and overweight. The prevalence of diabetes is increasing rapidly in sub-Saharan Africa. ${ }^{1}$ If left untreated, diabetes leads to a plethora of complications, both microvascular and macrovascular, that affect multiple physiological systems. Additionally, diabetes is closely associated with other cardiovascular risk factors, including hypertension and hypercholesterolaemia, which interact to exacerbate the risk of adverse outcomes Thus, diabetes requires an interconnected, broad-based health system for its effective management. Improving the processes of care for people with diabetes should lead to improvement of health systems for many other conditions. If left unchecked, however, the adverse outcomes of diabetes and other cardiovascular risk factors could overwhelm health systems in sub-Saharan Africa and leave many of those affected with substantial morbidity and mortality. The interaction of diabetes with infectious diseases further increases the burden of illness on resource-constrained health systems. The Lancet Diabetes \& Endocrinology Commission on Diabetes in sub-Saharan Africa was formed to ascertain the current burden of diabetes and its risk factors and outcomes in the region, to assess challenges faced by health systems in dealing with this burden, and to suggest potential solutions. We present the key messages of the Commission below and also suggest operational targets (panel 1) to help countries at all stages of development to transition to a state whereby the UN and SDG targets on NCDs can be achieved, if not surpassed.

Key message 1: the true burden of diabetes, other cardiovascular risk factors, and macrovascular and microvascular complications in sub-Saharan Africa is unknown

Estimates from those countries in which high-quality data are available suggest that the increase in the prevalence of diabetes, other cardiovascular risk factors, and adverse outcomes is large and is expected to further increase. However, most countries do not have data or data collection systems that are sufficiently reliable to enable mounting of a commensurate health-system response. To plan such a response requires high-quality, population-representative data on both current burdens and associated demographic factors and that systems for longitudinal data collection be put in place. It is also imperative to ascertain which tests and cutoffs for hyperglycaemia are most appropriate for use in defining diabetes in populations in sub-Saharan Africa to prevent overtreatment or undertreatment.

Knowledge about the burden of type 1 diabetes is particularly important given that this condition is fatal in the absence of relatively inexpensive treatment. 
Key message 2: diabetes and its consequences are costly to patients and economies

We estimate that, in 2015, the overall cost of diabetes in sub-Saharan Africa was US $\$ 19.45$ billion or $1.2 \%$ of cumulative gross domestic product (GDP). Around $\$ 10 \cdot 81$ billion $(55.6 \%)$ of this cost arose from direct costs, which included expenditure on diabetes treatment (eg, medication, hospital stays, and treatment of complications), with out-of-pocket expenditure likely to exceed $50 \%$ of the overall health expenditure in many countries. We estimate that the total cost will increase to between $\$ 35.33$ billion $(1.1 \%$ of GDP) and $\$ 59.32$ billion $(1.8 \%$ of GDP) by 2030 . Putting in place systems to prevent, detect, and manage hyperglycaemia and its consequences is therefore warranted from a health economics perspective.

\section{Key message 3: health systems in countries in} sub-Saharan Africa are unable to cope with the current burden of diabetes and its complications

By use of information from WHO Service Availability Readiness Assessment surveys and World Bank Service Delivery Indicator surveys and the local knowledge of Commissioners, we found inadequacies at all levels of the health system required to provide adequate management for diabetes and its associated risk factors and sequelae. We found inadequate availability of simple equipment for diagnosis and monitoring, a lack of sufficiently knowledgable health-care providers, insufficient availability of treatments, a dearth of locally appropriate guidelines, and few disease registries. These inadequacies result in a substantial dropoff of patients along the diabetes care cascade, with many patients going undiagnosed and with those who are diagnosed not receiving the advice and drugs they need. We also noted scarce facilities to manage the microvascular and macrovascular complications of diabetes. Additionally, despite calls for adding the care of diabetes and other cardiovascular risk factors onto existing infectious disease programmes (such as those for HIV), we found little evidence that such combined programmes are successful at improving outcomes.

\section{Key message 4: scarce health-care resources should be focused on the management of diabetes and other risk factors to prevent complications}

The management of diabetes and its risk factors is reasonably simple and inexpensive. Treating complications, however, is costly, requiring providers with a high level of skill and specialised equipment. Prevention of complications is therefore crucial. To allow effective prevention of complications, decentralisation of care-from experts who work in hospitals to community health workers and other nonclinical providers who work in the primary care system and deliver home-based screening and care-needs to be accelerated. Simple and effective information technology solutions should be used to enable more locally delivered care. An additional consideration is whether it is more beneficial to treat each risk factor associated with diabetes to predefined targets, or to consider risk factors collectively and aim to reduce overall risk. For both the prevention of macrovascular and microvascular risk factors, our analyses suggest it will be more effective and cost-effective to consider risk factors as a whole, and use benefit-based tailored treatment, rather than to treat each individual cardiovascular risk factor to a target.

Panel 1: Priorities and targets for diabetes care to 2030

When health resources are severely limited, difficult choices must often be made in the face of competing priorities. Our review of the challenges involved makes it clear that models of diabetes care for use in high-income countries are neither appropriate nor affordable in low-income or middle-income countries. We advocate the pursuit of a utilitarian approach to the provision of diabetes care in most sub-Saharan African settings, involving widely available inexpensive treatments for prevention of complications alongside strong public health measures to prevent increases in the prevalence of obesity and diabetes. Investment in preventing the consequences of diabetes will prevent the necessity of investing in wider-scale availability of expensive treatments to manage diabetes complications. Rigorous health-systems research and implementation science ${ }^{2}$ to accompany the introduction of new treatments or management strategies are key to ensuring that solutions are both fitting to a local environment and that results obtained can be of use to other countries. Funding for this type of research is urgently required.

We therefore propose a hierarchy-of-needs model of care on the basis of strategies known to work in other settings. This hierarchy is based on the Commissioners' experience in both clinical care and health-system improvement and our review of the literature during the process of this Commission. The principles of this hierarchy are straightforward: each intervention should be evidence-based, effective, accessible, integrated, and affordable. Of key importance, the Commission calls for services for provision of care and diagnostics for diabetes, its risk factors, and its complications to be fully integrated to minimise the indirect costs to the patient of having to attend multiple clinic appointments.

The prerequisites for introduction of treatment modalities or therapies are education and structure. The aims of education are achieved at personal, community, and health-care-provider levels. At a personal level, the aim is to make the patient an active, informed partner in their own therapy rather than a passive recipient. At the community level, the aim is to increase understanding and awareness of diabetes and eliminate prejudice. Education of medical personnel is needed to raise awareness of the disease and the simplicity of its treatment, and also to counterbalance marketing and medical education campaigns by the pharmaceutical industry, which are typically slanted towards use of more expensive, patented forms of treatment. An appropriate structure for health-care delivery, which is embedded in the health system, is equally essential.

We have considered necessary care needs in terms of the level of service-provision development in countries. We progress from care that we consider to be essential (which we refer to as level one care) and recommend should be available in $100 \%$ of countries by 2020 ; to level two care, which we consider should be the next step when level one care is achieved and should be available in $75 \%$ of countries by 2020 and in $100 \%$ of countries by 2025 ; and then to level three care, which should be considered once other targets have been achieved. We recommend that level three care should be present in $50 \%$ of countries by $2020,75 \%$ of countries by 2025 , and $100 \%$ of countries by 2030 (table 1 ) 


\section{Geneva, Switzerland (D Beran PhD); NGO Santé \\ Diabète, Bamako, Mali (S Besançon); Geisel School of Medicine at Dartmouth, \\ Hanover, $\mathrm{NH}$, USA (A Binagwaho); University of Global Health Equity, Kigali, \\ Rwanda (A Binagwaho); University of Goettingen,}

Key message 5: more evidence is needed about the benefits and risks (to individuals and health systems) of screening before programmes are rolled out across sub-Saharan Africa

The benefits of screening, especially in people who are deemed to be at high risk, seem obvious: earlier detection and management of diabetes and its risk factors and prevention of costly complications. However, as of yet, there is little evidence- - even from high-income countries, where studies have been done-that screening programmes are effective at reducing adverse outcomes. Additionally, the thresholds for diagnosing diabetes (ie, the level of glycaemia that is associated with the risk of adverse outcomes in the long term) and the best test to use are not defined for populations in sub-Saharan Africa. Hence, any screening programme that is started should only be done

\begin{tabular}{|c|c|c|c|}
\hline & Level one & Level two & Level three \\
\hline Target & $100 \%$ availability or uptake by 2020 & $\begin{array}{l}75 \% \text { availability or uptake by } 2020 ; 100 \% \text { availability or } \\
\text { uptake by } 2025\end{array}$ & $\begin{array}{l}50 \% \text { availability or uptake by } 2020 ; 75 \% \text { availability or } \\
\text { uptake by } 2025 ; 100 \% \text { availability or uptake by } 2030\end{array}$ \\
\hline $\begin{array}{l}\text { Medications } \\
\text { and equipment }\end{array}$ & $\begin{array}{l}\text { Availability of treatments for hyperglycaemia } \\
\text { (metformin, sulfonylureas, and short-acting and } \\
\text { intermediate-acting human insulin) at health-care } \\
\text { facilities at all levels (including community facilities and } \\
\text { pharmacies) } \\
\text { Availability of essential medicines for hypertension at all } \\
\text { health facilities (including community facilities and } \\
\text { pharmacies) } \\
\text { Availability of angiotensin-converting enzyme inhibitors } \\
\text { to treat diabetic nephropathy and prevent microvascular } \\
\text { disease at all health-care facilities (including community } \\
\text { facilities and pharmacies) } \\
\text { Availability of off-patent medicines to treat } \\
\text { hyperlipidaemia at all health-care facilities } \\
\text { Availability of blood glucose and ketone sticks at all health } \\
\text { facilities (including community health facilities) } \\
\text { Availability of working sphygmomanometers of } \\
\text { appropriate cuff size at all health facilities } \\
\text { (including community health facilities) }\end{array}$ & $\begin{array}{l}\text { Availability of essential medicines for secondary } \\
\text { prevention of cardiovascular disease at all health-care } \\
\text { facilities (including community facilities and } \\
\text { pharmacies) } \\
\text { Access to home urine glucose sticks for patients to } \\
\text { assess glycosuria at least three times per week } \\
\text { Availability of affordable and sustainable technologies } \\
\text { for self-monitoring of blood glucose in those people } \\
\text { with diabetes using insulin } \\
\text { Availability of affordable or portable digital photography } \\
\text { equipment for taking and transmitting retinal images } \\
\text { for remote interpretation } \\
\text { Availability of affordable or portable equipment for } \\
\text { retinal image acquisition, image transfer, and image } \\
\text { grading } \\
\text { Provision of laser equipment for treatment of diabetic } \\
\text { retinopathy }\end{array}$ & $\begin{array}{l}\text { Availability of affordable and sustainable self- } \\
\text { monitoring of blood glucose equipment for all patients } \\
\text { with diabetes, and availability of glucose strips for } \\
\text { testing at least three times per day on } 3 \text { days per week } \\
\text { Therapeutic options for erectile dysfunction } \\
\text { Orthotics for people with foot deformities to prevent } \\
\text { ulceration } \\
\text { Availability of intravitreal anti-VEGF drugs to treat } \\
\text { diabetic retinopathy }\end{array}$ \\
\hline Service delivery & $\begin{array}{l}\text { Availability of services to provide education and } \\
\text { counselling for patients with diabetes or gestational } \\
\text { diabetes at all health-care facilities } \\
\text { Availability of screening for gestational diabetes with } \\
2013 \text { WHO criteria3 for high-risk women attending } \\
\text { antenatal services at all health-care facilities } \\
\text { Availability of regular (3 monthly) follow-up for diabetes } \\
\text { and associated conditions via community health } \\
\text { workers or primary care facilities } \\
\text { Availability of services to deliver regular supplies of } \\
\text { medicines within patients' local areas } \\
\text { Clearly delineated referral pathways between } \\
\text { community, secondary, and specialised or tertiary care } \\
\text { centres }\end{array}$ & $\begin{array}{l}\text { Availability of laboratory tests for fasting blood glucose, } \\
\mathrm{HbA}_{1 c} \text { and renal function at all secondary and tertiary } \\
\text { referral centres } \\
\text { Availability of services to screen for diabetic retinopathy, } \\
\text { integrated into general services for diabetes care } \\
\text { Access to tertiary care facilities for treatment of cataracts } \\
\text { and retinopathy } \\
\text { Availability of community support groups } \\
\text { Availability of annual review at a specialist diabetes clinic } \\
\text { (with facilities for annual } \mathrm{HbA}_{1 \mathrm{c}} \text { measurement, screening } \\
\text { for complications, assessment of proteinuria with urine } \\
\text { strips, measurement of serum creatinine, retinal } \\
\text { photography for people at high risk of retinopathy, and } \\
\text { monofilament or tuning fork testing for peripheral } \\
\text { neuropathy) for all patients with diabetes }\end{array}$ & $\begin{array}{l}\text { Access to secondary or tertiary care facilities for cataract } \\
\text { surgery and treatment of diabetic retinopathy with laser } \\
\text { and intravitreal anti-VEGF drugs } \\
\text { Access to tertiary care facilities for vitreoretinal surgery } \\
\text { to treat advanced diabetic retinopathy } \\
\text { Wide-scale access to dialysis and renal transplantation, } \\
\text { vascular surgery for peripheral vascular disease, and } \\
\text { percutaneous coronary intervention and coronary artery } \\
\text { bypass grafting at tertiary care facilities } \\
\text { Rehabilitation programmes and prostheses for people } \\
\text { after a stroke or amputation }\end{array}$ \\
\hline Information & $\begin{array}{l}\text { At the diabetes clinic level, ensure availability of simple, } \\
\text { future-proof diabetes registries to allow documentation of } \\
\text { diabetes control, medications, and complications, and } \\
\text { triggering of recall } \\
\text { Availability of information technology to allow remote } \\
\text { interpretation of digital retinal photos taken in clinics in } \\
\text { which no expertise in interpretation is available } \\
\text { Availability of basic diabetes education, including } \\
\text { information about prevention and detection of } \\
\text { complications, that is culturally appropriate, provided in } \\
\text { a local language, and understandable to patients and } \\
\text { their relatives } \\
\text { Widespread availability of information about and } \\
\text { campaigns to promote a healthy lifestyle that is tailored } \\
\text { to the local population, culturally appropriate, and } \\
\text { delivered through media platforms that are widely used } \\
\text { by the local population } \\
\text { Availability of a basic cardiovascular risk calculator at } \\
\text { each primary health-care facility }\end{array}$ & $\begin{array}{l}\text { Availability of electronic links between specialist } \\
\text { diabetes clinics and primary care providers, allowing } \\
\text { updates on test results, prescription modification, and } \\
\text { follow-up and referral information } \\
\text { Availability of a cloud-based storage system for storage } \\
\text { of and access to electronic diabetes records by primary } \\
\text { and secondary providers } \\
\text { Availability of a mobile phone alert system to } \\
\text { communicate results and changes to treatments to } \\
\text { patients and to alert patients to follow-up appointments } \\
\text { Availability of equipment allowing yearly training } \\
\text { updates for primary health-care workers and others } \\
\text { providing diabetes care, to be delivered by distance } \\
\text { learning }\end{array}$ & $\begin{array}{l}\text { Fully integrated, cloud-based patient information } \\
\text { systems for storing diabetes-relevant information along } \\
\text { with all other key records of diagnoses and treatment }\end{array}$ \\
\hline
\end{tabular}




\begin{tabular}{|c|c|c|c|}
\hline & Level one & Level two & Level three \\
\hline \multicolumn{4}{|c|}{ (Continued from previous page) } \\
\hline Workforce & $\begin{array}{l}\text { Training (with ongoing regular support and mentoring) } \\
\text { of enough community health-care workers (appropriate } \\
\text { to current and projected burden) in each region to aid } \\
\text { case finding and provide adherence support and } \\
\text { preventive care and information } \\
\text { Training of health-care workers who provide antenatal } \\
\text { care to recognise women at high risk of gestational } \\
\text { diabetes and refer them to antenatal services offering } \\
\text { screening and treatment } \\
\text { Ensure all primary care providers are trained to recognise } \\
\text { and treat hyperglycaemia } \\
\text { Ensure all primary care providers are trained to screen for } \\
\text { and treat associated cardiovascular risk factors in } \\
\text { patients with diabetes and recognise when to refer a } \\
\text { patient for more specialised care } \\
\text { Provision of training on recognition and referral of } \\
\text { diabetic retinopathy cases to the existing eye-care } \\
\text { workforce }\end{array}$ & $\begin{array}{l}\text { Trained diabetes educators in each district to support } \\
\text { community health workers and people with diabetes } \\
\text { Training for all health-care providers to recognise the } \\
\text { symptoms of diabetes and its complications, as well as the } \\
\text { means of diagnosis } \\
\text { Ensure availability of one paediatric and one adult } \\
\text { endocrinologist at each tertiary care centre in each } \\
\text { country } \\
\text { Provide training on diabetes to traditional healers to } \\
\text { allow them to recognise the symptoms and refer } \\
\text { potential sufferers to medical care } \\
\text { Ensure availability of appropriately trained retinal } \\
\text { photographers and retinal image graders } \\
\text { Ensure availability of one or more ophthalmologist } \\
\text { trained to deliver treatment for diabetic retinopathy in } \\
\text { each tertiary care centre in each country }\end{array}$ & $\begin{array}{l}\text { Specialist diabetes workforce expanded to enable one } \\
\text { annual visit for each patient with a fully trained } \\
\text { diabetologist } \\
\text { Availability of multidisciplinary teams for the } \\
\text { management of hyperglycaemia in pregnancy }\end{array}$ \\
\hline Financing & $\begin{array}{l}\text { Ensure all necessary care for diabetes and associated } \\
\text { cardiovascular risk factors is made freely available to } \\
\text { those living below the World Bank } 2015^{4} \text { defined poverty } \\
\text { line of US } \$ 1.90 \text { per day }\end{array}$ & $\begin{array}{l}\text { Ensure all essential care for diabetes and associated } \\
\text { cardiovascular risk factors (including access to screening, } \\
\text { regular follow-up [including blood tests], access to } \\
\text { medicines for primary and secondary prevention, } \\
\text { follow-up by a specialist once per year, and detection of } \\
\text { microvascular complications) is covered by universal } \\
\text { health coverage schemes such that no patient has to pay } \\
\text { direct costs of care out of pocket }\end{array}$ & $\begin{array}{l}\text { Ensure availability of detection and treatment facilities } \\
\text { for macrovascular and microvascular complications of } \\
\text { diabetes, with no patient suffering catastrophic } \\
\text { expenditure for accessing care }\end{array}$ \\
\hline Knowledge & $\begin{array}{l}\text { Ascertain most appropriate method (in terms of } \\
\text { predicting future complications) for diagnosing diabetes } \\
\text { in sub-Saharan Africa } \\
\text { Ascertain most appropriate method (in terms of ability } \\
\text { to predict maternal and neonatal complications) of } \\
\text { diagnosing gestational diabetes } \\
\text { Ascertain most appropriate method for retinal image } \\
\text { acquisition, image grading, and targeted case detection } \\
\text { in low-resource environments }\end{array}$ & $\begin{array}{l}\text { Obtain reliable in-country estimates (for example, with } \\
\text { the STEPwise approach to surveillance method }{ }^{5} \text { ) from } \\
\text { adequately sized samples of the burdens of obesity, } \\
\text { diabetes, and other cardiovascular risk factors } \\
\text { Obtain reliable in-country estimates from adequately } \\
\text { sized samples of the burden of gestational diabetes } \\
\text { Assess ability of country health systems to provide care } \\
\text { for in-country burden of patients with diabetes }\end{array}$ & $\begin{array}{l}\text { Development of a sub-Saharan African NCD research } \\
\text { group and plan that includes a strategy for collaboration } \\
\text { and data sharing } \\
\text { Ensure that research of diabetes and other NCDs in sub- } \\
\text { Saharan Africa (including clinical trials) is led by } \\
\text { researchers residing in sub-Saharan Africa }\end{array}$ \\
\hline $\begin{array}{l}\text { Leadership and } \\
\text { governance }\end{array}$ & $\begin{array}{l}\text { Maintain a dedicated team within the Ministry of Health } \\
\text { with responsibility for overseeing country-wide, phased } \\
\text { rollout of diabetes care } \\
\text { Appoint a dedicated government minister with } \\
\text { responsibility for developing and overseeing } \\
\text { government policies to prevent obesity and overweight } \\
\text { and encourage healthy lifestyles } \\
\text { Maintain a dedicated team within the Ministry of Health } \\
\text { with responsibility for ensuring wide-scale rollout of } \\
\text { public health messages and policies to prevent and } \\
\text { reverse obesity and overweight } \\
\text { WHO to assemble working groups (consisting of } \\
\text { practitioners, health policy makers, laboratory and } \\
\text { diagnostic experts, device manufacturers, and health } \\
\text { information technology companies) to produce a list of } \\
\text { essential diagnostics and diagnostic equipment for NCDs } \\
\text { and to create guidelines on technical standards }\end{array}$ & $\begin{array}{l}\text { Integrate teams within ministries that deal with diabetes } \\
\text { care and prevention into wider NCD teams } \\
\text { Introduce taxation policies for sugar-sweetened } \\
\text { beverages and policies to ensure all new urban } \\
\text { developments are walkable } \\
\text { Integration of members of teams within ministries that } \\
\text { deal with diabetes care and prevention into teams } \\
\text { addressing universal health coverage strategies } \\
\text { WHO to assemble a working group to advise on } \\
\text { information technology solutions for integrated patient } \\
\text { records, which include diabetes and other NCD registries, } \\
\text { and to explore future-proof and interoperable cloud- } \\
\text { based solutions that can be used in LMICs }\end{array}$ & $\cdot$ \\
\hline
\end{tabular}

as part of a rigorous longitudinal outcomes study that also compares different tests for diagnosis of hyperglycaemia.

\section{Introduction}

Sub-Saharan Africa is experiencing a rapid increase in the prevalence of non-communicable diseases (NCDs). Although, in 2015, Africa was the only continent in the world where morbidity and mortality from infectious diseases still surpassed that from NCDs, this balance will soon change as the region experiences the full impact of the increase in NCDs. ${ }^{6}$

Rapid demographic (growing and ageing population), sociocultural (lifestyle changes and eating habits), and economic (higher income, urbanisation, changing food availability, and evolving lifestyle and work practices) transitions $\mathrm{s}^{7,8}$ are driving increases in the risk factors and
Centre for Modern Indian Studies \& Department of Economics, Goettingen, Germany (CBommer MA, E Heesemann MSc, V Sagalova MA, SVollmer); University of Liverpool, Liverpool, UK (PI Burgess FRCOphth PhD); 
International Centre for Eye Health, Faculty of Infectious \& Tropical Diseases (M) Burton PhD), and Department of NCD Epidemiology (M) Nyirenda PhD), London School of Hygiene and Tropical Medicine, London, UK; Malawi Epidemiology and Intervention Research Unit, Lilongwe, Malawi (F P Chilunga); The Fletcher School of Law and Diplomacy, Tufts University, Medford, MA, USA (A Conn MA); Department of Paediatrics and Adolescent Health, Faculty of Medicine, University of Botswana and Princess Marina Hospital,

Gaborone, Botswana (D R Joel MRCPI); The Dartmouth Institute for Health Policy and Clinical Practice, Dartmouth College, Hanover, NH, USA (A W Eagan MSW); Rwanda Diabetes Association, Kigali, Rwanda (C Gishoma); Leiden

University, Science Based Business, Leiden, Netherlands (S Jong PhD); NonCommunicable Diseases Research Unit, South African Medical Research Council, Cape Town, South Africa (A P Kengne PhD); Joslin USA (Y Khan MD); University of Texas Southwestern Medical Center, Dallas, TX, USA (R Kharel); Kenya Ministry of Health, Division of

Non-communicable diseases, Nairobi, Kenya (KJ Mwangi MSc); Division of Diabetic Medicine \& Endocrinology (N S Levitt MD FCP), and Chronic Department of Medicine (N S Levitt, M Werfalli PhD), Diabetes Center, Boston, MA, Disease Initiative for Africa,

prevalence of diabetes and other risk factors of cardiovascular disease. In sub-Saharan Africa, between 1980 and 2015, the number of people older than 20 years with a BMI of greater than $25 \mathrm{~kg} / \mathrm{m}^{2}$ increased from 28 million to 127 million, while the disease burden attributed to diabetes, as measured by disability-adjusted life-years (DALYs), increased by $88 \% .^{9}$ More than $90 \%$ of diabetes cases in sub-Saharan Africa are type 2 diabetes, suggesting that modifiable risk factors are major contributors to the burden of disease. ${ }^{10}$

Against this backdrop of dramatic statistics is the uncomfortable reality that most estimates for diabetes, other cardiovascular disease risk factors, and cardiovascular disease burden per se in countries in subSaharan Africa have been modelled from generally poor-quality evidence using methods of defining diabetes that have been developed in high-income countries (HICs), often with European-origin populations. In the case of diabetes, a 2016 study $^{11}$ found that nationally representative empirical biomarker data on diabetes diagnosis were not available from 21 of countries in subSaharan Africa. So, although there is reasonable evidence that diabetes and other cardiovascular risk factors are increasing, the certainty of the situation is far from clear. Most (34 [71\%] of 48 countries) of the world's leastdeveloped countries are in sub-Saharan Africa. ${ }^{12}$ Thus, although the focus of development aid and research funding has so far been on infectious diseases, ${ }^{13,14}$ many countries are still struggling to develop health systems to cope with the existing infectious disease burden, which is much better delineated than that of cardiovascular disease and its risk factors. It therefore seems an impossible task to ask these countries - as has been requested by the Sustainable Development Goals (SDGs) and the UN high-level meeting on $\mathrm{NCDs}^{15}$ - to respond to an as yet not accurately quantified burden of cardiovascular disease risk factors, using methods not yet proven to be effective in the region, and with little funding.

Driven by this recognition, a group of academics, policy makers, and clinicians met at the Rockefeller Foundation

\begin{tabular}{|c|c|c|c|c|c|c|}
\hline & \multicolumn{2}{|c|}{ Prevalence (\%) } & \multirow[t]{2}{*}{$\begin{array}{l}\text { Percentage change } \\
\text { for } 1980-2014\end{array}$} & \multicolumn{2}{|c|}{$\begin{array}{l}\text { Number } \\
\text { (millions) }\end{array}$} & \multirow[t]{2}{*}{$\begin{array}{l}\text { Percentage change } \\
\text { for } 1980-2014\end{array}$} \\
\hline & 1980 & 2014 & & 1980 & 2014 & \\
\hline African region & $3 \cdot 1 \%$ & $7 \cdot 1 \%$ & $129 \cdot 0 \%$ & 4 & 25 & $525 \cdot 0 \%$ \\
\hline Region of the Americas & $5 \cdot 0 \%$ & $8 \cdot 3 \%$ & $66 \cdot 0 \%$ & 18 & 62 & $244 \cdot 4 \%$ \\
\hline $\begin{array}{l}\text { Eastern Mediterranean } \\
\text { region }\end{array}$ & $5.9 \%$ & $13 \cdot 7 \%$ & $132 \cdot 2 \%$ & 6 & 43 & $616 \cdot 7 \%$ \\
\hline European region & $5 \cdot 3 \%$ & $7 \cdot 3 \%$ & $37 \cdot 7 \%$ & 33 & 64 & $93 \cdot 9 \%$ \\
\hline Southeast Asia region & $4 \cdot 1 \%$ & $8.6 \%$ & $109 \cdot 8 \%$ & 17 & 96 & $464 \cdot 7 \%$ \\
\hline Western Pacific region & $4 \cdot 4 \%$ & $8.4 \%$ & $90 \cdot 9 \%$ & 29 & 131 & $351 \cdot 7 \%$ \\
\hline Worldwide total & $4 \cdot 7 \%$ & $8 \cdot 5 \%$ & $80 \cdot 9 \%$ & 108 & 422 & $290 \cdot 7 \%$ \\
\hline
\end{tabular}

Table 2: Estimated prevalence of diabetes and number of adults aged 18 years or older with diabetes in 1980 and 2014, by WHO region
Bellagio Center in Italy in March, 2015, to discuss the state of play and potential routes forward. We centred our discussions on diabetes-a preventable cardiovascular disease risk factor-because of its multi-system interactions and adverse outcomes and because broad improvements in health-care systems are needed for its management. We formed The Lancet Diabetes \& Endocrinology Commission on Diabetes in sub-Saharan Africa. After the meeting in Bellagio, we organised ourselves into working groups to look at both clinical and health-system elements involved in diabetes and diabetes care in sub-Saharan Africa. We met again at the World Diabetes Congress in Vancouver, Canada, in November, 2015, to discuss progress. The final Commission meeting was held at the Harvard Medical School Center for Global Health Delivery, Dubai, in February, 2016, to report results, discuss areas in which we had found equipoise, agree on the best approaches to advise in these areas, and structure the report.

Here, we present the findings of the Commission, including discussions of burden, and clinical, economic, and broader health systems, to inform the development of an effective response to the increasing burden of diabetes, other cardiovascular disease risk factors, and their adverse consequences. The methods used in sections of the report that involved novel research can be found in detail in the appendix 1 . The narrative sections of the report were informed by extensive reviews of the literature and the knowledge and opinions of the Commissioners.

\section{Burden of diabetes in sub-Saharan Africa}

Globally, according to the NCD Risk Factor Collaboration (NCD-RisC), the number of adults with diabetes increased from 108 million (a prevalence of 4.7\%) in 1980 to 422 million $(8 \cdot 5 \%)$ in 2014 an increase in prevalence of $80.9 \% .{ }^{16}$ Notably, diabetes prevalence in the WHO African region increased by $129.0 \%$ (from $3 \cdot 1 \%$ in 1980 to $7 \cdot 1 \%$ in 2014 ; table 2 ). This increase is second only to the WHO eastern Mediterranean region, in which the prevalence of diabetes increased by $132 \cdot 2 \%$ (from $5 \cdot 9 \%$ in 1980 to $13 \cdot 7 \%$ in 2014 ; table 2 ).

Unfortunately, the various estimates of diabetes prevalence in sub-Saharan Africa have used different methodologies, all of which have limitations (panel 2). Hence, no clear data exist on the prevalence of diabetes in this region, which is important for planning an adequate health-systems response. In addition to WHO and NCD-RisC, other well cited estimates come from the International Diabetes Federation (IDF) ${ }^{25}$ and the Global Burden of Disease (GBD) group at the Institute for Health Metrics and Evaluation, ${ }^{9}$ which has produced estimates of deaths and DALYs due to diabetes. However, despite differences in methodologies, all estimates point to an increasing burden of diabetes in sub-Saharan Africa. Notwithstanding the limitations of the methods used to estimate diabetes prevalence (panel 2), the 
estimates used in this report are from NCD-RisC, ${ }^{16}$ unless otherwise stated. We have chosen these estimates because they have been adopted by WHO and are therefore likely to gain global credibility.

The increasing impact of diabetes in sub-Saharan Africa is reflected in results from the 2015 GBD study, ${ }^{9}$ which estimated that diabetes in the region caused an average of 145189 deaths and 5556560 DALYs; DALYs attributable to diabetes increased by $88 \%$ between 1990 and 2010. Inevitably, there is considerable imprecision in such estimates and other groups have produced different figures. For example, the IDF estimated that, in 2015, around 321000 deaths in sub-Saharan Africa were attributable to diabetes, and that $79 \%$ of those deaths occurred in people aged 60 years or younger-a proportion higher than in any other region in the world. ${ }^{25}$

\section{Type 2 diabetes in sub-Saharan Africa Overview}

As in other parts of the world, more than $90 \%$ of people living with diabetes in sub-Saharan Africa have type 2 diabetes, ${ }^{10,26}$ which is typically associated with increasing age and overweight or obesity. We refer to type 2 diabetes simply as diabetes throughout this report, and specify type when referring to other types of diabetes.

\section{Drivers of type 2 diabetes}

Overweight and obesity

Traditionally, lifestyle factors leading to overweight and obesity, and then to diabetes, have been considered modifiable. However, it is now increasingly acknowledged that, at least at the individual level, lifestyle drivers of diabetes are difficult to modify; individuals have far less agency than was previously thought. ${ }^{8,27}$ Additionally, genetic predisposition to overweight, obesity, and diabetes-once thought to be relatively stable over geological timeframes-is now known to be more modifiable in the short term than previously considered, possibly via intergenerational epigenetic changes (see panel 3 for a discussion of genetic drivers of diabetes in sub-Saharan Africa). Although susceptibility to diabetes and its risk factors varies, the condition is largely avoidable with appropriate interventions.

Although not all people with diabetes in sub-Saharan Africa are overweight or obese by western standards, a rapid increase in the prevalence of overweight and obesity is undoubtedly a major driver of the increasing prevalence of type 2 diabetes in the region. ${ }^{9,16}$ The contribution of overweight and obesity to the variance of the prevalence of diabetes in sub-Saharan Africa is an as of yet unanswered question; some studies ${ }^{37-39}$ suggest that African populations might develop diabetes at lower body mass than people of European origin, and other studies in different settings ${ }^{40,41}$ have suggested that, for the same level of obesity, African populations are at lower risk for diabetes than their western counterparts. However, high-quality, convincing evidence is scarce.
Globally, overweight and obesity are traditionally considered conditions of urban rather than rural populations, although this situation is now in transition. In HICs, overweight and obesity are seen more in rural populations than in urban populations..$^{21,41}$ Few high-quality longitudinal studies have been done in Africa, but those that have been done suggest that the prevalence of overweight and obesity has increased

University of Cape Town, Cape Town, South Africa; Yale-New Haven Hospital, New Haven, CT, USA (C P Malm MD); Aga Khan University Hospital, Nairobi, Kenya (FW Manguyu MMed); Beth Israel Deaconess Medical Center, Boston, MA, USA (J Manne-Goehler); The

Panel 2: Estimating the prevalence of diabetes in sub-Saharan Africa

Several different estimates exist for the prevalence of diabetes in countries in the sub-Saharan African region. These estimates vary depending on the method used to ascertain the diagnosis (eg, self-report vs biomarker) and, for biomarker-based diagnosis only, the biomarker used (eg, fasting glucose vs $2 \mathrm{~h}$ oral glucose tolerance test [OGTT] vs $\mathrm{HbA}_{1 \mathrm{c}}$ ). Even in well studied populations in high-income countries (HICs), debate continues around which biomarker most reliably predicts long-term macrovascular and microvascular outcomes of hyperglycaemia and, hence, which should be used to diagnose diabetes. ${ }^{1718}$ No longitudinal studies have been done in sub-Saharan Africa to ascertain the best method for diagnosis. Thus, whether methods developed in HICs to determine the prevalence of diabetes can be used to define diabetes and reliably predict the future burden of clinically relevant outcomes in populations in sub-Saharan Africa is currently unknown. Studies in this area are urgently needed.

Compounding this uncertainty is that many countries have used the WHO STEPwise approach to surveillance (STEPS) method to estimate burden, ${ }^{5}$ which has included use of fasting glucose to diagnose diabetes. This inclusion could potentially miss patients in the earlier stages of the disease who would show impaired glucose tolerance in a $2 \mathrm{~h}$ OGTT but would have normal fasting concentrations. The effects of these differences in methods on cross-sectional prevalence estimates of diabetes have been shown in African and other settings. ${ }^{19}$ For example, the prevalence of diabetes in people aged 55 years and older in Africa was higher when measured with the $2 \mathrm{~h}$ OGTT than when measured with fasting glucose alone (23.9\% vs $10.9 \%)$ and in non-STEPS studies than in STEPS studies (17.1\% vs $9 \cdot 6 \%)^{20}$

Results can also be affected by the sampling method used, with many country estimates coming from samples of convenience rather than from robust methods of sampling. Sample sizes also vary greatly between studies-for example, in the NCD-RisC study, ${ }_{1}^{16}$ some countries had less than 150 data points and others had tens of thousands. Geography and demographics should also be considered when interpreting results. For example, major differences exist in the prevalence of obesity and diabetes between populations of young and older people. ${ }^{20}$ Additionally, although high-quality data on the urban versus rural prevalence of diabetes and obesity are scarce, studies from other countries clearly show differences in prevalence between rural and urban areas and a change in that distribution over time. ${ }^{21}$ Detection bias should also be considered, because underdeveloped health systems in countries in sub-Saharan Africa might lead to under-reporting of diabetes prevalence compared with $\mathrm{HICs}$, where diabetes is more reliably detected.

These factors need to be considered when interpreting all studies of diabetes prevalence in countries in sub-Saharan Africa. Additionally, and importantly, all studies producing estimates for the region as a whole-and individual countries within that regionare modelled and often based on little or no data. Even though we have chosen to use estimates from NCD-RisC ${ }^{16}$ for illustration, and recognise that this work has utility in making comparisons across regions and over time, the reliability of the prevalence data for individual countries in sub-Saharan Africa is questionable. ${ }^{22-24} 21$ countries in sub-Saharan Africa had missing data and, in those countries with data, samples were often small and data were old. 
Panel 3: Genetic drivers of diabetes

\section{Genetics of type 2 diabetes in sub-Saharan Africa}

We are not aware of any initiative that has successfully integrated genetic markers into clinically usable risk prediction scores (especially in low-income and middle-income countries) or that has used genetics to successfully improve treatment outcomes in diabetes. Nevertheless, genetics continues to be an area of interest both to funders and researchers. We question the need for such research investment in a geographical area in which even the burden of disease is unknown. However, given the interest in the area, and the possibility that knowledge of genetic susceptibility to diabetes might help define the scale and direction of the diabetes epidemic in Africa, we present a brief overview of the field in sub-Saharan Africa.

In global populations, nearly 80 genetic loci have been implicated in susceptibility to type 2 diabetes, ${ }^{28,29}$ and about $50 \%$ of these risk loci were replicated in a 2015 study of three sub-Saharan African countries enrolled in the AADM study.$^{30}$ The results of that study suggested that the genetic architecture of type 2 diabetes in sub-Saharan Africa is probably characterised by several risk loci shared with populations of non-African ancestry, and that genetic data from Africans promise to inform the genetics of all human populations. Epigenetic changes have also been shown to have differential effects on diabetes incidence depending on the population studied, and such changes might be very important in African populations given early-life risks of undernutrition. ${ }^{31}$ Studies ${ }^{30,31}$ in sub-Saharan African populations suggest that natural selection has acted on several genomic regions associated with obesity and type 2 diabetes, and a study ${ }^{32}$ that mapped the genetic risk of type 2 diabetes by measuring the allelic frequency of 16 diabetes-associated variants in 51 populations suggested that Africans face the greatest known genetic risk for type 2 diabetes of any ethnicity studied thus far.

Some knowledge gaps can also be filled by the study of African American populations, although such comparisons must be interpreted with caution because African Americans mostly originated from west or central Africa and are therefore not representative of sub-Saharan Africa as a whole..$^{33}$ Nonetheless, African American populations clearly have a two-times increase in the risk of type 2 diabetes compared with Americans of European origin, ${ }^{34,35}$ and they also have a much higher prevalence of type 2 diabetes than most African populations. ${ }^{16}$ This increased risk of diabetes in African Americans might be an indication of future trends in Africa. Numerous initiatives linking genetics and cardiovascular risk are underway in sub-Saharan Africa, including the H3Africa and RODAM studies.

\section{Genetics of type 1 diabetes in sub-Saharan Africa}

Little is known about the genetics of type 1 diabetes in sub-Saharan Africa. However, a study ${ }^{36}$ of African American populations showed large diversity of

HLA DRB1-DQA1-DQB1 haplotypes and genotypes in African, compared with

European, descendants. Association analyses reproduced several type 1 diabetes risk effects seen in European-derived haplotypes, while also showing novel effects for African-derived haplotypes. In particular, the African-specific DR3 haplotype DRB1*0 3:02-DQA1 ${ }^{*} 04: 01-D Q B 1{ }^{*} 04: 02$ was protective against type 1 diabetes. ${ }^{36}$ Additionally, the DR4/DR9 genotype, which contains an African-derived DR9 haplotype, conferred an odds ratio of 30.88 compared with the highest-risk genotypes found in populations of European origin. ${ }^{36}$

Institute of HIV/AIDS, Disease Prevention \& Control, Rwanda

Biomedical Center, Kigali,

Rwanda (M A Muhimpundu, SP Niyonsenga, S Rwunganira); Ministry of Health,

Government of Malawi, Lilongwe, Malawi

(B M Mwagomba); Moi in urban areas. For example, Ziraba and colleagues ${ }^{42}$ used data from Demographic and Health Survey studies done between 1992 and 2005 in seven sub-Saharan African countries to show that overweight and obesity had increased by $35 \%$ in women living in urban areas, and that $31.4 \%$ of urban women were overweight or obese in 2005, ranging from $28-29 \%$ in Burkina Faso and Senegal to $38 \%$ in Kenya. However, it seems that the picture in sub-Saharan Africa is mixed: some countries show a greater prevalence of overweight and obesity among urban dwellers, whereas some show a greater prevalence in rural districts. ${ }^{43}$ This mixed picture is probably explained in part by differences in the pace of the ongoing rural transformation across Africa. ${ }^{44}$ Figure 1 shows our analysis of the interactions between diabetes and obesity in countries in subSaharan Africa.

Overweight and obesity are generally associated with low socioeconomic status in HICs and high socioeconomic status in low-income and middle-income countries (LMICs), known as the reversal hypothesis. ${ }^{45}$ However, substantial variation is seen at a more granular level. For example, in sub-Saharan Africa, Ziraba and colleagues $^{42}$ found that overweight and obesity were more prevalent in women of low socioeconomic status than in women of high socioeconomic status, with the most rapid increase in prevalence (50\% overall) seen in the poorest people living in urban areas; the prevalence of overweight and obesity declined by $10 \%$ in women with secondary education or higher. Conversely, AitsiSelmi and colleagues ${ }^{46}$ found a positive association between education and obesity in two sub-Saharan African countries (Nigeria and Benin), and a small crosssectional study ${ }^{47}$ in rural Uganda suggested that obesity was a greater problem among those of high socioeconomic status than among those of low socioeconomic status. Manne-Goehler and colleagues ${ }^{11}$ found a strong education gradient in the self-awareness of overweight and obesity, suggesting that, in the long term, education and high socioeconomic status might be associated with reduced prevalence. Variation in results might also depend on the definitions of socioeconomic status used in different studies.

Such heterogeneity in results comparing urban with rural areas and high with low socioeconomic status is expected in a rapidly changing environment, but the paucity of high-quality studies examining these issues restricts any clear conclusions about links between place of residence, socioeconomic status, and drivers of obesity. The scarcity of reliable information also limits the ability to suggest appropriate interventions.

Poor nutrition during fetal and early life, which is known to contribute to obesity-related health problems in later life, especially when combined with subsequent abundance of food (the so-called thrifty phenotype ${ }^{48}$ ), is a likely problem in sub-Saharan Africa. ${ }^{40}$ Some high-quality evidence for this phenomenon is beginning to emerge from the region. For example, a study ${ }^{49}$ of 352 Malawian children (median age 9.3 years) who had been treated for severe acute malnutrition at an average age of 24 months showed them to have clear evidence of thrifty growth compared with sibling and community controls, despite evidence of catch-up in growth. Further research is needed to assess the long-term impact of intrauterine and childhood malnutrition in Africa. 
Cultural factors also affect the prevalence of overweight and obesity - for example, a large girth is perceived as a sign of affluence in many countries in sub-Saharan Africa and is a deeply rooted status symbol conferring influence, health, and attractiveness. ${ }^{50}$ Excess weight also has positive connotations in societies in which strong stigma is attached to weight loss and wasting associated with HIV/AIDS. ${ }^{10}$
Teaching and Referral Hospital, Uasin Gishu, Kenya (B Njuguna B Pharm); NCD Theme, MRC/UVRI Uganda Research Unit, Entebbe, Uganda

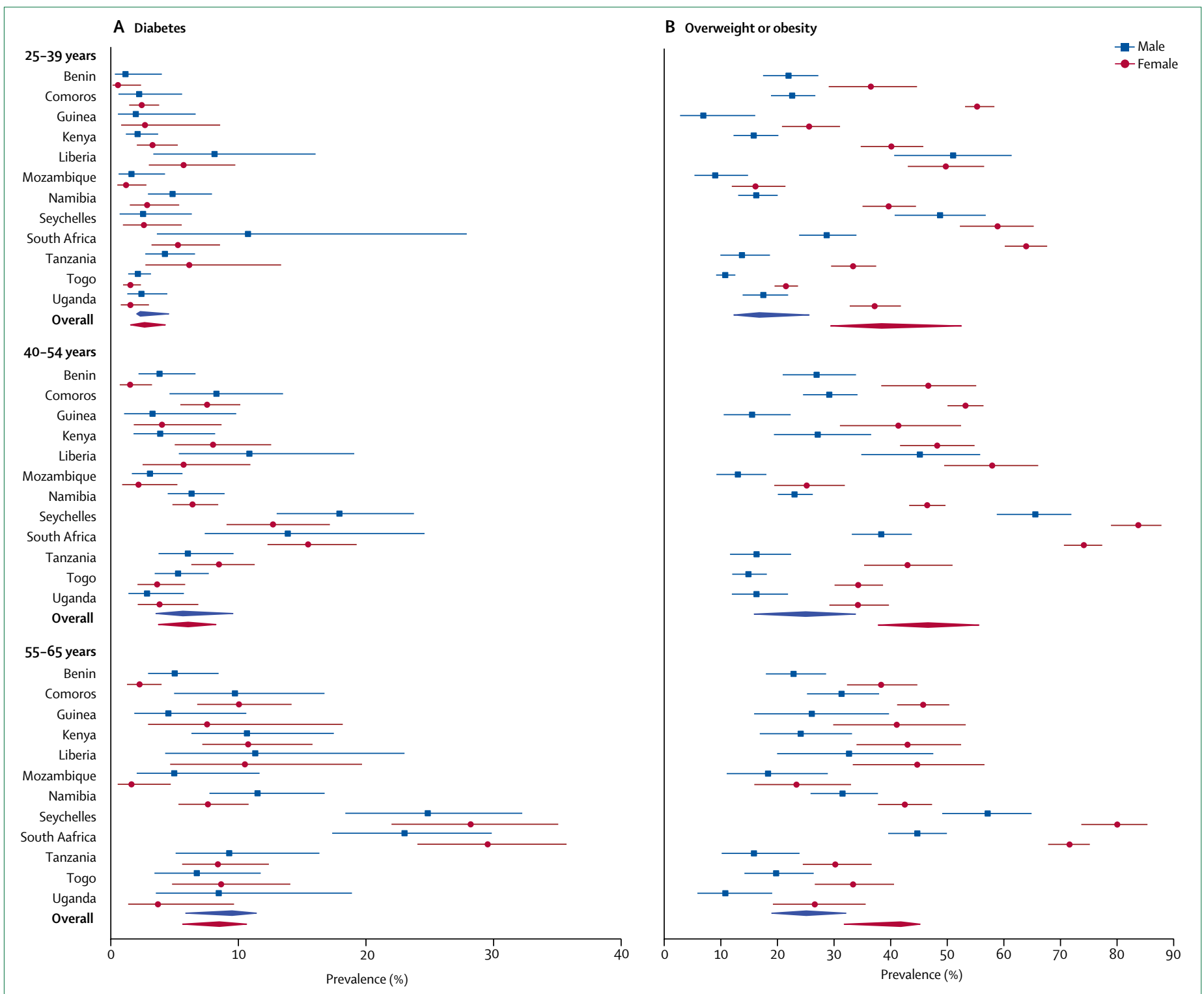

Figure 1: Age-stratified and sex-stratified prevalence of diabetes and overweight or obesity among adults in 12 countries of sub-Saharan Africa

Reproduced from Manne-Goehler and colleagues, with permission from Elsevier. Each point shows the point prevalence estimate for each age group in each country and the horizontal bars indicate $95 \% \mathrm{Cls}$, except for the overall prevalence for each age group, which is also represented but as median and IQR. The data sources and methods are discussed in detail elsewhere ${ }^{11}$ and a summary is included in the appendix 1. Efforts to obtain, from WHO, data from the STEPwise Approach to Surveillance (STEPS) surveys done across Africa were unsuccessful. ${ }^{24}$ We therefore independently approached each country directly. The complete pooled dataset included 39062 individuals from 12 countries in the period of 2004-13. (A) We calculated the prevalence of diabetes by age and sex. The surveys included WHO STEPS surveys done in Benin (2008), Comoros (2011), Guinea (2007-08), Liberia (2011), Mozambique (2005), Tanzania (2012), Togo (2010), and Seychelles (2013); a Demographic and Health Survey done in Namibia in 2013; and the South Africa Nutrition and Health Examination Survey done in 2012 (appendix 1). We defined diabetes according to the WHO criteria (appendix 1) as either a fasting plasma glucose concentration of $7.0 \mathrm{mmol} / \mathrm{L}\left(126 \mathrm{mg} / \mathrm{dL}\right.$ ) or higher, a $2 \mathrm{~h}$ plasma glucose concentration of $11.1 \mathrm{mmol} / \mathrm{L}\left(200 \mathrm{mg} / \mathrm{dL}\right.$ ) or higher, or an HbA $\mathrm{A}_{1 \mathrm{c}}$ measurement of $6.5 \%$ or higher. Crosscountry prevalence of diabetes ranged from $1.3 \%$ in Benin to $21.6 \%$ in Seychelles, based on fasting blood glucose measurement and self-reported use of diabetes medications. The analysis showed a strong association between increasing age and the prevalence of diabetes, with the prevalence reaching around 30\% in men and women aged $55-64$ years in South Africa and Seychelles. A higher prevalence in women than in men was seen across all age groups. (B) We calculated the prevalence of overweight or obesity by age and sex. Overweight or obesity was defined as a BMI of $25 \mathrm{~kg} / \mathrm{m}^{2}$ or higher. The analysis showed a strong association between increasing age and the prevalence of overweight or obesity, with a higher prevalence in women than in men across all age groups. Prevalence of overweight or obesity also varied across countries, with age-stratified levels ranging from less than $10 \%$ in men aged $25-39$ years in Benin and Togo, to more than $70 \%$ in South Africa, and to almost $80 \%$ in women aged $55-65$ years in Seychelles. 
(M) Nyirenda); International Diabetes Federation Life for a Child Program, Glebe, NSW, Australia (G D Ogle FRACP); Diabetes NSW \& ACT, Glebe, NSW, Australia (G D Ogle); Partners In Health, Rwinkwavu, South Kayonza, Rwanda (P H Park MD); Purdue University College of Pharmacy (Purdue Kenya Partnership), Indiana Institute for Global Health, Uasin Gishu, Kenya

(S D Pastakia PhD); The Ohio State University, Columbus, $\mathrm{OH}$, USA (C Pekny PharmD); Shree Hindu Mandal Hospital, Dar Es Salaam, Tanzania

(K Ramaiya MBBS MMed); Department of Internal Medicine, Addis Ababa University, Addis Ababa,

Ethiopia (A Reja); Center for

Research on Genomics and Global Health, National Institutes of Health, Bethesda, MD, USA (C N Rotimi PhD); University of Vermont, Burlington, VT, USA (G Sarriera); Department of Medicine, and

Clinical Epidemiology Unit, Makerere University College of Health Sciences, Kampala, Uganda (N K Sewankambo MMed); Hôpital national du Mali, Bamako, Mali (A Sidibe MD); University of Newcastle at Yaoundé Central Hospital Yaoundé, Cameroon (E Sobngwi MD); School of Pharmacy, Jimma University, Jimma, Ethiopia (MTAngamo BPharm); Sheffield Teaching Hospitals and University of Sheffield, Royal Hallamshire Hospital, Sheffield, UK (S Tesfaye MD); Institute of Cardiovascular Science, Division of Medicine, University College London, London, UK

(J SYudkin FRCP); Clinique Médicale II, Centre de diabétologie Marc Sankale, Hôpital Abass Ndao, Dakar, Senegal (M N Mbaye MD); and Eastern Virginia Medical School,

Norfolk, VA, USA (E S Siraj MD)

Correspondence to: Prof Rifat Atun, Harvard TH Chan School of Public Health, Harvard

University, Boston, MA 02115,

ratun@hsph.harvard.edu See Online for appendix 1 For more on NCD-RisC see http://ncdrisc.org/

For more on H3Africa see http://hheafrica.org

\section{Population trends and ageing}

The population aged $20-79$ years in sub-Saharan Africa is projected to increase from 441 million in 2015 to 926 million in 2040. ${ }^{51}$ Given the association between diabetes and older age, this increase will be an important driver of the increase in the number of people with diabetes. Furthermore, increasing life expectancy is a notable feature of populations in sub-Saharan Africa, especially in countries with a high HIV prevalence and in which life expectancy is increasing with the rollout of antiretroviral therapies. ${ }^{52}$ Although large-scale, high quality studies from sub-Saharan Africa are scarce, a 2016 systematic review ${ }^{20}$ of studies published between 2000 and 2015 estimated the prevalence of diabetes to be $13.8 \%$ $(95 \%$ CI 13 2-14.3) in those aged 55 years or older. Given the potential impact of an ageing population on the prevalence of diabetes in sub-Saharan Africa, it is essential for health-system planning that high-quality, local information is obtained on the association between ageing and diabetes. See figure 1 for an analysis of the association between diabetes and age in sub-Saharan Africa.

\section{Type 1 diabetes in sub-Saharan Africa}

Type 1 diabetes can occur at any age, with a peak onset around the time of puberty in western countries. A later peak from around 15-25 years is often reported in Africa. ${ }^{53}$ With increasing age, the clinical distinction between types of diabetes can also seem blurred, which further compounds the issue of ascertaining the true lifetime prevalence of type 1 diabetes.

The incidence of type 1 diabetes began to rise in western populations around the middle of the last century, and is still increasing in many parts of the world..$^{54}$ The rapidity of this increase points to environmental causes superimposed upon genetic susceptibility. For example, Ethiopian immigrants to Israel developed type 1 diabetes at higher rates than their counterparts in Ethiopia; this is thought to be due to genetically predisposed individuals being exposed to new environmental triggers in Israel. ${ }^{55}$ Earlier age of onset is associated with higher degrees of genetic susceptibility (panel 3), and the secular trend towards earlier onset in western populations suggests increased penetrance of the disease; a similar shift has also been reported in Rwanda. ${ }^{56}$

The incidence of type 1 diabetes varies widely with geography, and a difference in the incidence of childhood type 1 diabetes of more than 350 times between populations has been described (from an age-adjusted incidence of $0 \cdot 1$ per 100000 population per year in China to 36.8 per 100000 population per year in Sardinia). ${ }^{57}$ Therefore, the incidence from one area cannot be easily extrapolated to another. Unfortunately, information about prevalence and incidence from sub-Saharan Africa is scarce. For example, estimates used in the 2015 IDF Atlas ${ }^{25}$ were extrapolated from studies in only five countries (Ethiopia, Nigeria, Rwanda, Tanzania, and Zambia). From the existing evidence, it seems that the incidence of type 1 diabetes in sub-Saharan Africa is relatively low compared with many other parts of the world. However, small poorquality studies, and the limited ability of health systems to diagnose the condition, means that the true prevalence remains obscure. ${ }^{58}$ Indeed, recent evidence from Rwanda suggests that the low apparent prevalence of type 1 diabetes in sub-Saharan Africa is very likely to represent failure of diagnosis or high mortality in diagnosed cases, because numbers increase sharply when interventions become available (see appendix 2 for profile of Rwanda). ${ }^{59,60}$ An additional limitation of studies is that estimates have generally focused on children younger than 15 years and therefore do not account for a substantial proportion of people with disease onset after this age. The only published prevalence figure to cover the entire young adult age group came from Rwanda and was $16 \cdot 4$ per 100000 population younger than 26 years. ${ }^{61,62}$ In Rwanda, there are $3 \cdot 5$ times as many cases of type 1 diabetes in the population younger than 26 years as in the population younger than 15 years.

\section{Other forms of diabetes in sub-Saharan Africa}

Other forms of diabetes in the region might constitute a greater proportion of cases than the $5 \%$ seen in HICs. ${ }^{26}$ Of particular note, in Ethiopia, many cases of diabetes that had previously been considered to be type 1 are now being reconsidered in light of antibody studies that have not shown the presence of antibodies typical of the disease. ${ }^{53}$

Malnutrition-related diabetes has been reported in subSaharan Africa, but its classification as a distinct subtype has been controversial, despite several cases being reported in the literature. ${ }^{50,53}$ Also known as fibrocalculous pancreatic diabetes, malnutrition-related diabetes is usually seen in underweight, malnourished patients and is characterised by severe hyperglycaemia without ketosis, high insulin requirements, and the absence of autoimmunity.

Variant forms of diabetes have been described in people of African descent since the 1950s. Reports from African American populations indicate that so-called variant diabetes is seen in new-onset patients, typically middleaged, overweight, and with a family history of type 2 diabetes in $80 \%$ of cases. Despite presentation with ketosis, such individuals can usually be managed without insulin. $^{63}$ The extent to which this represents a true variant of diabetes remains uncertain, and it is clear that there is the need for more detailed investigation of the pathophysiology of diabetes in people of African descent.

We discuss gestational diabetes in sub-Saharan Africa in panel 4 .

\section{Complications of diabetes \\ Overview}

As discussed extensively in panel 2, there is insufficient high-quality evidence to estimate the burden of diabetes in sub-Saharan Africa or to obtain reliable data about 
Panel 4: Gestational diabetes and hyperglycaemia in pregnancy

\section{Causes}

Relative insensitivity to insulin is a normal feature of pregnancy and gestational diabetes occurs when pregnancy results in overt hyperglycaemia. Because a fetus exposed to hyperglycaemia overproduces insulin, which can lead to macrosomia, obstetric difficulties might result. ${ }^{64}$ In addition to being caused by gestational diabetes, hyperglycaemia in pregnancy might also be due to pre-existing diabetes or diabetes that manifests for the first time during pregnancy. Maternal BMI and risk of gestational diabetes are highly correlated ${ }^{65}$ and hyperglycaemia in pregnancy is becoming increasingly common in parallel with the increased prevalence of diabetes and obesity in the background population. Increasing global trends in maternal overweight and obesity have been reviewed extensively by Poston and colleagues. ${ }^{65}$ In sub-Saharan Africa, Demographic and Health Surveys show wide variation in the prevalence of obesity in women of childbearing age, ranging from $0.7 \%$ in Madagascar to $26.8 \%$ in Lesotho. Although maternal underweight has previously been of great concern in the region, it is now less common than excess weight in women of childbearing age. ${ }^{66}$

\section{Prevalence}

The global prevalence of hyperglycaemia in pregnancy was estimated to be $16.9 \%$ in 2013 , equating to 21.4 million livebirths. ${ }^{65}$ More than $90.0 \%$ of cases were estimated to occur in low-income and middle-income countries, and $16.0 \%$ were attributed to pre-existing diabetes or diabetes manifesting for the first time during pregnancy, leaving around $64.0 \%$ due to gestational diabetes. However, the prevalence might vary by region depending on the diagnostic criteria used. ${ }^{67}$

Unfortunately, studies reporting the different causes of hyperglycaemia in pregnancy in sub-Saharan African countries are rare.

Scarce information exists about the prevalence of gestational diabetes in sub-Saharan Africa. A 2014 systematic review ${ }^{67}$ of gestational diabetes in the African continent found a prevalence of gestational diabetes ranging from $0 \%$ in Tanzania to $13.9 \%$ in Nigeria. The authors comment that "it is alarming that very little appears to be known about gestational diabetes in Africa", and only six countries (five in sub-Saharan Africa) provided data of sufficient quality for inclusion in the review. ${ }^{67}$ Additionally, diagnostic criteria differed between studies and increased prevalence was seen in studies done after the year 2000 and in those that used more current diagnostic criteria.

\section{Diagnosis of gestational diabetes}

It is recommended that screening for gestational diabetes is done between 24 weeks and 28 weeks of gestation with the $75 \mathrm{~g}$ oral glucose tolerance test (OGTT). However, differences exist between organisations' diagnostic criteria for gestational diabetes, and criteria for gestational diabetes have changed over time. ${ }^{67}$ This variation in criteria makes comparing studies and synthesising results from studies challenging, both within sub-Saharan Africa and globally. For example, a study ${ }^{68}$ of around 1000 pregnant women screened for gestational diabetes between 24 weeks and 34 weeks of gestation in a single clinic in Nigeria with the OGTT found the prevalence of gestational diabetes to be $3.8 \%$ when 1999 WHO criteria $^{69}$ were used, $8 \cdot 1 \%$ when 2013 WHO criteria $^{70}$ were used, and $8.6 \%$ when International Association of Diabetes and Pregnancy Study Groups Consensus Panel (IADPSG) criteria ${ }^{71}$ were used.

After the results of the HAPO study ${ }^{72}$ were reported in 2008, many groups revised their guidelines. In particular, the IADSPG and WHO 2013 (similar to IADSPG) criteria differ from older criteria or those used by other organisations; only one abnormal value, rather than two, is needed to diagnose gestational diabetes, and the fasting glycaemic thresholds used to diagnose glycaemia are lower than previous criteria. ${ }^{67}$ The new criteria will probably result in an increased prevalence of gestational diabetes, with knock-on effects on burden to health systems. For a full review of the diagnostic criteria for gestational diabetes, see the review by Ma and colleagues. ${ }^{64}$ It should also be noted that the HAPO study - on which the IADSPG and WHO 2013 criteria are based-investigated outcomes of hyperglycaemia in pregnancy in a non-African population, and outcomes might be different in populations that are distinct from the original study population. ${ }^{73}$ Further research is needed to ascertain whether the new IADSPG and WHO 2013 criteria are applicable to populations in sub-Saharan Africa in terms of their ability to predict adverse maternal and offspring outcomes.

Risk factors for gestational diabetes in sub-Saharan Africa, as elsewhere, are gestational diabetes in a previous pregnancy, family history of type 2 diabetes, previous stillbirth or child with macrosomia, and maternal age of 30 years or older. It is not clear whether sub-Saharan African women are at increased risk of gestational diabetes compared with other ethnic groups. In a multi-ethnic society such as the USA, women from southeast Asia were found to develop gestational diabetes at lower BMI than women of European, Hispanic American, or African American origin, and it was estimated that two-thirds of gestational diabetes cases in African Americans could be prevented if all women were of normal weight when they entered pregnancy. ${ }^{74}$

\section{Complications and treatment of hyperglycaemia in pregnancy} Complications include pregnancy-induced hypertension and pre-eclampsia, antepartum haemorrhage, complications of labour, preterm birth, birth trauma, congenital anomalies, and high perinatal mortality. ${ }^{64,65}$ Women with gestational diabetes are at a high risk of developing subsequent type 2 diabetes, and their offspring have increased susceptibility to glucose intolerance and obesity later in life. Additionally, the risk of diabetic retinopathy during pregnancy is nearly twice that in the non-pregnant state. ${ }^{75}$ The rate of complications is greater in pregnancies with preexisting diabetes than in those without. ${ }^{64,65.76}$

(Continues on next page)
For more on the RODAM study

see http://www.rod-am/eu/ content/objectives See Online for appendix 2 
(Panel 4 continued from previous page)

Relatively little is known about the maternal or offspring outcomes of pregnancy in women with pre-existing diabetes in sub-Saharan Africa, other than from a few previous studies, ${ }^{77-79}$ which had small sample sizes or were published years ago and so have little relevance to the current situation in the region. The increasing prevalence of obesity, and its associations with gestational or type 2 diabetes, in women who are of reproductive age, could have a great impact on both women and their offspring. That so little is known about these issues in sub-Saharan Africa is cause for serious concern. Treating hyperglycaemia during pregnancy in women in sub-Saharan Africa might be no different to treating women in other countries. However, all studies identified by this Commission about the management of hyperglycaemia in pregnancy in sub-Saharan African populations have been observational, ${ }^{78,80-82}$ randomised trials comparing treatments in sub-Saharan Africa are urgently needed. trends over time. The same is true when considering the burden of diabetes-related complications. In particular, detection bias probably has a large effect on the reporting of complications of diabetes in countries where health systems are not sufficiently developed to provide highquality services, and where health-care-seeking behaviour is low. Additionally, evidence suggests that hyperglycaemia in patients in sub-Saharan Africa is detected at a later stage than in patients in HICs. ${ }^{10}$ Therefore, given the longer lead time of hyperglycaemia before treatment is given, patients in sub-Saharan Africa might appear more susceptible to complications than their peers in other countries. A scarcity of data systems in many countries is a major hurdle to adequate documentation of complication rates; WHO found that only $17 \%$ of countries in sub-Saharan Africa had any form of diabetes registry (although WHO did not report the geographical coverage of, or the quality of, information in those registries). ${ }^{83}$ Additionally, the quality of the data reported will inevitably vary in line with the ability of health services to record and retrieve information related to complications of diabetes. Although we present in the sections below the highest-quality evidence available for illustrative purposes, the evidence is probably not a good representation of complications in the entire region. We do not discuss interactions between diabetes and infectious diseases, which are of particular relevance to sub-Saharan Africa, where high rates of infectious disease coexist with an increasing prevalence of NCDs including diabetes. These interactions have been extensively reviewed elsewhere; ${ }^{8487}$ comprehensive coverage of these areas are beyond the remit of this Commission.

\section{Death}

The 2015 GBD study $^{9}$ estimated that, in 2013, diabetes contributed to an average of 145189 deaths (uncertainty interval 129914-164809) in sub-Saharan Africa, which amounted to $1.8 \%(1.68-1 \cdot 97)$ of all deaths in the region. These figures should be interpreted with caution given the inadequate recording of cause of death in subSaharan Africa. Indeed, given the prevalence of diabetes in the region, and the inadequacy of many health systems to effectively diagnose and treat diabetes and its complications, this estimate seems very low.
Little is known about the causes of death due to chronic complications of diabetes in sub-Saharan Africa. ${ }^{10}$ This knowledge deficit is due to the scarcity of high-quality vital registration systems in sub-Saharan Africa and poor diagnostic facilities. Some countries in the region use verbal autopsies in sample populations to determine the cause of death, which give estimates at the population level. A 2014 study $^{88}$ from the INDEPTH network of Health and Demographic Surveillance System sites suggested that diabetes contributed little to mortality caused by NCDs in Africa compared with that caused by cardiovascular diseases and cancers. However, this method might not have captured diabetes as a contributor to death if it was not ascertained as a direct cause of death.

There is also insufficient data from the region on acute mortality due to diabetes, although the Commissioners' experiences and opinions suggest that acute mortality caused by diabetes in sub-Saharan Africa is most often due to undiagnosed or inadequately treated type 1 diabetes, which rapidly progresses to diabetic ketoacidosis and death. Early reports from sub-Saharan Africa document a very high mortality for type 1 diabetes. In Mali during the $1990 \mathrm{~s}, 50 \%$ of patients died within 2 years of a type 1 diabetes diagnosis, ${ }^{89}$ and a study ${ }^{90}$ published in 2005 estimated the life expectancy of children younger than 15 years in Mozambique to be 3.5 years after diagnosis; a child in a rural area who developed diabetes was unlikely to survive for more than 1 year. Data on mortality of people with type 1 diabetes from some other countries suggested less severe outcomes. In Soweto, South Africa, mortality due to type 1 diabetes was $16 \%$ in the period of 10 years between 1982 and 1992 (with half of the deaths being from renal failure and the remaining deaths being from ketoacidosis, hypoglycaemia, or sepsis), although a follow-up study showed $43 \%$ mortality after 20 years of follow-up. ${ }^{81}$ Data from Rwanda in 2015 suggested a 5 year survival of $93 \cdot 8 \% .{ }^{25}$ However, it is unlikely that these data reflect the true mortality of type 1 diabetes in Rwanda because a substantial amount of data were missing and participants could potentially have been subject to the Hawthorne effect. It is possible that mortality for type 1 diabetes in Rwanda could have been as high as three times the crude mortality rate of 13.9 per 1000 patient-years..$^{25}$ 
Although reliable data from sub-Saharan Africa are not available, the small number of studies that have been done can still be taken to reflect a state of care that is far below standards seen in more affluent parts of the world, where life expectancy for young people diagnosed with type 1 diabetes is now only a few years less than that of the general population. ${ }^{91}$

\section{Chronic complications of diabetes}

The chronic complications of diabetes affect blood vessels and are conventionally subdivided into macrovascular and microvascular; however, they also affect other tissues, including nerves and the optic lens. Little is known about the prevalence, age of onset, or rate of progression of diabetic complications in subSaharan Africa, and most evidence comes from small, single-country, single-centre, and somewhat out-of-date studies. A systematic review ${ }^{92}$ that used GBD methods calculated that the non-fatal burden of diabetes in total years lost due to disability in South Africa in 2009 was 73714; 42919 (58.2\%) from diabetes alone, $13458(18 \cdot 3 \%)$ from retinopathy, 4527 (6.1\%) from amputations, $7233(9.8 \%)$ from attributable stroke disability, and 5577 (7.6\%) from attributable ischaemic heart disease disability. Additionally, the GBD 2015 study ${ }^{9}$ estimated that diabetes contributed to 5556560 (uncertainty interval 4753194-6442898) DALYs in 2013, which corresponded to $1.05 \%(0 \cdot 94-1 \cdot 16 \%)$ of all DALYs in sub-Saharan Africa. Such estimates are necessarily imprecise and, as previously discussed, seem low given the estimated prevalence of diabetes in sub-Saharan Africa and the poor access to health systems for management of hyperglycaemia.

The scarcity of reliable data is highlighted as a limitation in all reports of specific complications in subSaharan Africa. For example, a 2011 systematic review ${ }^{26}$ of the prevalence of chronic complications of diabetes in the region found only 23 eligible studies. The recorded prevalence of retinopathy varied from $7 \%$ in Kenya to $63 \%$ in South Africa, of neuropathy from $27 \%$ in Cameroon to $66 \%$ in Sudan, and of microalbuminuria from $10 \%$ in Tanzania to $83 \%$ in Nigeria. Macrovascular complications were not covered, and a search of the literature done by the Commission did not return any studies addressing the prevalence of cardiovascular complications. The most recent regional narrative review was done by Levitt ${ }^{10}$ in 2008, who noted that ischaemic heart disease was less common in indigenous Africans with diabetes (5-8\% based on electrocardiogram stress tests and $4 \%$ based on history) than in their counterparts of European heritage (23\% based on positive history). Levitt also noted that there was little available evidence on the risk of stroke in people with diabetes.

The available information indicates that vascular complications develop sooner after a diabetes diagnosis in sub-Saharan African patients than in those from other parts of the world, ${ }^{10}$ which is an inevitable consequence of late diagnosis and poor glucose control. Thus, if reliable evidence was available, we suspect that the prevalence and severity of complications of diabetes in the region would far surpass reports from studies of HICs. This deduction is of concern, given that health systems in sub-Saharan Africa are already struggling.

\section{Diabetic eye disease}

The manifestations of diabetes that affect sight are diabetic retinopathy, proliferative retinopathy, and maculopathy, all of which are preventable and treatable before vision is lost. ${ }^{93}$ Cataracts are prevalent among patients with diabetes in Africa, but much of the literature has focused on diabetic retinopathy, which is where we focus our discussion.

Globally, diabetic retinopathy accounts for $2.6 \%$ (95\% CI 2-2-3.4) of all blindness, ${ }^{94}$ and age-standardised prevalence of retinopathy as a cause of blindness was found to be highest in sub-Saharan Africa, at $0.14 \%$ $(0 \cdot 10-0 \cdot 20) .^{94}$ The most up-to-date systematic review ${ }^{95}$ of the prevalence of diabetic retinopathy in people with diabetes in sub-Saharan Africa was published in 2013. The authors of that review ${ }^{95}$ included 62 studies from 21 countries in the region and, accepting recognised differences in diagnoses and sampling techniques, the prevalence in population-based surveys was $30 \cdot 2-31 \cdot 6 \%$ for diabetic retinopathy, $0.9-1.3 \%$ for proliferative diabetic retinopathy, and $1 \cdot 2-4 \cdot 5 \%$ for any maculopathy. Those numbers were roughly similar to the global reported prevalence, ${ }^{96}$ which is surprising given the poor access to services for the diagnosis and treatment of hyperglycaemia in sub-Saharan Africa, although it is likely that poor access to health systems and reporting in subSaharan African populations result in underestimation of prevalence.

\section{Diabetic nephropathy}

Globally, the contribution of chronic kidney disease to deaths nearly doubled between 1990 and 2010, although improved ascertainment and ageing populations might have contributed to this increase. ${ }^{97}$ In $2010,70 \%$ of patients with end-stage renal failure were predicted to live in low-income countries (LICs). Hypertension was the leading cause of death from kidney disease worldwide in 2010, ${ }^{77}$ but the number of cases attributable to hypertension was decreasing by 2013 , whereas cases attributable to diabetes were increasing. ${ }^{98}$

The true prevalence of both chronic kidney disease and diabetic nephropathy in sub-Saharan Africa remains uncertain because of the lack of population-based surveys and diabetes registries. However, evidence from a systematic review and meta-analysis ${ }^{99}$ of studies involving 64307 people estimated the prevalence of chronic kidney disease in sub-Saharan Africa to be $13.9 \%$ (95\% CI $12 \cdot 2-15 \cdot 7)$. The mean age of people in that study ${ }^{99}$ was 41.4 years (SD 9.9); 46494 (72\%) people had diabetes, 2765 (4\%) were obese, 37169 (58\%) were HIV-positive, 
and 7845 (12\%) had hypertension. These findings should be interpreted with caution, since only three of 90 studies were considered high quality. Notably, the estimated prevalence of diabetes was decreased to $6 \%$ in the 21 studies deemed of medium or good quality. ${ }^{99,100}$ Similar caution is needed with regards to evidence about the prevalence of nephropathy in patients with diabetes, which has in most studies been based on the presence of proteinuria. A systematic review ${ }^{101}$ identified 32 studies from 16 African countries, only two of which were population-based. That study suggested a prevalence of proteinuria in patients with diabetes as high as 95\% at 10 years of follow-up, with an $18.4 \%$ mortality from nephropathy at 20 years of follow-up.

Studies $^{102}$ have shown that African Americans have twice the risk of end-stage renal disease compared with people of European descent, even after correction for socioeconomic and clinical risk factors. This increased risk appears to be due in part to inheritance of an apolipoprotein L1 (APOL1) gene variant, of which the high prevalence in west Africa might have been driven by selection for the protection it confers against some variants of trypanosomiasis. ${ }^{102}$ APOL 1 has been associated with accelerated progression of several types of renal disorder, including increased susceptibility to HIVinduced nephropathy, and might have a similar role in diabetic nephropathy. ${ }^{103}$ When factors such as hypertension, HIV, genetic predisposition, and diabetes are combined, as is often the case, renal damage is likely to be accelerated. ${ }^{104}$ In fact, the average age of onset of end-stage renal disease in sub-Saharan Africa is estimated to be 20 years younger than that in HICs. ${ }^{104}$

\section{Diabetic neuropathy and diabetic foot}

Diabetic peripheral neuropathy is probably the most common complication of diabetes globally, affecting more than $50 \%$ of patients with diabetes. ${ }^{105,106}$ However, estimates vary depending on the diagnostic and epidemiological methods used. Diabetic peripheral neuropathy can be painless or, less commonly, painful. ${ }^{107}$ Although well designed, large, up-to-date, population-based studies are not available, and this summary should be interpreted with that in mind, published data from countries in subSaharan Africa suggest that diabetic peripheral neuropathy is common in the region. ${ }^{105}$ In the USA, the prevalence of diabetic foot ulcers was estimated to be $8 \%$ of patients with diabetes, ${ }^{108}$ whereas the prevalence in sub-Saharan Africa is thought to be higher. For example, the prevalence of diabetic foot ulcers was estimated to be $15 \%$ in Tanzania, ${ }^{109} 13 \%$ in Cameroon, ${ }^{110}$ and $9.5 \%$ in Nigeria. ${ }^{111}$ In hospitalised patients with diabetes, as would be expected, the prevalence of diabetic foot ulcers is even higher, ${ }^{110,111}$ although limitations of study size apply. The clinical features of painful diabetic peripheral neuropathy are similar to those in patients outside of sub-Saharan Africa and include poor quality of life, insomnia, and depression. ${ }^{112}$
Although, once again, evidence is sparse and not recent enough to draw clear conclusions, peripheral neuropathy is thought to be the principal underlying risk factor for foot ulceration in patients with diabetes in sub-Saharan Africa. ${ }^{113,114}$ This differs from HICs, in which peripheral arterial disease is closely associated with the development of diabetic foot disease. ${ }^{115}$ The pattern seems to be changing, however, with the prevalence of peripheral arterial disease increasing rapidly in people with diabetes in sub-Saharan Africa. Compared with a prevalence of less than $10 \%$ in the 1990s, more recent studies have shown an increased prevalence of peripheral arterial disease of between $20 \%$ and $54 \% .^{116-118}$

In addition to diabetic peripheral neuropathy and peripheral arterial disease, multiple environmental factors are associated with both the occurrence and severity of diabetic foot disease-for example, bare-foot walking (which might be cultural or related to an inability to afford shoes); ill-fitting shoes; or rodent bites on feet, particularly in people who sleep on the floor. ${ }^{113,119}$

In sub-Saharan Africa, amputations are frequent outcomes in patients with diabetic foot ulcers. Around a third of such amputations have been associated with neuro-ischaemic lesions, progressive infection, or both. ${ }^{120}$ The in-hospital mortality of patients with severe foot ulcers (Wagner score $>4$ ) managed without surgery or amputation can be as high as $54 \% .{ }^{121}$ Amputation rates might also be lower than expected because of difficulty in obtaining consent for surgery. Sadly, some patients with severe diabetic foot ulcers discharge themselves from hospital against medical advice, putting themselves at high risk of severe sepsis and death at home.

\section{Arterial disease}

Cardiovascular disease is modulated by three major preventable risk factors other than smoking (the prevalence of which is increasing rapidly in Africa ${ }^{122,123}$ ): hypertension, diabetes, and hyperlipidaemia. Although obesity largely manifests risk through its effects on these factors, it is still classed by many investigators as a modifiable risk factor of cardiovascular disease and is thus included in this discussion. Together, these risk factors are thought to account for most deaths from cardiovascular causes. According to GBD, ${ }^{97}$ hypertension remains the leading risk factor worldwide, but diabetes (and obesity) replaced cholesterol in second place between 1980 and 2010 for all categories other than ischaemic heart disease, with the mortality burden shifting from HICs to LMICs. In sub-Saharan Africa, obesity and hypertension are thought to be the most common cardiovascular risk factors, although issues of insufficient reliable data apply. ${ }^{124}$ We have discussed obesity earlier in this report. The prevalence of high blood pressure has increased rapidly in the past 2-3 decades and, according to the WHO STEPwise approach to surveillance (STEPS) surveys done in 
sub-Saharan Africa, ${ }^{125}$ the prevalence of high blood pressure ranges from $19.3 \%$ in Eritrea to $39.6 \%$ in Seychelles. It is estimated that 150 million people living in sub-Saharan Africa will be treated for hypertension by 2025 compared with 80 million in 2010, an increase attributed to excessive alcohol consumption, reduced physical activity, and adoption of western diets and other features of the economic transition. ${ }^{125}$

The extent of co-association of cardiovascular risk factors in individuals with diabetes is even less well known. Recent studies have estimated that, among patients with diabetes in sub-Saharan Africa, the prevalence of hypertension ranges from $44 \%$ to $76 \% .{ }^{126-129}$ Little is known about the prevalence of hyperlipidaemia in patients with diabetes in sub-Saharan Africa, and the prevalence of metabolic syndrome among people with diabetes in clinical settings in the region ranged from $25 \%$ to more than $90 \%$, depending on the criteria used. ${ }^{130}$ Insufficient high-quality information is also a major limitation when reviewing the features of people presenting with cardiovascular disease in sub-Saharan Africa. For example, a systematic review ${ }^{131}$ of myocardial infarction in the region found only seven studies from five countries that satisfied all inclusion criteria. Nevertheless, information from another review ${ }^{132}$ illustrated the heterogeneity of risk factors in patients presenting with coronary disease: $41 \cdot 0-66 \cdot 3 \%$ for hypertension, $22 \cdot 5-40 \cdot 0 \%$ for diabetes, $8 \cdot 8-67 \cdot 3 \%$ for hyperlipidaemia, $11 \cdot 8-44.0 \%$ for smoking, and $27 \cdot 0-80 \cdot 0 \%$ for obesity. Sample size was exceedingly small in all the studies included in the review, ${ }^{132}$ ranging from 30 patients to 169 patients, probably reflecting the inadequacy of record keeping and storage.

A worrying feature of the changing pattern of cardiovascular disease in sub-Saharan Africa is the proportion of deaths in people younger than 70 years, which is still increasing in the region despite decreasing in many HICs; deaths attributed to cardiovascular disease in people younger than 70 years contributed to $21 \%$ of total deaths in HICs compared with $58 \%$ of total deaths in subSaharan Africa in 2013.' Thus, cardiovascular disease seems to be affecting younger, more economically productive people in sub-Saharan Africa. The scale of the cardiovascular disease epidemic likely to affect subSaharan Africa awaits clearer definition, and so too does the pattern. However, cerebrovascular disease is likely to contribute substantially to the burden of cardiovascular disease given that it is thought to be responsible for around $11 \cdot 23 \%$ (95\% CI 10.92-11.57) of all deaths worldwide. ${ }^{2}$ Stroke is potentially preventable, and a systematic review ${ }^{133}$ of worldwide stroke incidence showed a $42 \%$ reduction in incidence in HICs in the four decades from 1970 to 2008. By contrast, the incidence of stroke has increased by $100 \%$ in LMICs. ${ }^{133}$ Little is known about the prevalence of stroke in patients with diabetes in subSaharan Africa, although early reports suggest that it is low. For example, in a large study ${ }^{134}$ covering the period from 1999 to 2012 in a major hospital in urban Cameroon, and involving 1688 patients admitted for stroke, the prevalence of diabetes was $12 \cdot 8 \%$. However, the prevalence is likely to vary greatly depending on geographical location.

\section{Clinical challenges of diabetes in sub-Saharan \\ Africa \\ Diagnosing diabetes in sub-Saharan Africa}

The clinical challenges of diabetes in sub-Saharan Africa are numerous, yet, despite differing levels of development and population structure, countries within the region face similar challenges concerning screening, diagnosis, and management. In this section we consider issues of clinical relevance, wherein common themes in the inability to provide quality care are scarcity of knowledge, inability to reliably access drugs, and poor access to treatments for complications. Health system considerations will be addressed in more detail in subsequent sections.

Poor awareness of diabetes, both at population and health-care-profession levels, means diagnosis is often delayed. For people with type 2 diabetes, this delay will generally result in an unequivocally elevated random glucose at presentation. ${ }^{10,39,135}$ Choosing the correct cutoff point or test to use for diagnosis of diabetes (panel 2) is therefore of less immediate relevance in the context of routine clinical diagnosis and care in this setting, but the question of whether the American Diabetes Association (ADA) guidelines ${ }^{136,137}$ for diagnosis of diabetes can be applied to a sub-Saharan African population will become increasingly important in the future as earlier detection of diabetes becomes feasible. It is worth noting that, although $\mathrm{HbA}_{1 \mathrm{c}}$ is increasingly used as a diagnostic tool in HICs, its use is likely to face challenges in Africa given that it relies on integrity of red blood cells, which can be affected by several conditions that are prevalent in subSaharan Africa, such as haemolysis from malaria or sickle cell disease.

Diagnosis of diabetes is complicated by the poor availability of diagnostic equipment in clinics, laboratory facilities to process samples, and transport between clinics and laboratory facilities (table 3). Therefore, rather than rely on the gold-standard measures put forward by the ADA, many practitioners rely on capillary blood glucose measurements made with point-of-care instruments and urine strips. However, even these simple tests are often unavailable. For example, Beran and colleagues ${ }^{90,139}$ found that urine glucose strips were available in only $18 \%$ and blood glucose meters in only $21 \%$ of health-care facilities in Mozambique. Blood glucose meters were available in 13\% (urine glucose strips in 54\%) of facilities in Mali and in 49\% (urine glucose strips in 61\%) of facilities in Zambia. ${ }^{90,139}$

For people with type 1 diabetes, a delay in diagnosis can prove fatal. Unfortunately, given that in LICs the symptoms of type 1 diabetes-rapid weight loss, fatigue, abdominal pain, and confusion-can easily be mistaken for AIDS or cerebral malaria, ${ }^{140,141}$ and that the condition is 


\begin{tabular}{|c|c|c|c|c|c|c|c|c|c|c|c|}
\hline & $\begin{array}{l}\text { Cost per } \\
\text { person with } \\
\text { diabetes(U\$)* }\end{array}$ & $\begin{array}{l}\text { Facilities offering } \\
\text { diabetes } \\
\text { management* }^{*}\end{array}$ & $\begin{array}{l}\text { Readiness to } \\
\text { provide diabetes } \\
\text { servicest }\end{array}$ & $\begin{array}{l}\text { Guidelines } \\
\text { available to } \\
\text { treat diabetes* }\end{array}$ & $\begin{array}{l}\text { At least one } \\
\text { trained member } \\
\text { of staff* }\end{array}$ & $\begin{array}{l}\text { Blood } \\
\text { glucose } \\
\text { testing* }\end{array}$ & $\begin{array}{l}\text { Urine } \\
\text { dipstick } \\
\text { proteins* }\end{array}$ & $\begin{array}{l}\text { Urine } \\
\text { dipstick } \\
\text { ketones* }^{*}\end{array}$ & $\begin{array}{l}\text { Metformin } \\
\text { capsules* }\end{array}$ & $\begin{array}{l}\text { Glibenclamide } \\
\text { capsules* }^{*}\end{array}$ & $\begin{array}{l}\text { Injectable } \\
\text { insulin* }\end{array}$ \\
\hline Benin (2013) & $73 \cdot 0$ & $33 \%$ & $44 \%$ & $10 \%$ & $5 \%$ & $31 \%$ & $52 \%$ & $26 \%$ & $22 \%$ & $29 \%$ & $7 \%$ \\
\hline $\begin{array}{l}\text { Burkina Faso } \\
\text { (2012) }\end{array}$ & $91 \cdot 0$ & $42 \%$ & $43 \%$ & $27 \%$ & $19 \%$ & $14 \%$ & $70 \%$ & $25 \%$ & $6 \%$ & $6 \%$ & $4 \%$ \\
\hline Kenya (2013) & $82 \cdot 4$ & $37 \%$ & .. & .. & .. & .. & .. & .. & .. & .. & .. \\
\hline $\begin{array}{l}\text { Mauritania } \\
\text { (2013) }\end{array}$ & $89 \cdot 6$ & $17 \%$ & .. & $38 \%$ & $16 \%$ & $25 \%$ & $33 \%$ & $26 \%$ & $9 \%$ & $8 \%$ & $12 \%$ \\
\hline $\begin{array}{l}\text { Sierra Leone } \\
(2012)\end{array}$ & $184 \cdot 4$ & $23 \%$ & $51 \%$ & $25 \%$ & $6 \%$ & $43 \%$ & $82 \%$ & $64 \%$ & $46 \%$ & $35 \%$ & $20 \%$ \\
\hline $\begin{array}{l}\text { Tanzania } \\
(2012)\end{array}$ & $95 \cdot 5$ & $12 \%$ & $51 \%$ & $30 \%$ & $34 \%$ & $40 \%$ & .. & .. & $57 \%$ & $52 \%$ & $34 \%$ \\
\hline $\begin{array}{l}\text { Uganda } \\
\text { (2013) }\end{array}$ & No estimate & $34 \%$ & $60 \%$ & $83 \%$ & $31 \%$ & $80 \%$ & $61 \%$ & .. & $49 \%$ & $55 \%$ & $15 \%$ \\
\hline Zambia (2010) & 186.6 & $32 \%$ & $57 \%$ & $43 \%$ & $20 \%$ & $51 \%$ & $67 \%$ & .. & $36 \%$ & $70 \%$ & $39 \%$ \\
\hline
\end{tabular}

Data are \% of facilities unless otherwise specified. Income status for each country (all are low income) was determined by data from the World Bank (2014). ${ }^{138 *}$ Data are from country Service Availability Readiness Assessment reports. †Data are from the International Diabetes Federation 2015 Diabetes Atlas. ${ }^{25}$

Table 3: Service delivery indicators for facilities offering diabetes diagnosis, management, or both, by low-income country

relatively rare, anecdotal reports and small studies suggest that diagnosis is often delayed. Indeed, Makani and colleagues $^{141}$ found that 21 (11\%) of 199 patients diagnosed with cerebral malaria in Tanzania actually had diabetes.

We discuss diagnosis of gestational diabetes in panel 4.

\section{Managing hyperglycaemia in sub-Saharan Africa}

Effective glycaemic control is essential for short-term wellbeing and long-term protection against complications. Available evidence suggests that many people with diabetes in sub-Saharan Africa fail to achieve adequate glycaemic control, although studies specifically addressing this issue have been small. ${ }^{142-144}$ Good glycaemic control is most likely to be achieved when a patient has reliable access to clinical services, when the availability of equipment to monitor control is good, when patients and health-care professionals have good knowledge about diabetes management, and when efficacious and affordable treatment is available and backed by adequate and effectively deployed measures of glucose control. In sub-Saharan Africa, barriers are experienced at each of these steps. Manne-Goehler and colleagues $^{11}$ found that only around one-fifth of overweight or obese people at high risk of diabetes remembered ever being offered blood glucose testing, that just over one-third of those who were identified as having diabetes remembered ever having a test done, and that only one-quarter were on medication.

The ability to monitor glycaemic control per se in subSaharan Africa is difficult, even in hospital settings. For example, in a survey of six countries done in $2011,{ }^{128}$ only around $47 \%$ (range $27 \cdot 5-81 \cdot 1$ ) of patients with diabetes had had $\mathrm{HbA}_{1 \mathrm{C}}$ measured in the 12 months before the study. In primary care settings, the situation is probably worse; however, we could not find any published data from sub-Saharan Africa. The availability of other methods for measuring glycaemia in the clinical setting was discussed earlier. As discussed in panel 2, the choice of test to monitor long-term glycaemic control is a key consideration and, although $\mathrm{HbA}_{1 \mathrm{C}}$ is the test of choice in many centres in sub-Saharan Africa, ${ }^{39}$ whether measurement of $\mathrm{HbA}_{1 \mathrm{c}}$ is the best test for a sub-Saharan African population is unclear. Other methods of monitoring glycaemic control, such as measuring concentrations of fructosamine or glycated albumin, ${ }^{17}$ might be beneficial in populations for whom $\mathrm{HbA}_{1 \mathrm{c}}$ is not reliable. These tests are not, however, widely available in sub-Saharan Africa, and their potential value in the region needs to be explored further.

Limited access to home blood glucose monitoring equipment adversely affects patient education and empowerment, which is a mainstay of diabetes management. ${ }^{145}$ The median cost of a blood glucose strip in seven African nations was US $\$ 0 \cdot 50$ (range $\$ 0 \cdot 20-\$ 1 \cdot 20$ ), and the yearly cost of consumables for minimal reasonable care to families with a child or young person with diabetes in these countries ranged from $74 \%$ to $377 \%$ (median 126\%) of per-capita gross national income. ${ }^{146}$

The first step of type 2 diabetes treatment in HICs is lifestyle advice, but few patients in sub-Saharan Africa receive such advice. Additionally, most people with type 2 diabetes require drug treatment, with the most commonly available drugs being metformin and sulfonylureas, or insulin for those who do not respond to treatment with these drugs. Affordability should not, in principle, be an obstacle to treatment, since these drugs are potentially available at low cost. Unfortunately, as discussed later in this report, supply-chain problems and price mark-ups are such that simple therapies are often unavailable or unaffordable at the patient level.

Newer and more expensive treatments are actively marketed in all parts of the world ${ }^{147}$ and might have advantages in certain subgroups of patients, ${ }^{148}$ although there are few outcome studies to show that they can 
achieve cost-effective reductions in morbidity or mortality compared with the three basic medications (metformin, sulfonylureas, and insulin). The pressing need, therefore, is to ensure that these basic medicines are available throughout sub-Saharan Africa and that clinicians are well educated about the ability to treat diabetes with simple agents (panel 1).

For type 1 diabetes, the essential therapy is insulin. Unfortunately, insulin and other components of care are often either unavailable in health-care systems or unaffordable to patients. These issues have been extensively reviewed elsewhere $e^{60,19}$ and are summarised in figure 2. Safe insulin storage is also a problem for many families who do not have access to refrigeration and so use clay pots for evaporative cooling. Encouragingly, evidence indicates that this method is effective in reducing storage temperatures towards standard room temperature of $20-25^{\circ} \mathrm{C} .{ }^{150}$ In addition to treating the disease, health-care providers need to recognise that the emotional impact on young people with type 1 diabetes and their families is often severe, especially in countries with relatively low health literacy. ${ }^{151}$ Knowledge can help to mitigate this burden, and, given the complexity involved in the management of type 1 diabetes, diabetes education of young patients, their families, and health professionals-tailored to culture, language, and education or knowledge levels-is critical to achieving good outcomes. Several non-governmental initiatives exist to provide care for people with type 1 diabetes in sub-Saharan Africa (appendix 1).

We discuss management of gestational diabetes in panel 4.

\section{Management of coexisting risk factors}

Overall, cardiovascular disease risk is logically addressed by treating overall risk rather than by focusing on hyperglycaemia alone. ${ }^{152}$ This approach presents challenges, but the Steno-2 study ${ }^{153}$ was a striking demonstration of the benefits of multifactorial intervention in type 2 diabetes. Wherever possible, people with diabetes should have regular assessments for all risk factors of cardiovascular disease. However, studies in sub-Saharan Africa have shown the difficulties of such concomitant services. For example, in a slum in Nairobi, Kenya, Werner and colleagues ${ }^{154}$ found that only $3.4 \%$ of people attended cardiovascular risk clinics on a regular basis during 34 months of follow-up. Adherence to recommended practice among medical professionals is also low, with many doctors in a cardiovascular risk clinic failing to follow guidelines for concomitant assessment of cardiovascular risk. ${ }^{155}$ Additionally, the guidelines for risk evaluation and reduction in sub-Saharan Africa are inconsistent, often focusing on single risk factors, and are generally adaptations of guidelines from other settings. ${ }^{156}$ More evidence from local settings is urgently needed to feed into local guidelines.
Although this Commission focuses on diabetes and total cardiovascular disease risk, we would be remiss if we did not mention that the reduction of cardiovascular disease in sub-Saharan Africa requires a concerted effort to reduce hypertension, given the high prevalence of hypertension in the region. A 2014 systematic review ${ }^{157}$ of 33 surveys involving more than 110000 participants in sub-Saharan Africa found a pooled prevalence of hypertension of $30 \%$, but only $27 \%$ of people with hypertension were aware of their status before the surveys, only $18 \%$ were on treatment, and only $7 \%$ had acceptably controlled blood pressure. In fact, the World Heart Federation ${ }^{158}$ puts both tobacco and blood pressure control in third place in its nine steps to reduce global burden of cardiovascular disease and does not mention control of hyperglycaemia. However, given that diabetes and hypertension often co-occur and interact synergistically to increase cardiovascular risk, the burden of diabetes is likely to increase in sub-Saharan Africa, and the adverse effects of diabetes go beyond those of cardiovascular disease, diabetes must be considered an important risk factor.

\section{Monitoring and managing complications of diabetes}

In view of the huge costs involved in managing the complications of diabetes, and the increasing burden of diabetes, strategies to prevent complications are desperately needed in sub-Saharan Africa. Adequate management of hyperglycaemia and other risk factors, and regular assessment for early evidence of complications, are the cornerstones of successful strategies to prevent microvascular and macrovascular complications. Diabetes registries have been highly successful platforms to drive improved outcomes in these regards in HICs, but issues with availability of technology and other necessary infrastructure mean that such registries are largely absent in sub-Saharan Africa. ${ }^{83}$

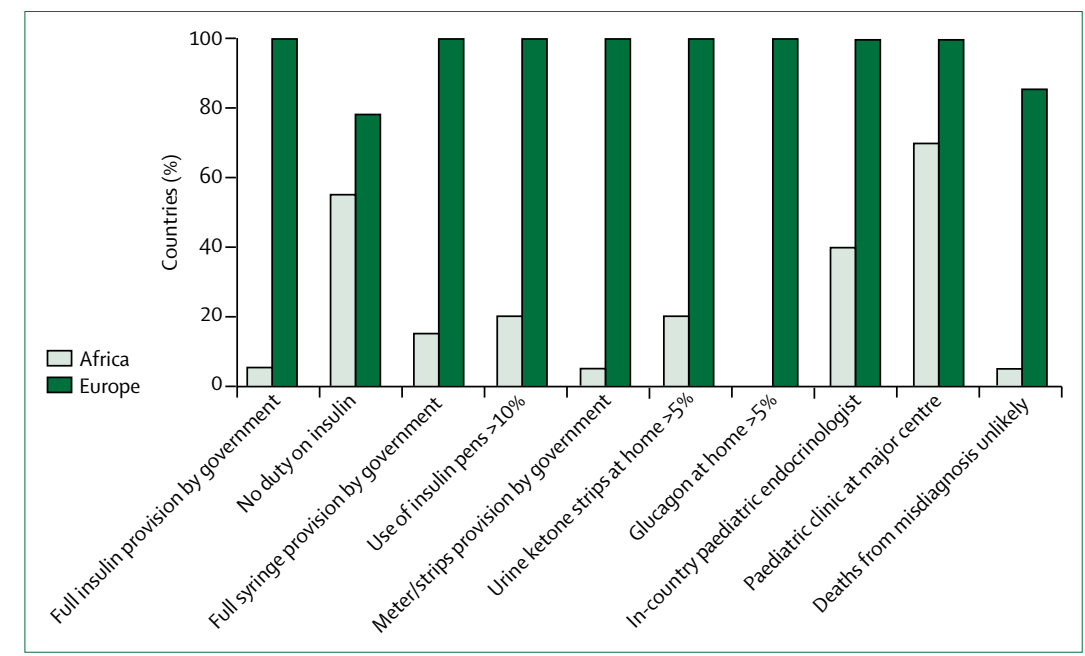

Figure 2: Provision of care for children with type 1 diabetes in 20 African and 14 European countries Percentages of study countries providing 12 key components of diabetes care are shown. Data are from a subanalysis of data from the 2015 study by Ogle and colleagues. ${ }^{60}$ 
The availability of management strategies to prevent progression or treat complications of diabetes is generally poor in sub-Saharan Africa. Challenges in prevention and management of diabetic retinopathy and nephropathy illustrate the issues in the region. Prevention and management of diabetic retinopathy requires both good glycaemic control and timely detection and treatment (with photocoagulation) of early-stage, sight-threatening retinal changes. However, there are insufficient numbers of ophthalmologists (about 1 per 1000000 population) or opticians in the region to perform opportunistic screening for diabetic eye disease. ${ }^{159,160}$ Non-physician cadres, such as ophthalmic clinical officers, receive relatively little training in retinal disease, and eye services are overwhelmed by other conditions. ${ }^{161}$ Use of mobile digital photography with telemedicine links is a potential solution to deliver cost-effective, accessible screening to rural and remote populations and, given that fundus cameras remain prohibitively expensive (cost in the region of US\$15000), validation studies are being done for several portable fundus cameras. ${ }^{162,163}$ A simple risk score could be an attractive alternative to screening by retinal fundus photography to identify those who are more likely to be diagnosed with retinopathy (or any other major complications) for transfer to screening and treatment hubs. However, less than $30 \%$ of countries have treatment facilities for retinal photocoagulation. ${ }^{83}$

The prognosis of diabetic nephropathy in populations in HICs has greatly improved over recent decades because of primary prevention (good glucose and blood pressure control) and secondary prevention (regular screening for proteinuria and treatment with an angiotensin-converting enzyme inhibitor after the onset of proteinuria). Furthermore, dialysis and renal transplantation have greatly extended the prognosis of patients with end-stage disease. ${ }^{153}$ Unfortunately, many patients with diabetic nephropathy in sub-Saharan Africa might not have access to such treatments and often progress to end-stage renal disease. ${ }^{100,164}$ Additionally, many parts of sub-Saharan Africa have no nephrologists at all (Kenya has one per 2 million population and South Africa has just over one per million), ${ }^{101}$ and dialysis is unaffordable to many patients. ${ }^{104}$ See the appendix 1 for examples of successful initiatives in sub-Saharan Africa for diabetic retinopathy and diabetic foot disease.

\section{Screening and prevention of type 2 diabetes}

The Commission's consensus is that the best way to manage the diabetes epidemic facing sub-Saharan Africa is to prevent the change in dietary habits and decline in physical activity leading to overweight and obesity that are pervasive across the region. The social, economic, cultural, and political elements that are needed to ensure prevention of these changes are far beyond the remit of this Commission, however, and readers are referred to other extensive literature on this subject-for example, as summarised in The Lancet Physical Activity Series. ${ }^{165}$

Evidence indicates that screening for diabetes in HICs is not beneficial in terms of effect on long-term outcomes. ${ }^{166}$ Additionally, no evidence from LMICs suggests that screening would be a valuable approach to successfully identify and manage people with diabetes or hypertension..$^{167}$ Nevertheless, given the huge number of people in sub-Saharan Africa who have diabetes and go undiagnosed, many academics and policy makers maintain that targeted screening should be done to enable earlier identification and treatment. ${ }^{168}$ Whether such targeted screening will work in practice in LMICs, the risk scores that would be effective for selecting patients to screen, what level of health system infrastructure is needed to enable it, and the cost-to-benefits ratios of instigating such screening are currently unknown. Research is urgently needed to answer these questions before putting in place potentially costly screening programmes. It has also been suggested that platforms for detection of communicable diseases could be co-opted to screen for diabetes and cardiovascular risk factors. Again, however, there is no evidence that such platforms would be a cost-effective method for improving outcomes, and research in this area is needed..$^{52}$

\section{The next steps}

The factors limiting access to prevention and management options for diabetes, its associated cardiovascular risk factors, and long-term complications in sub-Saharan Africa are similar. These factors are poor understanding of diabetes and its complications among health-care professionals and patients; delays in seeking medical attention and in patient referral for specialist care; poor control of glycaemia and other risk factors; inability of patients to afford treatment or transport to attend treatment facilities; and, in some cases, a preference by patients for alternative traditional therapies.

Thus far, we have illustrated the difficulties in provision of good care for patients with diabetes in sub-Saharan Africa. We have also given some examples of successful strategies in HICs, although whether these strategies are transferable to sub-Saharan Africa requires further research. Additionally, diabetes affects multiple physiological systems and interacts with many other diseases to increase the risk of adverse outcomes for a patient. Therefore, a broad-based, health-systemimprovement strategy will clearly be central to improving outcomes in diabetes. In the following sections of the report, we move from clinical considerations to considering the health-system approaches that are necessary to support clinical aims.

\section{Health-system responses to diabetes in sub-Saharan Africa}

Although health systems are crucial in a successful response to diabetes, most research on diabetes in 
sub-Saharan Africa has focused on epidemiology and clinical presentation, with a few studies ${ }^{83,169}$ exploring health systems, although these were limited in scale and scope. We reviewed published studies and analyses of surveys to explore health-system responses to the increasing burden of diabetes in sub-Saharan Africa. We used an established health-systems framework to guide our analysis, and we systematically examined the response to diabetes with regard to the key healthsystem functions of organisation and governance, financing, resource management, and service delivery. ${ }^{170}$

\section{Organisation and governance of diabetes in health systems in sub-Saharan Africa}

The state capacity, organisational and governance structures, and institutional strength of health systems vary across sub-Saharan Africa. In 2010, 42 countries in the WHO African region reported having a unit or department within their ministries of health with responsibility for NCDs, ${ }^{171}$ but just seven countries had a national operational policy, strategy, or plan for diabetes. This situation has ostensibly improved, and, in 2015, the WHO report ${ }^{83}$ on assessing national capacity to address and respond to NCDs-where $35(75 \%)$ of 47 countries in the WHO African region responded to the survey-stated that $100 \%$ of countries in the WHO African region that responded to the survey reported having a unit, branch, or department in their ministry of health that was responsible for NCDs. Additionally, $72 \%$ of countries had an operational policy, strategy, or action plan that integrated NCDs and their risk factors. However, looking specifically at subSaharan Africa, ${ }^{171,172}$ only 20 countries reported having an operational policy, strategy, or action plan for diabetes (nine countries did not and the remainder did not respond).

In 2010, the availability and the stage of implementation of guidelines, protocols, or standards for diabetes management varied across countries. Just four countries in the WHO African region that responded to the WHO report ${ }^{83}$ had guidelines, protocols, or standards that were fully implemented. Although data are not available specifically for sub-Saharan Africa, in 2015, globally, $75 \%$ of countries reported guidelines for dealing with diabetes, ${ }^{83}$ so the situation has probably improved in the region since 2010 .

\section{Financing of health care in sub-Saharan Africa}

In 2014, total health expenditure in sub-Saharan Africa as a proportion of GDP averaged $5 \cdot 5 \%$ (ranging from $6.4 \%$ in the 23 LICs, $6.0 \%$ in the three countries of lowermiddle income, $5.4 \%$ in the five countries of uppermiddle income, and $3 \cdot 3 \%$ in one HIC). ${ }^{7} 12$ countries were below the Chatham House recommendation of $5 \%$ of GDP spent on health. ${ }^{173}$ In the same year, average public spending on health accounted for $42.6 \%$ of total health expenditure. ${ }^{7,12}$ External funding ranged from
64.9\% in The Gambia to $0.3 \%$ in Equatorial Guinea, with an average of $11 \cdot 2 \%$.

In 2001, African nations adopted the Abuja Declaration, pledging to allocate at least $15 \%$ of their national annual budgets to health spending. ${ }^{174}$ Yet, by 2013, only seven countries in sub-Saharan AfricaCentral African Republic, Ethiopia, Malawi, Rwanda, Swaziland, Togo, and Uganda-had reached that target. ${ }^{12}$ In 2014, the average out-of-pocket expenditure as a percentage of total expenditure on health was $34.5 \%$, ranging from $73.5 \%$ in Sierra Leone to $2 \cdot 3 \%$ in Seychelles. ${ }^{7,12,172}$ In seven countries, out-ofpocket expenditure comprised more than $50 \%$ of the total health expenditure (down from 12 countries in 2010). Although unknown, we assume out-of-pocket expenditures to be high for diabetes and often prohibitive, producing financial barriers to access and leading many individuals with diabetes to not seek care (and thus avoiding short-term treatment costs but potentially accumulating larger health deficits, leading to even higher long-term direct costs through more severe sequelae due to target organ damage, such as amputations, blindness, stroke, or kidney failure, in addition to decreased lifespan). Comorbidities and sequelae in many instances result in catastrophic or impoverishing health-care expenditures, sinking many patients and their families beneath the poverty line. For example, in a multicountry study ${ }^{175}$ that included Tanzania, India, China, and Argentina, catastrophic health spending related to cardiovascular events was reported in $92 \%$ of low-income population groups, with distress financing in 4-12\% of low-income groups.

Low levels of public funding, low income levels, and high out-of-pocket expenditures have adversely affected the uptake and provision of care for patients with diabetes, increasing the likelihood of long-term complications. For example, in Malawi in 2012, families spent $22 \%$ of their monthly per-capita budget on out-ofpocket expenditures related to NCDs. ${ }^{176,177}$ For patients with type 1 diabetes, high out-of-pocket expenditures and the unaffordability of care have grave consequences; these patients have a high mortality because regular insulin injections are not always affordable..$^{26,149}$

To increase health financing for NCDs, several countries, such as Cameroon, Botswana, and Seychelles, have introduced earmarked taxation to influence health behaviours, with revenues channelled to health promotion activities. Others have launched reforms to increase public funding for health systems and to achieve universal health coverage, but large informal sectors hinder effective tax collection to invest in health systems. Against this backdrop and the competing demands of a high burden of infectious diseases and other diseases associated with poverty, it is highly unlikely that health systems in sub-Saharan Africa will have adequate financing to deal with an epidemic of diabetes and its consequences. 


\section{Resource management in health systems for tackling diabetes in sub-Saharan Africa}

Sub-Saharan Africa has an acute shortage of health-care professionals; the WHO African Region accounts for $25 \%$ of the current global health workforce shortage, expected to rise to $34 \%$ by 2035 as a result of population growth in Africa. ${ }^{178}$ The shortage of health workers, exacerbated by emigration, ${ }^{179}$ has constrained achievement of the Millennium Development Goals in subSaharan Africa. ${ }^{180}$

There is shortage of medical graduates (more than half of the countries in sub-Saharan Africa have only one medical school, and 11 countries have no medical school ${ }^{178,199}$ ) and nurses, whose level of training and skills vary greatly across countries ${ }^{181}$ More than half of countries in sub-Saharan Africa have a category of non-physician clinician (providers who complete an average of about 3 years of clinical training after secondary education), ${ }^{181}$ and many countries, such as Ethiopia and Malawi, have successfully used community health workers to scale up HIV, tuberculosis, malaria, and other essential services. ${ }^{182,283}$ These strategies should be considered for use in managing

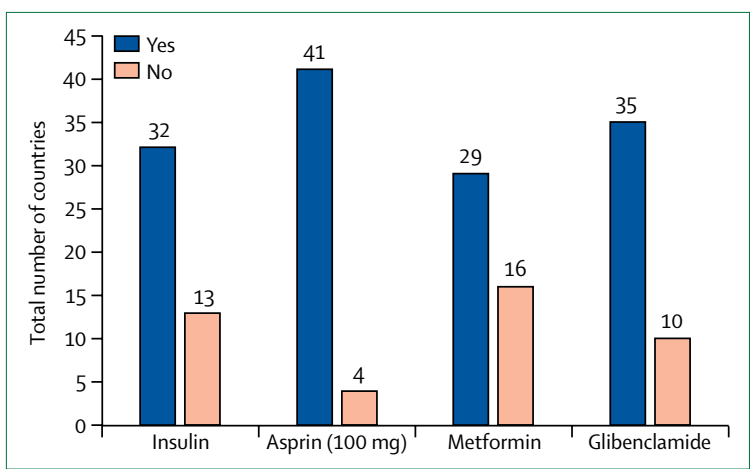

Figure 3: General availability of diabetes medicines in the public health sector in $\mathbf{4 5}$ sub-Saharan African countries

Data are from the WHO Global Health Observatory data repository on

non-communicable-disease-related medicines and are for 2010. Data by country can be obtained from reference 172

\begin{tabular}{|llll|}
\hline & Insulin (\%) & Metformin (\%) & Glibenclamide (\%) \\
\hline Hospital & $48 \%(28-100)$ & $49 \%(21-87)$ & $65 \%(39-94)$ \\
\hline Health centre & $17 \%(0-95)$ & $32 \%(8-64)$ & $31 \%(11-100)$ \\
\hline Primary health centre & $12 \%(1-34)$ & $31 \%(2-65)$ & $48 \%(4-68)$ \\
Dispensary & $13 \%(0-24)$ & $13 \%(0-48)$ & $13 \%(0-43)$ \\
Urban & $7 \%(4-43)$ & $19 \%(6-75)$ & $30 \%(8-71)$ \\
Rural & $12 \%(2-51)$ & $23 \%(1-42)$ & $28 \%(3-72)$ \\
\hline Public & $3 \%(2-35)$ & $7 \%(1-42)$ & $16 \%(3-78)$ \\
\hline Private & $11 \%(5-46)$ & $33 \%(4-86)$ & $39 \%(7-85)$ \\
Overall & $13 \%(3-39)$ & $22 \%(2-57)$ & $32 \%(5-70)$
\end{tabular}

Data are median (range) and are from WHO Service Availability and Readiness Assessment surveys. ${ }^{184}$ Included countries are Benin (2013), Burkina Faso (2012), Democratic Republic of the Congo (2014), Kenya (2013), Mauritania (2013), Sierra Leone (2012), Tanzania (2012), Uganda (2013), and Zambia (2010).

Table 4: Availability of insulin, metformin, and glibenclamide in nine sub-Saharan countries diabetes and other cardiovascular disease risk factors.

The scarcity of human resources affects the capacity of health systems, and their readiness to manage diabetes is revealed by examining WHO Service Availability and Readiness Assessment (SARA) ${ }^{184}$ surveys for ten countries in sub-Saharan Africa (Benin 2013, Burkina Faso 2012 and 2014, Democratic Republic of the Congo 2014, Kenya 2013, Mauritania 2013, Togo 2012, Uganda 2013, Tanzania 2012, Sierra Leone 2012, and Zambia 2010). WHO SARA surveys are designed to assess the national capacity of health systems in countries and can be applied to assess prevention and control of NCDs by measuring the availability of diagnostic tools, essential medications, and trained staff at the health-care-facility level by use of tracer conditions, such as diabetes and cardiovascular disease. ${ }^{184}$ The methodology for the surveys is described elsewhere. ${ }^{184}$ Findings from the country-level SARA reports indicate that major gaps exist in front-line service delivery. In the surveyed countries, less than half of facilities offered diabetes management. Of the facilities not offering diabetes services at the time of the survey, just $40-60 \%$ demonstrated service readiness (table 3 ).

With the exception of Uganda, only about a third of facilities offering diabetes services had guidelines for treatment, and one-third or fewer had at least one diabetes-trained member of staff. The discrepancy between the number of trained staff and availability of diagnostic supplies and drugs is concerning because it is not clear how supplies and drugs are being used in the absence of staff with formal training in diabetes care. The availability of blood glucose testing ranged from $14 \%$ in Burkina Faso (similar to what Beran and colleagues $^{185}$ found in Mali and Mozambique) to $80 \%$ in Uganda, although only $31 \%$ of Uganda sites reported the availability of diabetes-trained staff.

SARA survey data add to the findings of a literature review (see below) to reveal the consequences of underfunded and weak health systems and years of suboptimal investments in human resources, ${ }^{186}$ which have led to large resource gaps for diabetes care in sub-Saharan Africa.

\section{Availability and access to medicines for diabetes in sub-Saharan Africa}

According to WHO, essential medicines for diabetes (metformin, glibenclamide, and insulin) were not available in all sub-Saharan African countries in 2010 (figure 3): of the 45 countries surveyed, metformin was available in 29 countries; glibenclamide was available in 35 countries; insulin was available in 32 countries; and aspirin, used for primary prevention of cardiovascular disease in patients with diabetes, was available in 41 countries that responded. ${ }^{172}$ In 2015, however, WHO found that, of the countries in the WHO African region, 51\% had availability of metformin, $40 \%$ had availability of insulin, and $71 \%$ had availability of aspirin in the public sector. ${ }^{83}$

The WHO Global Action Plan for the Prevention and Control of NCDs 2013-20 has a target of $80 \%$ availability 
for affordable basic technologies and essential medicines, including generic drugs, required to treat major NCDs in both public and private facilities. ${ }^{187}$ Although reaching this target is essential if sub-Saharan African countries are to meet the target of a $25 \%$ relative reduction in premature mortality from NCDs by 2025 , studies reveal access challenges due to a lack of availability and affordability of medications. For example, studies reveal 75\% median availability (actual stocking) of insulin in the public sector (five countries) and $46 \%$ in the private sector (six countries), ${ }^{149}$ and challenges associated with the availability and affordability of oral medicines..$^{139,188-190}$ In 2004-13, only eight $(16 \cdot 7 \%)$ countries in sub-Saharan Africa bought insulin every year and $14(29 \cdot 2 \%)$ countries did not buy insulin at all during this period. ${ }^{191}$

WHO SARA survey reports from $2010-13$ on the nationwide availability of insulin, metformin, and glibenclamide for nine sub-Saharan African countries showed the median availability of insulin to be $13 \%$ (range 3-39; table 4). Insulin availability was generally decreased at lower levels of the health system (ie, primary care), with availability of $12 \%(2-51)$ in rural areas and $7 \%(4-43)$ in urban areas, and 11\% (5-46) in the private sector and $3 \%(2-35)$ in the public sector. The overall median availability of metformin was $22 \%$ (2-57), which also showed decreased availability at lower levels of the health system and increased availability in rural areas and the private sector. The findings were similar for glibenclamide, although glibenclamide was slightly more available in urban areas than in rural areas (table 4). ${ }^{184}$

Despite these findings, these medications are not expensive. Management Sciences for Health (MSH) provide international reference prices for many medicines. ${ }^{192}$ The prices quoted by $\mathrm{MSH}$ are from tenders of ministries of health and represent medicine prices without any add-on costs at the point of entry to a given country. Analysis of data from 1996 to 2013 suggests that the median price for insulin (a $10 \mathrm{~mL}$, $100 \mathrm{IU} / \mathrm{mL}$ vial) in sub-Saharan Africa (eight countries) was US\$7.15 (range \$1.52-17.58) at constant 2015 prices. ${ }^{193}$ Additionally, the median prices were $\$ 0 \cdot 018$ (\$0.002-3.304) for metformin $(500 \mathrm{mg}$, ten countries); $\$ 0.023(\$ 0.012-0.060)$ for gliclazide $(80 \mathrm{mg}$, four countries), as representative of a cheap sulfonylurea that is suitable for patients older than 60 years; and $\$ 0.004$ (\$0.0004-0.032) for glibenclamide ( $5 \mathrm{mg}, 13$ countries). ${ }^{192}$

The treatment costs, using defined daily dose ${ }^{194}$ are shown in table 5 . These data show that treatment of diabetes with insulin presents a significantly higher cost to individuals than treatment with oral drugs. In addition to purchase price, medicine costs are affected by the cost of delivering medicines and mark-ups along the supply chain. ${ }^{149}$ The prices shown in table 5 do not take into account any mark-ups, such as value-added sales tax, local taxes, international purchasing verification tax, insurance, defence levy, overhead mark-ups, bank fees, fees for import declaration forms and port clearance, importer margin, handling costs, wholesale mark-ups, retail mark-ups, health facility mark-ups, dispensing charges, or other mark-ups within the system. The prices also do not reflect the additional costs of ensuring health-care personnel are adequately trained to prescribe the treatments. Although data for these mark-ups are scarce, particularly for insulin, the additional costs of these taxes and levies for the other medicines have been found to range from $18.4 \%$ to $94.4 \%$ of the final retail price of the drug. ${ }^{193}$

These add-on costs, as well as subsidies within health systems, mean that many factors affect the price and affordability of insulin and that the total cost of insulin therapy can vary from $0.2 \%$ of total GDP in South Africa to $13.4 \%$ in Malawi. ${ }^{193}$ For example, data from four studies done in three African countries at different times suggested that medicine prices increased in Mozambique (2003) and Mali (2004) between the central government purchase price and the price paid by health facilities to recuperate storage and transportation costs. ${ }^{185}$ An increase in prices was not observed in Zambia and Mozambique (2009). In Mozambique and Zambia, the difference between the facility purchase price and the patient purchase price was subsidised, whereas in Mali, there was an additional mark-up, such that there was a $47 \%$ increase between the government purchase price and the patient purchase price.

To present different prices of insulin, we combined different data sources to show the median price (at 2015 prices) at different levels of the health system (figure 4). ${ }^{193}$ These data show that, by comparing the MSH prices with those obtained by different ministries of health, many countries in sub-Saharan Africa were purchasing insulin at the best possible price, although there were some outliers. In some countries, insulin was provided free-of-charge or subsidised to patients in the public sector, whereas, in other countries, the prices were higher but still low compared with prices in the private sector. The prices shown in figure 4 are not affordable to individuals in some countries-for example, at the upper end of the scale, a $10 \mathrm{~mL}$ vial of insulin $(100 \mathrm{IU} / \mathrm{mL})$ cost

\begin{tabular}{|lcccc|}
\hline & $\begin{array}{l}\text { Annual } \\
\text { costs (US\$) }\end{array}$ & $\begin{array}{l}\text { Cost per } \\
\text { day (US\$) }\end{array}$ & $\begin{array}{l}\text { Percentage of income } \\
\text { per day }\end{array}$ & \\
\cline { 3 - 5 } & & & US\$1.90* & US\$3.10† \\
\hline Insulin & 104.40 & 0.29 & $15.1 \%$ & $9.2 \%$ \\
\hline Metformin & 26.94 & 0.07 & $3.9 \%$ & $2.4 \%$ \\
Gliclazide & 16.96 & 0.05 & $2.4 \%$ & $1.5 \%$ \\
Glibenclamide & 3.17 & 0.01 & $0.5 \%$ & $0.3 \%$ \\
\hline
\end{tabular}

Data are median. Data come from Management Sciences for Health and authors' calculations. Costs were calculated using defined daily dose. ${ }^{194}$ *US $\$ 1.90$ is the global poverty line, as of October $2015 .{ }^{4}+$ Used as poverty head count ratio. ${ }^{195}$

Table 5: Treatment costs for diabetes medicines and their percentage of two different daily incomes 
US\$10.88 in 2004 in the public sector in Mali or $\$ 50.57$ in 2015 in the private sector in Ethiopia. ${ }^{193}$

Data from WHO/Health Action International (HAI) ${ }^{190}$ suggest that, in some sub-Saharan African countries, people have to pay between $0 \cdot 9$ and $6 \cdot 7$ days of wages to afford 1 month of their diabetes treatment (figure 5). Affordability is defined by WHO/HAI as the lowest-paid government worker paying only 1 day's wage for treatment. Hence, according to the data shown in figure 5, only glibenclamide in Ethiopia is affordable.

However, in sub-Saharan Africa many individuals do not work in the formal sector, and hence measuring affordability in terms of the wage of the lowest-paid government worker is problematic, especially because most people live on less than $\$ 1 \cdot 90-3 \cdot 10$ per day. ${ }^{4} \mathrm{~A}$ comparison of the annual costs of diabetes medicines (table 5) and the daily costs of the different diabetes treatments, using different poverty thresholds of daily

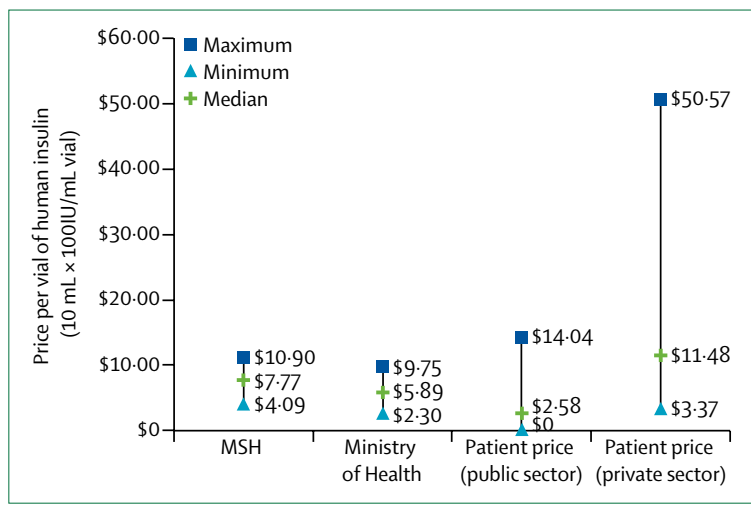

Figure 4: Price per vial of insulin at different levels of the health system Prices (standardised to 2015 US\$) of a vial of human insulin $(10 \mathrm{~mL}, 100 \mathrm{IU} / \mathrm{mL}$ vial) reported by 18 sub-Saharan-African countries. Prices differed in both the public and private sectors depending on whether the vials were purchased from primary care centres, retail pharmacies, or hospitals. Procurement prices obtained by MSH are also shown. Data are from ACCISS Study Insulin Price Profile 2016. ${ }^{193}$ MSH=Management Sciences for Health.

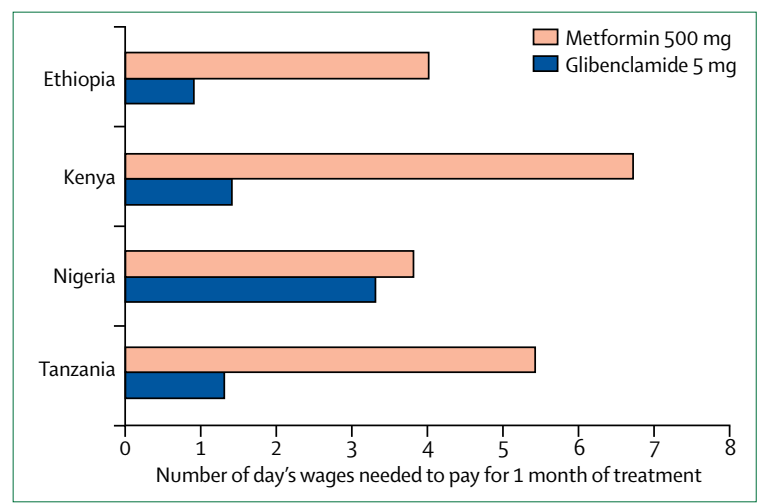

Figure 5: Affordability of oral drugs for diabetes care in day's wages for the lowest-paid government worker in four countries in sub-Saharan Africa Affordability was measured in the number of day's wages that the lowest-paid government worker needed to pay for 1 month of treatment of metformin or glibenclamide in 2004. Data are from the WHO/Health Action International database of medicine prices. ${ }^{190}$ income, shows that daily drug costs (ministry of health purchase prices and not retail price) represent $0 \cdot 5-15 \cdot 1 \%$ of income for someone with diabetes living on $\$ 1 \cdot 90$ per day.

\section{Health service delivery for diabetes in sub-Saharan Africa \\ Analysis of Service Delivery Indicator surveys}

To identify service delivery gaps in diabetes care we analysed data from Service Delivery Indicator (SDI) surveys done in four sub-Saharan African countries by the World Bank, in cooperation with the African Economic Research Consortium and the African Development Bank. ${ }^{138}$ The SDI surveys include data at the level of the health facility on expenditures, provider effort (absence rate, caseload per provider), provider knowledge and ability (diagnostic accuracy, adherence to clinical guidelines, and management of maternal and neonatal complications), and inputs (availability of supplies, equipment, and drugs). Diagnostic accuracy is measured through patient case simulations (vignettes) for seven tracer conditions: malaria with anaemia, diarrhoea with severe dehydration, pneumonia, diabetes, pulmonary tuberculosis, postpartum haemorrhage, and neonatal asphyxia.

The SDI surveys are complementary to and build on surveys (eg, WHO SARA surveys) that focus on the availability of resources and health system readiness for service provision, including for NCDs. Our analysis of SDI surveys broadens and deepens our understanding of service delivery and the quality of diabetes care in subSaharan Africa by providing insights into the knowledge, ability, and effort of providers (technical quality), and the availability of important inputs, such as drugs, equipment, and infrastructure (structural quality; see appendix 1 for additional information and methods).

We investigated the four publicly available SDI surveys: Kenya (2012), Nigeria (2013), Tanzania (2014), and Uganda (2013). When presented with vignettes, less than $53 \%$ of the sampled providers in all countries could accurately diagnose diabetes, with the exception of Kenya where $81 \%$ of providers gave the correct diagnosis (weighted results; figure 6A). Conversely, in all countries except Nigeria, more than $80 \%$ of providers could diagnose tuberculosis, with the proportion as high as $98 \%$ in Kenya and $92 \%$ in Tanzania (figure 6A). Only $4 \%$ of the sample could correctly diagnose all seven conditions. The proportion of providers able to identify all seven conditions was highest in Kenya, where almost $17 \%$ of the surveyed providers correctly diagnosed all conditions presented during the patient simulation.

Although the average clinical guideline scoreindicating the degree of adherence to diabetes clinical guidelines-was less than $30 \%$ in all countries, we observed a wide range of performance scores within countries, with some providers performing all necessary 
tasks in the domains of the score (symptom, patient, history, and physical examination; figure 6B).

More than half of providers who correctly diagnosed diabetes could not prescribe the appropriate treatment, with the exception of Tanzania, where $89 \%$ of the providers who correctly diagnosed diabetes prescribed oral hypoglycaemics (figure 6C). In addition, $77 \%$ of providers in Kenya who correctly diagnosed diabetes indicated that they would refer to higher-level facilities. In other countries, this share ranged from $34 \%$ to $47 \%$ of providers. Whether this pattern of referral is due to a lack of knowledge or confidence in applying the knowledge is not known. However, as the number of diagnosed patients increases, referring the majority to higher-level facilities is unlikely to be a sustainable option.

To investigate the association between provider and facility characteristics and the provider's ability to correctly diagnose diabetes, we did a logistic regression analysis. Table 6 shows a summary of the analytic sample of provider and facility characteristics in these countries. The total sample consisted of 6146 providers, with the largest number of providers coming from Nigeria. With the exception of Tanzania, nurses, midwives, and community health workers represented more than half of the sampled providers. In Tanzania, 75\% of the sample consisted of physicians and medical or clinical officers. In Nigeria, physicians represented only $10 \%$ of the sample, whereas nurses, midwives, and community health workers represented $82 \%$ of the sample (predominantly community health workers). In Kenya, Nigeria, and Uganda, most of the sampled providers were female.

The facility statistics in table 6 show that most providers in all countries were employed at lower-level facilities (eg, dispensaries or health centres) and, except for Tanzania, were primarily located in rural areas. Generally, providers worked at facilities with a high equipment index, suggesting that they had access to a thermometer, adult weighing scale, sphygmomanometer, and stethoscope. In Nigeria and Uganda, however, more than a third of providers did not have access to all four pieces of equipment.

Table 7 shows the logistic regression results of the factors associated with a provider's ability to diagnose diabetes. Female providers had significantly lower odds than male providers of correctly diagnosing diabetes in Kenya (odds ratio $[\mathrm{OR}] 0.58,95 \% \mathrm{CI} 0 \cdot 35-0.98)$ and Nigeria $(0 \cdot 73$, $0 \cdot 61-0 \cdot 86)$, but we cannot explain the reasons behind this difference. Compared with the highest cadre category (physicians, medical officers, and clinical officers), lower cadres had significantly lower odds of diagnosing diabetes in most countries. This result suggests that there is room for improvement in training of lower cadres of health providers in diagnosing diabetes.

It is encouraging that geographical location was not a significant predictor in any of the four countries, with providers in rural areas not having statistically lower odds of diagnosing diabetes than those in urban areas. We did
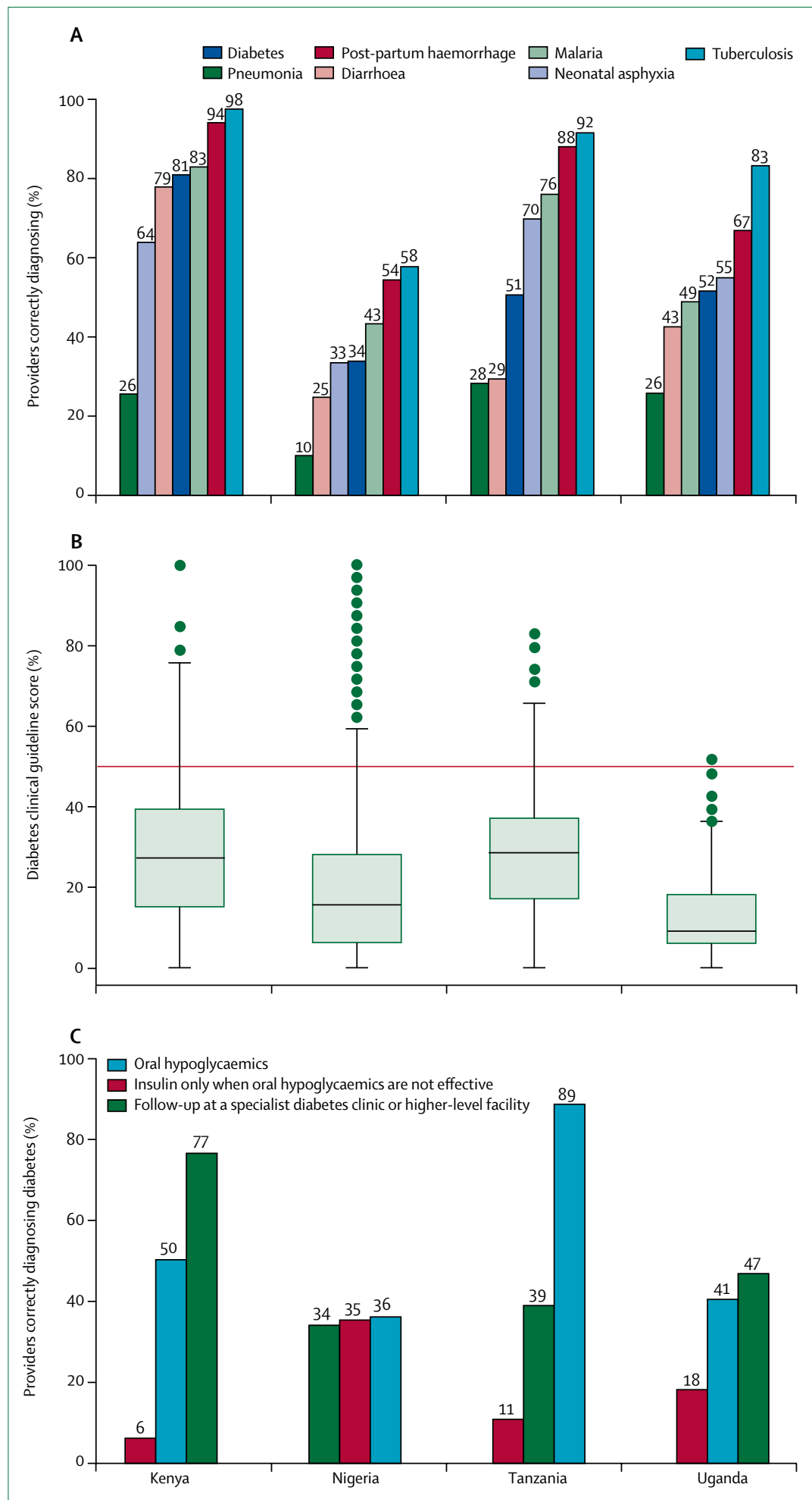

Figure 6: Measures of health service delivery for diabetes in sub-Saharan Africa

Data are from Service Delivery Indicator surveys from four countries: Kenya (2012), Nigeria (2013), Tanzania (2014), and Uganda (2013). The number of providers in each country is shown in table 6. For weighting of the data, see reference 196. (A) Share of providers correctly diagnosing each patient simulation vignette by country. (B) Box plot of clinical guideline scores by country; boxes are shown as median and IQR; the dots show outliers and the red line indicates $50 \%$. (C) Treatment prescribed by providers who correctly diagnosed diabetes. 
not find a statistically significant association between the ability of providers to diagnose diabetes and whether the facility was public in all countries studied. Interestingly, in Kenya and Nigeria, a higher equipment index (table 6) was found to significantly increase the odds of correctly diagnosing diabetes. Although the availability of a glucometer was not generally recorded in the SDI surveys, good availability of the other equipment might be reflective of the availability of a glucometer and hence an increased ability to diagnose diabetes. As mentioned earlier, the variation in the equipment index was quite low, with most providers having access to the basic equipment (particularly in Kenya); thus, the significant ORs suggest that ensuring universal availability of basic equipment could substantially improve diagnostic accuracy.

Table 8 shows the ordinary least squares regression results of the factors associated with higher clinical guideline scores. The dependent variable was the logtransformed clinical guideline score. The results were consistent with the findings for diagnostic accuracy (table 7). Providers in lower cadres were less likely to have high clinical guideline scores than providers in higher cadres. Nurses, midwives, and community health workers were found to have $10-45 \%$ lower clinical guideline scores than physicians and medical and clinical officers. In Nigeria and Tanzania, providers at district hospitals were found to have a $45 \%$ and $42 \%$, respectively, higher clinical guideline score than providers at the

\begin{tabular}{|c|c|c|c|c|}
\hline & $\begin{array}{l}\text { Kenya } \\
(n=526)\end{array}$ & $\begin{array}{l}\text { Nigeria } \\
(n=4388)\end{array}$ & $\begin{array}{l}\text { Tanzania } \\
(n=498)\end{array}$ & $\begin{array}{l}\text { Uganda } \\
(n=684)\end{array}$ \\
\hline \multicolumn{5}{|l|}{ Provider characteristics } \\
\hline \multicolumn{5}{|l|}{ Cadre } \\
\hline Physicians/medical officers & $241(46 \%)$ & $456(10 \%)$ & $371(75 \%)$ & $146(21 \%)$ \\
\hline Nurses/midwives & $22(4 \%)$ & $859(20 \%)$ & $127(25 \%)$ & $520(76 \%)$ \\
\hline Community health workers & $240(46 \%)$ & $2705(62 \%)$ & NA & NA \\
\hline Other & $23(4 \%)$ & $368(8 \%)$ & NA & $18(3 \%)$ \\
\hline Provider is female & $320(61 \%)$ & $2721(62 \%)$ & $199(40 \%)$ & $417(61 \%)$ \\
\hline \multicolumn{5}{|l|}{ Provider performance } \\
\hline Correctly diagnosed diabetes & $431(82 \%)$ & $1492(34 \%)$ & $194(39 \%)$ & $322(47 \%)$ \\
\hline Clinical guideline score* & $0.28(0.16)$ & $0.20(0.17)$ & $0.24(0.14)$ & $0.12(0.09)$ \\
\hline \multicolumn{5}{|l|}{ Facility characteristics } \\
\hline \multicolumn{5}{|l|}{ Facility type } \\
\hline Dispensary & $138(26 \%)$ & $299(7 \%)$ & $306(62 \%)$ & $382(56 \%)$ \\
\hline Health centre & $290(55 \%)$ & $3285(75 \%)$ & $124(25 \%)$ & $284(41 \%)$ \\
\hline District hospital & $98(19 \%)$ & $804(18 \%)$ & $68(14 \%)$ & $18(3 \%)$ \\
\hline Rural & $363(69 \%)$ & $2413(55 \%)$ & $249(50 \%)$ & $534(78 \%)$ \\
\hline Public & $331(63 \%)$ & $4212(96 \%)$ & $339(68 \%)$ & $465(68 \%)$ \\
\hline Equipment index $†$ & $0.97(0.10)$ & $0.77(0.32)$ & $0.95(0.13)$ & $0.79(0.27)$ \\
\hline
\end{tabular}

Data are $\mathrm{n}(\%)$ or mean (SD). The total sample consisted of 6146 providers, of whom 6096 were analysed. NA=not applicable. ${ }^{*}$ The clinical guideline score was calculated as the share of all tasks expected to be done (eg, patient history, symptoms, physical examination) when a patient presents with diabetes symptoms. †The equipment index was calculated as the share of four essential pieces of equipment available and functioning at facilities: sphygmomanometer, thermometer, stethoscope, and weighing scale.

Table 6: Summary statistics of the analytic sample of the Service Delivery Indicator surveys from Kenya (2012), Nigeria (2013), Tanzania (2014), and Uganda (2013) lowest level facilities. The equipment index was also positively associated with the clinical guideline score in Nigeria and Uganda.

The analyses of service delivery indicators suggest that, in the countries studied, there is low readiness across all levels of care and cadres of health professionals in management of diabetes in terms of correct diagnosis, adherence to guidelines, and provision of appropriate treatment. This finding suggests that lower levels of care are unprepared for diabetes diagnosis and treatment, which has implications for development and scaling up of community-based or primary health care-based diabetes management programmes in sub-Saharan Africa.

\section{Implications of health system responsiveness to diabetes in sub-Saharan Africa}

The findings from WHO SARA surveys and World Bank SDI surveys revealed that health systems in sub-Saharan Africa are unprepared for delivery of effective health services for patients with diabetes. However, a comprehensive understanding of how resource and service gaps in health systems affect demand, and how the interaction of supply-side gaps and demand-side dynamics translate into unmet need in sub-Saharan Africa, is constrained by scarce data. In the next section, we analyse surveys of 12 sub-Saharan African countries to examine the nature and extent of unmet need at each crucial stage of the diabetes care process.

\section{Analysis of unmet need and the cascade of care for diabetes in sub-Saharan Africa}

One innovative analytical approach to assess health system performance is the construction of a cascade of care with a tracer condition. Cascade-of-care analysis involves quantitative depiction of the step-wise care for the population affected by a disease of interest, including screening, diagnosis, linkage to treatment programmes, adherence to treatment, and finally achievement and maintenance of control. This analysis depicts the dynamics between demand and health system responses at each step of the care continuum and provides the opportunity to identify areas of unmet need and where attrition in care occurs. ${ }^{11}$

Cascade-of-care analysis has been used to monitor progress towards coverage goals for populations affected by HIV/AIDS. ${ }^{197,198}$ In the USA, 2007-12 data from the National Health and Nutrition Examination Survey (NHANES) have been used to construct a cascade of care for diabetes to show that nearly a third of patients with diabetes are unaware of their diagnosis, and that those who are undiagnosed are less likely than those with a diagnosis to achieve health targets for multiple chronic diseases. ${ }^{199}$

We used individual-level data from population-based surveys done between 2005 and 2013 in 12 sub-Saharan African countries to assess unmet need for care and the care cascade for diabetes. WHO STEPS survey data were 
available for ten countries: Benin, Comoros, Guinea, Kenya, Liberia, Mozambique, Seychelles, Tanzania, Togo, and Uganda. The STEPS survey is a standardised approach to collecting data about cardiovascular NCDs from adults aged 25-64 years in WHO member countries. Briefly, the STEPS surveys include collection of demographic data (step 1); physical measurements such as blood pressure and BMI (step 2); and biochemical measurements, including fasting plasma glucose (step 3). ${ }^{200}$ Further details about the STEPS instrument are provided elsewhere. ${ }^{5}$ Given that a standardised approach is used in all countries, data from the STEPS surveys can be used to compare epidemiology and healthsystem performance across countries.

We supplemented data from STEPS surveys with information from the Demographic and Health Survey for Namibia (2013), ${ }^{201}$ which, similar to STEPS surveys, includes both fasting plasma glucose measurements and self-reported data about access to diagnosis and treatment for diabetes. For South Africa, we used the 2013 South Africa Nutrition and Health Examination Survey (SANHANES), a nationally representative cross-sectional health and nutrition study led by the South African Human Sciences Research Council. ${ }^{202}$ Together, the STEPS, Demographic and Health, and SANHANES surveys consisted of 38311 individuals across 12 countries over the period of 2005-15. How we pooled individual data from different datasets to enable comparability is described in the appendix 1 . In panel 5 we briefly describe the approach used to define diabetes and the method used to construct the care cascade. The methods used to pool data from different surveys, construct the care cascade, and identify unmet need, as well as the limitations of that analysis, are described in detail elsewhere. ${ }^{11}$

The diabetes care cascade across all countries is shown in figure 7. The first step in the cascade is receipt of a diagnostic test, specifically a blood glucose measurement. This initial diagnostic test was associated with the greatest loss to care in all countries, with an average loss to care of $50 \%$ (range 23-81). Among the group who selfreported having received a glucose measurement, the cascade shows that, on average, 13\% (0-26) of the total population with diabetes was then lost to follow-up at the stage of being told about their diagnosis by a health-care provider (figure 7). Among those who reported completing the first two steps in the cascade, an additional $20 \%(5-31)$ of the total population with diabetes was lost to care and follow-up at the stage of receiving advice on lifestyle modification. Finally, a further $6 \%(3-10)$ of the total population with diabetes was lost to care between the stages of receiving advice and receiving any medication, including oral medication or insulin, for diabetes control. Overall, the analysis of the data from the 12 countries showed that the average percentage of the population with diabetes who completed the care cascade was $11 \%$, with a range of $7-33$.

\begin{tabular}{|c|c|c|c|c|}
\hline & Kenya $(n=526)$ & Nigeria $(n=4388)$ & Tanzania $(n=498)$ & Uganda $(n=684)$ \\
\hline \multicolumn{5}{|c|}{ Provider characteristics } \\
\hline \multicolumn{5}{|l|}{ Provider's age } \\
\hline$<30$ years & Ref & Ref & Ref & Ref \\
\hline 30-59 years & $1.73^{*}(1 \cdot 10-2 \cdot 74)$ & $0.77^{*}(0.62-0.95)$ & $1.30(0.66-2.65)$ & $0.83(0.60-1.15)$ \\
\hline$>60$ years & NA & $1.99(0.96-4.13)$ & $1.50(0.52-4.31)$ & NA \\
\hline Provider is female & $0.58 *(0.35-0.98)$ & $0.73+(0.61-0.86)$ & $0.67(0.43-1.05)$ & $0.85(0.57-1.26)$ \\
\hline \multicolumn{5}{|l|}{ Cadre } \\
\hline $\begin{array}{l}\text { Physician/ } \\
\text { medical officer‡ }\end{array}$ & Ref & Ref & Ref & Ref \\
\hline Nurse/midwife & $1.31(0.48-3.59)$ & $0.68 *(0.50-0.94)$ & $0.53^{*}(0.29-0.95)$ & $0.18 \dagger(0.11-0.31)$ \\
\hline $\begin{array}{l}\text { Community } \\
\text { health worker }\end{array}$ & $0.56(0.32-1.01)$ & $0.25+(0.18-0.34)$ & NA & NA \\
\hline Other§ & $0.64(0.20-2 \cdot 01)$ & $0.32 \dagger(0.22-0.47)$ & NA & $0.19 \dagger(0.06-0.64)$ \\
\hline \multicolumn{5}{|c|}{ Facility characteristics } \\
\hline \multicolumn{5}{|l|}{ Facility type } \\
\hline Dispensary & Ref & Ref & Ref & Ref \\
\hline Health centre & $0.80(0.44-1.46)$ & $0.71^{*}(0 \cdot 50-1 \cdot 00)$ & $1.64(0.97-2.76)$ & $2 \cdot 26+(1 \cdot 57-3 \cdot 26)$ \\
\hline District hospital & $1.21(0.48-3.06)$ & $1 \cdot 57^{*}(1.04-2 \cdot 37)$ & $3.08+(1.53-6.19)$ & $2.45(0.78-7.67)$ \\
\hline \multicolumn{5}{|l|}{ Location } \\
\hline Urban & Ref & Ref & Ref & Ref \\
\hline Rural & $0.94(0.52-1 \cdot 70)$ & $0.84(0.70-1.00)$ & $0.92(0.57-1.47)$ & $0.64(0.39-1.03)$ \\
\hline Facility is public & $0.68(0.41-1.15)$ & $1.47(0.92-2.35)$ & $0.81(0.49-1 \cdot 33)$ & $1.32(0.88-1.98)$ \\
\hline Equipment index $\mathbb{T}$ & $8 \cdot 10+(1 \cdot 68-39 \cdot 10)$ & $1.51 \dagger(1.12-2.03)$ & $5 \cdot 70(0 \cdot 78-41 \cdot 77)$ & $2 \cdot 28(0.99-5 \cdot 28)$ \\
\hline \multicolumn{5}{|c|}{$\begin{array}{l}\text { Data analysed were from Service Delivery Indicator surveys from Kenya (2012), Nigeria (2013), Tanzania (2014), } \\
\text { and Uganda (2013). Data are odds ratio ( } 95 \% \mathrm{CI} \text { ). Binary-dependent variable was equal to } 1 \text { if a provider correctly } \\
\text { diagnosed diabetes during the patient simulation vignette, } 0 \text { otherwise. } N A=\text { not applicable. }{ }^{*} p \leq 0 \cdot 05 .+p \leq 0 \cdot 01 \text {. } \ddagger \text { Also } \\
\text { includes clinical officers. SIncludes paraprofessionals and lab technicians. } \text { IEquipment index was calculated as the share } \\
\text { of four essential equipment available and functioning at the facilities: sphygmomanometer, thermometer, } \\
\text { stethoscope, and weighing scale. }\end{array}$} \\
\hline
\end{tabular}

The care cascades for three exemplar countries (Mozambique, Kenya, and South Africa) are shown in the appendix 1 . These three countries were chosen as examples because they represent three very different levels of wealth, as measured by GDP per capita in 2011 international dollars for the year of the survey (Mozambique [2005]: Int1\$735, Kenya [2015]: \$2818, South Africa [2013]: \$12 375).

Our analysis shows unmet need at every step of the diabetes care continuum, which leaves around $50 \%$ of patients with diabetes going undiagnosed and further substantial dropoffs in care throughout the remainder of the cascade. From this analysis, and what is known about unmet need in the region, we surmise that for people diagnosed with or at risk of diabetes, health systems are unable to provide the needed services, with most patients not receiving the necessary advice and medication. Unmet need and suboptimal care mean that patients will probably have delayed presentation to the health system and receive advice and medication late in the care process. Delayed presentation and treatment have adverse effects on health outcomes in diabetes, leading to 


\begin{tabular}{|c|c|c|c|c|}
\hline & $\begin{array}{l}\text { Kenya } \\
(n=526)\end{array}$ & $\begin{array}{l}\text { Nigeria } \\
(n=4438)\end{array}$ & $\begin{array}{l}\text { Tanzania } \\
(\mathrm{n}=498)\end{array}$ & $\begin{array}{l}\text { Uganda } \\
(n=684)\end{array}$ \\
\hline \multicolumn{5}{|l|}{ Provider characteristics } \\
\hline \multicolumn{5}{|l|}{ Provider's age } \\
\hline$<30$ years & Ref & Ref & Ref & Ref \\
\hline $30-59$ years & $-0.06(0.06)$ & $-0.11^{*}(0.05)$ & $0.04(0.10)$ & $0.05(0.07)$ \\
\hline$>60$ years & NA & $-0.07(0.13)$ & $-0.15(0.19)$ & NA \\
\hline Provider is female & $-0.10(0.11)$ & $-0.35+(0.01)$ & $-0.04(0.07)$ & $-0.21 \dagger(0.07)$ \\
\hline \multicolumn{5}{|l|}{ Cadre } \\
\hline Physician/medical officer & Ref & Ref & Ref & Ref \\
\hline Nurse/midwife & $-0.10(0.11)$ & $-0.30+(0.08)$ & $-0.21^{*}(0.08)$ & $-0.45+(0.09)$ \\
\hline Community health worker & $-0.24 \dagger(0.08)$ & $-0.34 \dagger(0.08)$ & NA & NA \\
\hline Others & $-0.35(0.27)$ & $-0.41 \dagger(0.10)$ & NA & $-0.15(0.18)$ \\
\hline \multicolumn{5}{|l|}{ Facility characteristics } \\
\hline \multicolumn{5}{|l|}{ Facility type } \\
\hline Dispensary & Ref & Ref & Ref & Ref \\
\hline Health centre & $0.01(0.10)$ & $0.08(0.09)$ & $0.28+(0.08)$ & $0.29+(0.08)$ \\
\hline District hospital & $-0.07(0.13)$ & $0.45+(0.11)$ & $0.42+(0.12)$ & $0.17(0.21)$ \\
\hline \multicolumn{5}{|l|}{ Location } \\
\hline Urban & Ref & Ref & Ref & Ref \\
\hline Rural & $-0.18^{*}(0.08)$ & $0.04(0.04)$ & $-0.08(0.08)$ & $0.02(0.09)$ \\
\hline Facility is public & $0.10(0.08)$ & $0.03(0.11)$ & $0.00(0.08)$ & $0.02(0.09)$ \\
\hline Equipment index $\boldsymbol{\Phi}$ & $0.59(0.35)$ & $0.19^{*}(0.07)$ & $-0.02(0.25)$ & $0.34^{*}(0.15)$ \\
\hline
\end{tabular}

Data analysed were from Service Delivery Indicator surveys from Kenya (2012), Nigeria (2013), Tanzania (2014), and Uganda (2013). Data are coefficients from ordinary least squares regressions, with the log(clinical guidelines score) as the dependent variable and robust SEs in parentheses. The clinical guideline score was calculated as the share of all tasks expected to be done (eg, patient history, symptoms, physical exam) when a patient presents with diabetes symptoms. $N A=$ not applicable. ${ }^{*} p \leq 0.05$. $\uparrow p \leq 0.01$. $\neq$ Also includes clinical officers. SIncludes paraprofessionals and lab technicians. TEquipment index was calculated as the share of four essential equipment available and functioning at facilities: sphygmomanometer, thermometer, stethoscope, and weighing scale.

Table 8: Ordinary least squares regression results with the log of the clinical guideline score as the dependent variable

difficulties for patients and their families due to ill health and to adverse economic consequences for patients, their families, and their countries.

\section{Economic consequences of diabetes in sub-Saharan Africa}

Economic burden of diabetes to individuals

In addition to ill health and substantial reductions in quality of life, diabetes imposes a non-negligible financial burden on affected individuals, families, and societies. While patients with diabetes face direct costs of illness through medical treatment of the disease and its comorbidities and sequelae, they also experience income losses through reduced productivity and disability, which means inability to work in severe cases.

The relatively high prices for necessary health items, such as blood glucose strips and insulin, impose a considerable financial burden on individuals, as discussed in previous sections of this report. Combined with the substantial reliance of health systems in sub-Saharan Africa on out-of-pocket expenditure, patients with diabetes in the region often have only limited access to adequate and timely treatment, potentially resulting in an increased risk of diabetes-related health complications. Moreover, comorbidities and target organ damage due to diabetes might cause catastrophic health-care expenditures, shifting many patients and their families beneath the poverty line. ${ }^{203}$ Simultaneously, these adverse effects are likely to be perpetuated in the absence of adequate social security systems; families might attempt to offset such catastrophic expenditures by putting children into the workforce, thus cutting short their education and reducing their future prospects of financial wellbeing.

Measurement of the direct economic burden on individuals is complicated by the fact that health expenditure for diabetes-related complications is difficult to quantify because diabetes might not be the only attributable cause. The IDF therefore derives estimates for direct costs of diabetes from each country's total health expenditure, applying age-specific and sex-specific ratios of average heath expenditure to people with and without diabetes. Accordingly, estimated per-patient expenditures for diabetes in 2015 in sub-Saharan Africa ranged between US $\$ 243$ and $\$ 419,,^{25}$ although the validity of these numbers is unknown because cost ratios were based on US data. A 2017 study $^{204}$ that used a similar approach but different cost ratios for LMICs found average per-patient costs of $\$ 580$. Other studies estimated direct costs of diabetes per person each year to be $\$ 138$ in Tanzania in 1989-90, ${ }^{205}$ and $\$ 489$ in Cameroon in 2001. ${ }^{206}$ Although not all of these expenditures are borne by individuals directly through out-of-pocket payments, increased insurance contributions and taxes can serve to further burden individuals.

Indirect costs of illness result from productivity losses of workers during their productive years (because costs of early mortality are not borne by patients themselves, we do not consider this position in this section). These productivity losses comprise absenteeism (sick workers failing to appear for work), presenteeism (unfit workers coming to work but not performing to full capacity), and labour-force dropout (panel 6). Notably, whereas in HICs productivity losses might be partially or fully offset by social security systems (eg, continued pay during sick leave, insurance payments in case of permanent disability), in sub-Saharan Africa productivity losses are likely to fully accrue at the level of the sick individual through foregone wages from formal or informal work and reduced agricultural yield in the case of subsistence farmers.

\section{Economic burden of diabetes to countries}

Evidence about the total economic burden of diabetes to societies in sub-Saharan Africa is scarce. Kirigia and colleagues $^{215}$ estimated that the combined direct and indirect costs amounted to Int $\$ 25 \cdot 51$ billion (2005 purchasing power parity) in 2000 , but they might not have captured the full picture because diabetes-related complications were excluded from their analysis. We therefore based our evaluation of economic burden to countries on a top-down approach used in a study by 
Bommer and colleagues. ${ }^{204}$ Analysing direct health expenditure and indirect costs of diabetes, that study estimated the global economic burden in adults in 2015 to be US\$1.31 trillion, equivalent to $1.8 \%$ of global GDP.

Using prevalence and mortality data from the IDF Diabetes Atlas, ${ }^{25}$ the study ${ }^{204}$ estimated direct costs based on countries' per-capita health expenditure, assuming literature-derived ratios between age-specific and sexspecific treatment costs for people with and without diabetes. These ratios varied between HICs and LMICs, between women and men in HICs, between rural and urban areas in LMICs, and between people with diagnosed versus undiagnosed diabetes, thus extending previous work by the IDF..$^{25}$ Note, however, that no appropriate studies for sub-Saharan Africa could be identified, and thus the applied cost ratios might not fully reflect the situation in the region. Indirect costs were defined as productivity losses due to mortality or disability, as measured by foregone labour earnings. As wage data from sub-Saharan African countries are scarce, labour earnings were proxied by the labour income share in GDP per working age person, as measured in 2015 US\$. A more detailed discussion of the method is provided elsewhere ${ }^{204}$ and in the appendix 1.

Based on the same approach as that used by Bommer and colleagues, ${ }^{204}$ and using national account data from the World Development Indicators database ${ }^{12}$ for 47 countries in sub-Saharan Africa, we estimated the overall costs of diabetes in 2015 for sub-Saharan Africa to be US $\$ 19.45$ billion, or $1.2 \%$ of cumulative GDP of the entire region. About $\$ 8.64$ billion $(44.4 \%$ ) of this burden arose from indirect costs. About $\$ 12 \cdot 10$ billion $(62 \cdot 2 \%)$ arose from southern Africa, mainly from the relatively wealthy South Africa, and only $\$ 1.70$ billion $(8 \cdot 7 \%)$ arose from western Africa. The share of indirect costs by region varied from $23 \cdot 2 \%$ to $49 \cdot 2 \%$ (figure 8 ).

Productivity losses consisted of four components: premature mortality, which amounted to $\$ 7.86$ billion $(91.0 \%)$ of total indirect costs; diabetes-related complications and malaise, leading to workforce dropout, which accounted for $\$ 0.53$ billion $(6 \cdot 2 \%)$ of total indirect costs); sick leave (absenteeism), which accounted for $\$ 0.17$ billion $(1.9 \%)$ of total indirect costs; and decreased productivity while working (presenteeism), which amounted to $\$ 0.07$ billion $(0.9 \%)$ of total indirect costs (figure 9). These estimates provide important benchmarks for the economic value that could be generated through improvements in initiatives to address diabetes risk factors, prevention, and early diagnosis.

An important question for policy makers in subSaharan Africa is how the economic costs of diabetes are going to evolve in the short and medium term. To predict economic costs of diabetes over time, we followed previous projection attempts by the $\operatorname{IDF}^{25}$ by using quinquennial UN Population Prospects data for the years 2015-30 (using the medium variant provided by the UN Population Division), ${ }^{216}$ as well as projected urbanisation rates from the UN. ${ }^{217}$ Both demographic changes and urbanisation rates are likely to be substantial drivers of future costs because an urban sedentary lifestyle is considered an important risk factor for diabetes. ${ }^{218}$ Our projected economic costs also take into consideration real GDP and GDP per-capita growth (extrapolated based on past growth rates obtained from the World Bank). ${ }^{12}$

\section{Panel 5: Constructing the diabetes care cascade}

When constructing the diabetes care cascade, diabetes was defined based on the current WHO and American Diabetes Association diagnostic criteria as a fasting plasma glucose concentration of more than or equal to $7.0 \mathrm{mmol} / \mathrm{L}(126 \mathrm{mg} / \mathrm{dL})$, a $2 \mathrm{~h}$ plasma glucose concentration of more than or equal to $11.1 \mathrm{mmol} / \mathrm{L}(200 \mathrm{mg} / \mathrm{dL})$, or a $\mathrm{HbA}_{\mathrm{lc}}$ measurement of $6.5 \%$ (48 mmol/mol) or higher. ${ }^{136,137}$ This definition represents the goldstandard clinical practice guidelines that are being used internationally. The data had one or more of these measures for each individual surveyed (appendix 1 ).

Those individuals reporting use of medication for diabetes were also classified as having diabetes, irrespective of the biomarker values. Respondents who self-reported a diagnosis of diabetes, but were not on medication and lacked the criteria indicated above, were not classified as diabetic. Additionally, we quantified met need for four different metrics of diabetes care in the population with diabetes: ever having received a blood glucose measurement as a measure of diagnosis (before the STEPS or other survey with which the diagnosis was made); for those who had received a blood glucose measurement, ever having been told about the diagnosis of diabetes as a measure of awareness of diagnosis; receipt of any advice from a health-care provider to lose weight or exercise; and use of either oral medications or insulin for treatment of diabetes.

Using these metrics, we constructed a diabetes care cascade for each of the 12 countries for which data were available. This cascade, created with individual-level data, shows the percentage of the total population with diabetes that self-reported reaching each subsequent step in the care process, conditional on having reached the previous step.

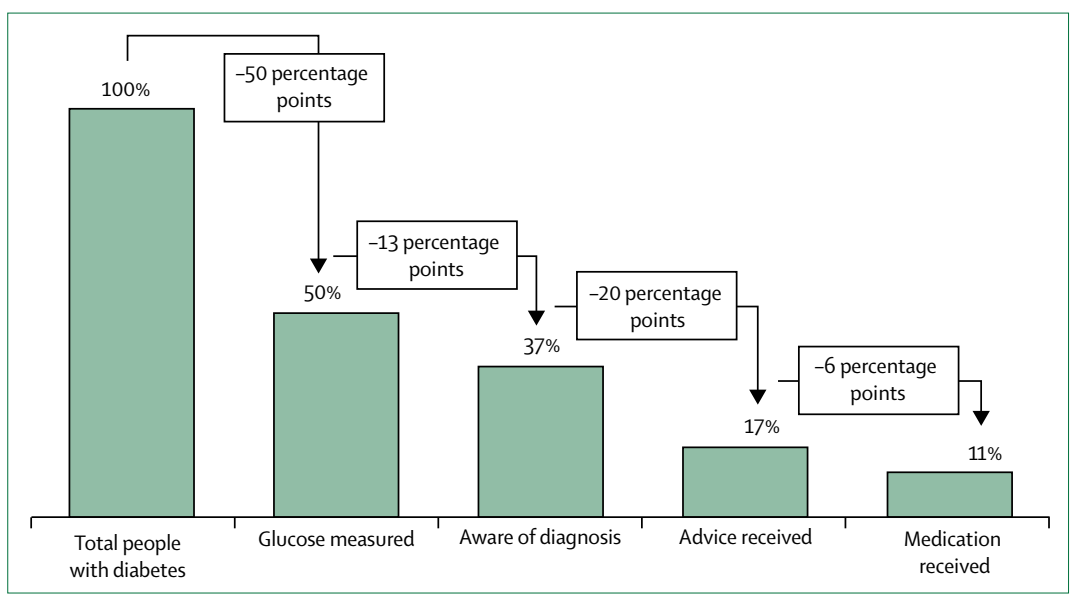

Figure 7: Cascade of care for diabetes based on population survey data from 12 sub-Saharan African countries (2005-15)

This figure depicts the diabetes cascade of care based on data from 10 STEPS surveys (Benin, Comoros, Guinea, Kenya, Liberia, Mozambique, Seychelles, Tanzania, Togo, and Uganda), the Namibia Demographic and Health Survey, and the South Africa Nutrition and Health Examination Survey. All surveys were done during the period from 2005 to 2015. By use of individual-level data from these surveys, we created a diabetes care cascade, which shows the percentage of the total population with diabetes that self-reported reaching each subsequent step in the care process, conditional on having reached the previous step. See Panel 5 on how diabetes was defined. All numbers displayed represent the percentage of the total number of people with diabetes in the sample. 


\section{Panel 6: Labour-market effects of diabetes}

Based on a systematic review and assessment of the available empirical evidence on the labour-market effects of diabetes, a global cost-of-illness study ${ }^{204}$ estimated that the reduction in labour-force participation of individuals with diabetes in high-income countries (HICs) was $12.6 \%$ for men and $25 \cdot 2 \%$ for women. Conversely, in low-income and middle-income countries (LMICs), reduction in labour-force participation ranged from $1 \cdot 1 \%$ to $13 \cdot 2 \%$ for men and from $1.2 \%$ to $17 \cdot 4 \%$ for women. Moreover, in high-income settings, men and women with diabetes who were in the labour force were found to be absent from work for 1.9-4.3 additional days per year, whereas the corresponding numbers in LMICs ranged from 1.9 to 8.6 excess days for men and from 2.8 to 10.2 excess days for women. Finally, productivity losses while at work (presenteeism) in people with diabetes were found to be $0.3 \%$ in HICs and $0.6-1.0 \%$ in LMICs.

The underlying empirical evidence largely draws on data from HICs and upper-middle-income countries. Effects on labourmarket dropout and presenteeism are based on studies from the USA ${ }^{207,208}$ and Mexico. ${ }^{209,210}$ Although the diversity of sources is larger for absenteeism-with studies from the USA ${ }^{211}$ Mexico, ${ }^{210}$ India, ${ }^{212}$ Iran ${ }^{213}$ and Namibia ${ }^{214}$-it is unclear to what extent these labour-market effects accurately capture the situation in sub-Saharan Africa. For instance, the combination of limited capabilities for management of blood sugar levels and a shortage of preventive treatment is likely to lead to high rates of severe complications in the long term hence potentially increasing the rate of labour-force dropout.

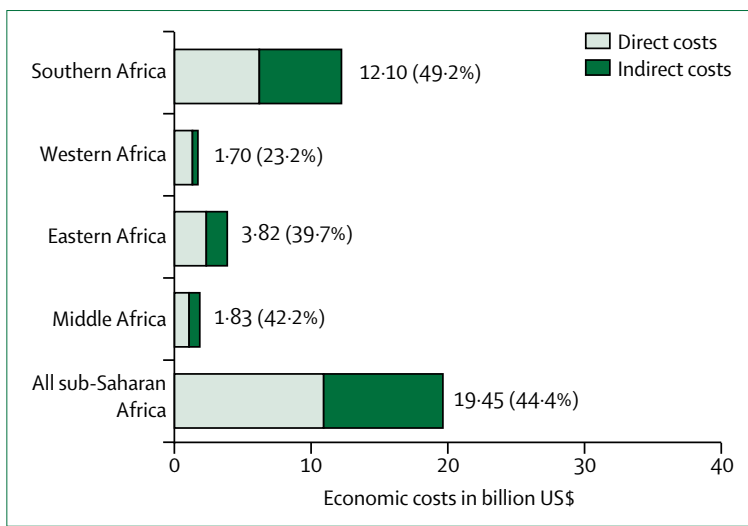

Figure 8: Economic burden of diabetes in sub-Saharan Africa (2015) Numbers in parentheses are percentages of indirect costs in total costs. Data are from the World Development Indicators database ${ }^{12}$ and are for 47 countries in sub-Saharan Africa. Estimates for southern Africa include Botswana, Lesotho, Namibia, South Africa, and Swaziland. Estimates for western Africa include Benin, Burkina Faso, Cape Verde, Côte d'Ivoire, The Gambia, Ghana, Guinea, Guinea-Bissau, Liberia, Mali, Mauritania, Niger, Nigeria, Senegal, Sierra Leone, and Togo. Estimates for eastern Africa include Burundi, Comoros, Eritrea, Ethiopia, Kenya, Madagascar, Malawi, Mauritius, Mozambique, Rwanda, Seychelles, South Sudan, Sudan, Tanzania, Uganda, Zambia, and Zimbabwe. Estimates for middle Africa include Angola, Cameroon, Central African Republic, Chad, Democratic Republic of the Congo, Republic of the Congo,

Equatorial Guinea, Gabon, and São Tomé and Príncipe.
We considered three scenarios for the evolution of agegroup-specific and sex-specific diabetes prevalence and mortality. First, we used the optimistic assumption that age-group-specific and sex-specific diabetes mortality and prevalence stay constant over time (scenario A). Second, we let the age-group-specific and sex-specific diabetes prevalence and mortality increase, depending on a country's income-group classification according to the World Bank and adult diabetes prevalence in 2015, as shown in table 9 (scenario B). The rationale for this approach was the assumption that middle-income countries will increasingly adopt western sedentary lifestyles and consumption patterns. This trend is likely to be less pronounced in LICs where restricted household budgets constrain rapid changes in consumption patterns. Third, in scenario C, we doubled all growth rates from scenario B. Thus, our projections cover very optimistic to very pessimistic outlooks.

In addition to changes in prevalence and mortality, the growth of direct costs depends in part on remuneration of health personnel; Organisation for Economic Cooperation and Development data suggested that compensation of health workers accounted for up to $57 \%$ of total health expenditure during the past decade, and we assumed sub-Saharan African health sectors to be particularly labour intensive. ${ }^{219}$ Similarly, the increase in indirect costs depends on the evolution of average annual wages. For all projection scenarios, we assumed that both average wages and the remuneration of health personnel grow at the same rate as real GDP per capita.

Our estimates suggest that the economic costs of diabetes for sub-Saharan Africa will increase from US $\$ 19.45$ billion (1.2\% of GDP) in 2015 to $\$ 35.33$ billion $(1 \cdot 1 \%$ of GDP) in 2030 , according to scenario A; to $\$ 47.33$ billion (1.4\% of GDP) in 2030, according to scenario B; and to $\$ 59.32$ billion (1.8\% of GDP) in 2030 , according to scenario C (measured in 2015 prices; figure 10).

While all projection scenarios place southern Africa on top, with an increase from US\$12.10 billion in 2015 to $\$ 17 \cdot 15-\$ 29 \cdot 20$ billion in 2030 depending on the scenario, we also predict substantial growth in absolute costs in eastern Africa (from \$3.82 billion in 2015 to up to $\$ 16 \cdot 21$ billion in 2030 for scenario C; figure 10). Relative to GDP, southern Africa is again predicted to bear the largest economic burden in all projection scenarios (from 3.5\% in 2015 to between 3.4\% for scenario A and $5.8 \%$ for scenario C in 2030; figure 10 ).

Despite uncertainties about the future evolution of diabetes prevalence and diabetes-related mortality, and future GDP growth, the numbers are alarming. The high direct and indirect economic burden sub-Saharan Africa is predicted to face creates a strong incentive for policy makers to increase efforts to prevent diabetes and reduce diabetes-related complications and premature mortality. However, as the analysis in this report shows, health systems in sub-Saharan African countries are illprepared to effectively manage diabetes; the existing 
health-systems response is weak, the care provided is suboptimal, and the unmet need is very large. If diabetes is effectively managed, its future health and economic burden could be substantially reduced. The following sections therefore point to potential health policies that might help sub-Saharan African countries to better cope with the challenges imposed by diabetes, and discuss the potential benefits that could be realised if diabetes in sub-Saharan Africa was to be managed according to international guidelines and evidence.

\section{Benefits of scaling up diabetes interventions in sub-Saharan Africa}

For people with diabetes, the three principal coexisting risk factors leading to morbidity and mortality are high blood pressure, disordered lipid profile, and poor glycaemic control; an aim of good diabetes care is to prevent their long-term complications. Although all three risk factors have been associated with macrovascular and microvascular complications, substantial differences exist in their relative effects, in terms of the complexity of their treatment and monitoring regimens, their therapeutic window, and the costs of therapy. Important interactions exist between these risk factors for both macrovascular (coronary heart disease, stroke) and microvascular (retinal, renal, neuropathic) complications of diabetes, ${ }^{220,221}$ with treatment guidelines emphasising the importance of addressing all three. ${ }^{222}$

For both blood pressure and lipid therapies, there has been a move towards targeting treatment to individuals at increased levels of risk, rather than according to levels of blood pressure or lipids, with the understanding that different individuals might experience different benefits and risks from therapy depending on their comorbid conditions. For example, people with previous myocardial infarction or stroke might benefit from initiation of treatment at lower levels of blood pressure or LDL-cholesterol than those who have not had a previous cardiovascular event. The risk-based approach to treating blood pressure, termed benefit-based tailored treatment (BTT), has been shown to be more effective and less costly than treating blood pressure to target levels (the so-called treat-to-target [TTT] strategy). ${ }^{223}$ Additionally, when considering blood pressure, lipid, and glycaemic control in patients with diabetes, in countries with poor insulin availability (eg, many LMICs), BTT was shown to be more clinically effective and cost-effective than TTT for preventing microvascular and macrovascular complications. When insulin was available, the BTT strategy was no longer superior to the TTT strategy for preventing microvascular disease. ${ }^{152}$

Using the same model as used in our previous study, ${ }^{152}$ we tested, with a microsimulation model, whether a BTT approach compared with the status quo (ie, currently received care) or a TTT approach compared with the status quo would be beneficial to people with diabetes in countries in sub-Saharan Africa for overall management of diabetes risk factors. A summary of the methods is shown in panel 7. We found that, from a population perspective, a BTT strategy would be more effective and cost-effective than a TTT strategy (table 10). Although a similar proportion of people with diabetes would typically be recommended treatment of any kind (for example, a mean of $86.4 \%$ under the TTT strategy vs $88.4 \%$ under the BTT strategy in Malawi; table 10), those typically treated with the BTT strategy would be treated more intensively (for example, 4.5 vs 3.5 medications per person in Malawi; table 10).

Compared with the TTT strategy, the BTT strategy would recommend significantly more adults with diabetes to receive blood-pressure-lowering drugs and would non-significantly increase the number of patients treated with statins and glucose-lowering therapies (table 10). Additionally, the BTT strategy was estimated to avert two-to-four times as many macrovascular events (myocardial infarction and stroke) as the TTT strategy over a period of 10 years, although the number of microvascular events did not significantly differ between the two strategies.

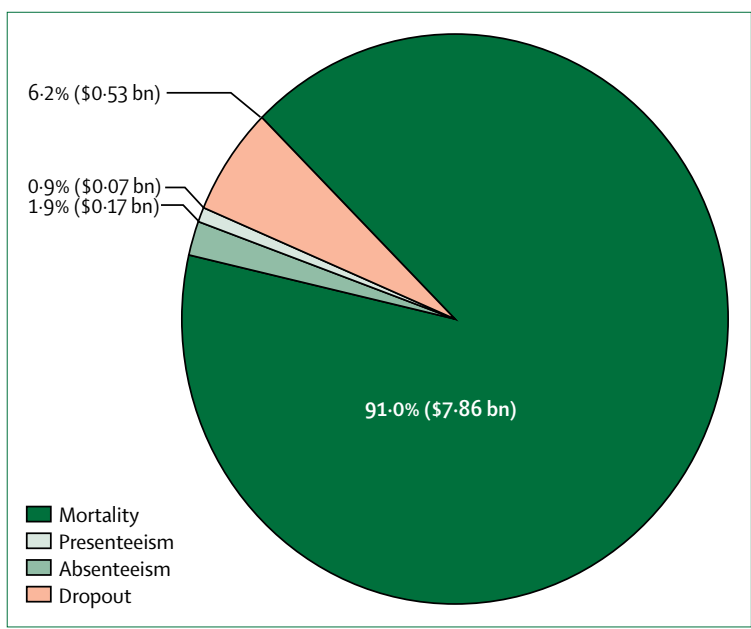

Figure 9: Distribution of indirect costs of diabetes in sub-Saharan Africa (2015) Data are authors' estimates. Productivity losses consisted of four components: premature mortality, reduced labour-force participation due to diabetes-related complications and malaise (dropout), increased likelihood of taking sick leave (absenteeism), and decreased productivity while working (presenteeism).

\begin{tabular}{|llll|}
\hline & Prevalence $\leq 3 \%$ & $\begin{array}{l}\text { Prevalence }>3 \% \\
\text { to } \leq 7 \cdot 5 \%\end{array}$ & Prevalence $>7 \cdot 5 \%$ \\
\hline Low-income countries & $0 \% / 35 \% / 70 \%$ & $0 \% / 20 \% / 40 \%$ & $0 \% / 5 \% / 10 \%$ \\
Middle-income countries & $0 \% / 50 \% / 100 \%$ & $0 \% / 35 \% / 70 \%$ & $0 \% / 17 \cdot 5 \% / 35 \%$ \\
High-income countries & $0 \% / 25 \% / 50 \%$ & $0 \% / 10 \% / 20 \%$ & $0 \% / 2 \cdot 5 \% / 5 \%$ \\
\hline
\end{tabular}

Data are the assumed growth in age-group-specific and sex-specific prevalence and mortality from 2015 to 2030 for scenarios A/B/C, depending on 2015 adult prevalence and income classification. Data for 2015 come from the International Diabetes Federation Diabetes Atlas. ${ }^{25}$ Growth rates for high-income countries with low or medium diabetes prevalence are only provided for reference, since no country in sub-Saharan Africa fell into this category.

Table 9: Summary of scenarios for the evolution of age-group-specific and sex-specific prevalence and mortality by income group and 2015 adult prevalence for countries in sub-Saharan Africa 


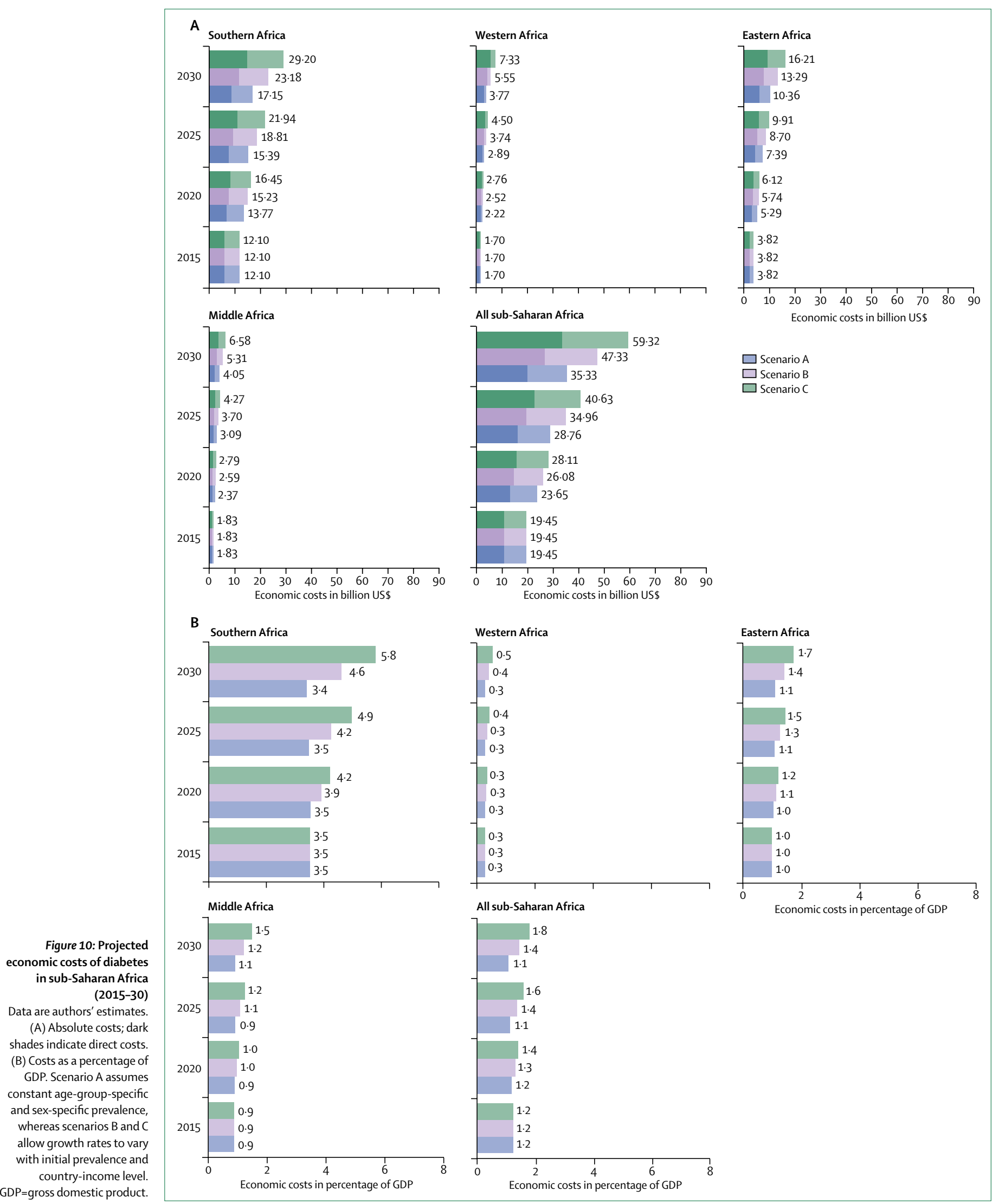


Panel 7: Benefits of managing diabetes using a benefit-based tailored treatment or treat-to-target strategy in sub-Saharan Africa

We constructed a microsimulation model, which simulates individuals with diabetes by sampling from correlated probability distributions describing the relationships between demographics and risk factor values in the populations of interest. Individual demographics included age and sex, with population sizes taken from the UN Population Division's projections for 2015-25, and the subset of the population expected to be born or already alive with diabetes during this period based on International Diabetes Federation estimates for diabetes prevalence and trends for the age group of 30-70 years. ${ }^{12}$ The microsimulation model (method published in detail elsewhere ${ }^{152}$ and described in the appendix 1 ) enabled us to compare the approaches to reducing the risk of major macrovascular (myocardial infarction, stroke) and microvascular (neuropathy, retinopathy, and end-stage renal disease) complications of diabetes in two populations aged 30-70 years in sub-Saharan Africa: one from Malawi ( $n=35730)$, who are participants in the Karonga Prevention Study, ${ }^{224}$ and the other from combined datasets from Ghana and South Africa ( $n=3938$ and 2352,

respectively), representing sub-Saharan $A$ frica (see appendix 1 for methods).

The methods and limitations have been described in detail elsewhere. ${ }^{152,223}$ Briefly, two alternative management approaches were compared. The first was a treat-to-target (TTT) strategy involving titration of blood pressure medication, statins, and glucose-lowering drugs to predefined targets. Thus, the TTT strategy involved treating individuals with blood pressure medication until they achieved a blood pressure of less than $130 / 80 \mathrm{~mm} \mathrm{Hg} ;{ }^{225}$ with a statin until they achieved an LDL concentration of less than $2.59 \mathrm{mmol} / \mathrm{L}(100 \mathrm{mg} / \mathrm{dL}){ }_{i}^{226}$ and with metformin and sulfonylureas (and, if needed, substituting the sulfonylurea with insulin) until they achieved an $\mathrm{HbA}_{1 c}$ level of less than $7 \% .{ }^{136,137}$

The second strategy was a benefit-based tailored treatment (BTT) strategy, which involved treating individuals at high risk of macrovascular complications with blood pressure medication and statins, and those at high risk of microvascular complications with glucose-lowering agents, until they achieved low risk levels. Specifically, the BTT strategy involved treating individuals with a 10-year combined risk (assessed using UKPDS outcomes model 2-see appendix 1 for further details) of myocardial infarction and stroke of more than $10 \%$ with antihypertensive drugs and a statin until their risk was lowered to below the $10 \%$ threshold (provided blood pressure remained $>110 / 55 \mathrm{~mm} \mathrm{Hg}$ for safety). Additionally, the strategy involved treating those with a lifetime risk of the three major microvascular complications (blindness, end-stage renal disease, and amputation secondary to neuropathic ulcer) of more than $4 \%$ with metformin and sulfonylureas (and, if needed, substituting the sulfonylurea with insulin) until lifetime microvascular risk was below $4 \%$ (provided fasting blood glucose remained $>3.33 \mathrm{mmol} / \mathrm{L}[60 \mathrm{mg} / \mathrm{dL}]$ for safety).

We compared each method with the current situation in each individual country and then compared the average cost-effectiveness ratios of the BTT and TTT strategies. We used WHO guidelines ${ }^{227}$ to choose blood pressure and statin medications and the Yale Diabetes Center Guidelines ${ }^{228}$ for dose-escalation algorithms for metformin, sulfonylureas, and insulin.

Cases averted, disability-adjusted life-years (DALYs) saved (based on disability weight values estimated by the Global Burden of Disease study ${ }^{29}$ ), and drug costs for therapy (based on per-unit global buyer cost estimates from the International Medical Products Price Guide ${ }^{192}$ ) were integrated over the simulated lifecourse of all people with diabetes who were alive or born during the next 10 years using the projected estimates for the population and those with diabetes, as per standard cost-effectiveness guidelines. ${ }^{230}$ Additional service delivery costs were assumed to be the same for each strategy. DALYs and costs were discounted at a $3 \%$ annual rate, the rate used by WHO, ${ }^{231}$ and costs are expressed in 2016 US dollars.
With the TTT strategy, the estimated total drug costs in the countries studied would be US\$1346.6 million (95\% CI \$471.1-2206.7 million; 2016 prices) over the simulated period of 10 years, whereas with the BTT strategy, the estimated total drug costs would be higher at $\$ 1407.9$ million (\$464.7-\$2332.5 million). In terms of total DALYs averted, the benefits of BTT were estimated to be superior (table 10), with BTT saving 1.89 million DALYs $(0 \cdot 89-3 \cdot 38$ million) and TTT saving $1 \cdot 16$ million DALYs $(0.55-2.07$ million) in the simulated period of 10 years in sub-Saharan African countries. With BTT, the mean costs per DALY averted were $\$ 743.4$ $(\$ 137 \cdot 5-\$ 2627 \cdot 2)$ for the BTT strategy and $\$ 1159 \cdot 5$ $(\$ 227 \cdot 6-\$ 4047 \cdot 5)$ for the TTT strategy (table 10).
These results show that there are potentially substantial benefits for scaling up diabetes services to address unmet need. The question remains as to whether countries will be able to strengthen health systems to respond to those needs.

\section{Service delivery models for managing diabetes in sub-Saharan Africa}

Encouraging examples of successful models of diabetes care are emerging in LICs and in sub-Saharan Africa. In this section, we explore these models and provide countrycase examples of innovative approaches introduced in sub-Saharan Africa to effectively manage diabetes to show what might be possible in the future. 


\begin{tabular}{|c|c|c|c|c|}
\hline & \multicolumn{2}{|c|}{$\begin{array}{l}\text { Malawi ( } n=8632000 \text { people aged } 30-70 \text { years, } \\
\text { about } 604000 \text { with diabetes) }\end{array}$} & \multicolumn{2}{|c|}{$\begin{array}{l}\text { Sub-Saharan Africa ( } n=318850000 \text { people aged } \\
30-70 \text { years, about } 24870000 \text { with diabetes) }\end{array}$} \\
\hline & $\pi T$ & $\mathrm{BTT}$ & TाT & $\mathrm{BTT}$ \\
\hline $\begin{array}{l}\text { Adults with diabetes recommended } \\
\text { treatment, thousands }\end{array}$ & $\begin{array}{l}522 \cdot 0 \\
(86 \cdot 4 \%[85 \cdot 4-87 \cdot 4 \%])\end{array}$ & $\begin{array}{l}534 \cdot 1 \\
(88 \cdot 4 \%[87 \cdot 4-90 \cdot 0 \%])\end{array}$ & $\begin{array}{c}23942 \cdot 7 \\
(96 \cdot 3 \%[95 \cdot 2-97 \cdot 3 \%])\end{array}$ & $\begin{array}{c}21688 \cdot 3 \\
(87 \cdot 2 \%[65 \cdot 6-100 \cdot 0 \%])\end{array}$ \\
\hline Blood pressure treatment & $\begin{array}{l}300 \cdot 9 \\
(49 \cdot 8 \%[40 \cdot 0-59 \cdot 6 \%])\end{array}$ & $\begin{array}{l}490 \cdot 6 \\
(81 \cdot 2 \%[72 \cdot 4-90 \cdot 0 \%])\end{array}$ & $\begin{array}{l}13800 \cdot 9 \\
(55 \cdot 5 \%[44 \cdot 6-66 \cdot 4 \%])\end{array}$ & $\begin{array}{c}19922 \cdot 6 \\
(80 \cdot 1 \%[54 \cdot 3-100 \cdot 0 \%])\end{array}$ \\
\hline Lipid treatment & $\begin{array}{l}478 \cdot 6 \\
(79 \cdot 2 \%[62 \cdot 6-95 \cdot 8 \%])\end{array}$ & $\begin{array}{l}490 \cdot 6 \\
(81 \cdot 2 \%[72 \cdot 4-90 \cdot 0 \%])\end{array}$ & $\begin{array}{c}20420 \cdot 1 \\
(82 \cdot 1 \%[67 \cdot 4-100 \%])\end{array}$ & $\begin{array}{l}19922 \cdot 6 \\
(80 \cdot 1 \%[54 \cdot 3-100 \cdot 0 \%])\end{array}$ \\
\hline Glycaemic treatment & $\begin{array}{l}300 \cdot 3 \\
(49 \cdot 7 \%[47 \cdot 9-51 \cdot 5 \%])\end{array}$ & $\begin{array}{l}313 \cdot 8 \\
(51 \cdot 9 \%[50 \cdot 0-53 \cdot 8 \%])\end{array}$ & $\begin{array}{c}12814 \cdot 1 \\
(51 \cdot 5 \%[51 \cdot 5-52 \cdot 7 \%])\end{array}$ & $\begin{array}{c}13387 \cdot 9 \\
(53 \cdot 8 \%[53 \cdot 8-55 \cdot 0 \%])\end{array}$ \\
\hline $\begin{array}{l}\text { Number of medications per person for } \\
\text { recommended treatment }\end{array}$ & $3 \cdot 5(3 \cdot 2-3 \cdot 8)$ & $4 \cdot 5(4 \cdot 3-4 \cdot 7)$ & $3.6(3.5-3.9)$ & $4 \cdot 3(4 \cdot 2-4 \cdot 5)$ \\
\hline $\begin{array}{l}\text { Percentage of those on glycaemic } \\
\text { treatment requiring insulin }\end{array}$ & $19 \cdot 4 \%(17 \cdot 4-21 \cdot 4 \%)$ & $20 \cdot 3 \%(18 \cdot 2-22 \cdot 4 \%)$ & $20 \cdot 1 \%(18 \cdot 7-21 \cdot 9 \%)$ & $21 \%(19 \cdot 6-22 \cdot 9 \%)$ \\
\hline \multicolumn{5}{|c|}{ Macrovascular events prevented per 10 years, thousands } \\
\hline Myocardial infarction & $2 \cdot 4(2 \cdot 2-2 \cdot 7)$ & $9 \cdot 6(7 \cdot 6-11 \cdot 4)$ & $108 \cdot 9(103 \cdot 8-122 \cdot 4)$ & $226 \cdot 2(182 \cdot 5-268 \cdot 3)$ \\
\hline Stroke & $3 \cdot 8(3 \cdot 5-4 \cdot 2)$ & $13 \cdot 7(11 \cdot 5-15 \cdot 8)$ & $174 \cdot 4(163 \cdot 0-189 \cdot 1)$ & $322 \cdot 8(276 \cdot 0-371 \cdot 3)$ \\
\hline \multicolumn{5}{|c|}{ Microvascular events prevented per 10 years, thousands } \\
\hline Blindness & $0.6(0 \cdot 2-2 \cdot 4)$ & $0 \cdot 6(0 \cdot 1-2 \cdot 3)$ & $26 \cdot 6(8 \cdot 7-105 \cdot 4)$ & $25 \cdot 9(5 \cdot 5-95 \cdot 9)$ \\
\hline End-stage renal disease & $0.6(0.1-0.5)$ & $0.6(0 \cdot 0-0 \cdot 3)$ & $26 \cdot 6(3 \cdot 6-23 \cdot 9)$ & $25 \cdot 9(0 \cdot 5-11 \cdot 4)$ \\
\hline Amputation & $1 \cdot 5(0 \cdot 2-2 \cdot 7)$ & $1 \cdot 6(0 \cdot 3-4 \cdot 2)$ & $66 \cdot 4(10 \cdot 7-118 \cdot 8)$ & $69 \cdot 4(11 \cdot 5-182 \cdot 8)$ \\
\hline Deaths averted per 10 years, thousands & $4 \cdot 7(0 \cdot 5-10 \cdot 2)$ & $14 \cdot 0(1 \cdot 6-31 \cdot 7)$ & $212 \cdot 6(25 \cdot 7-454 \cdot 2)$ & $358 \cdot 0(40 \cdot 4-833 \cdot 7)$ \\
\hline $\begin{array}{l}\text { Number needed to treat to prevent } \\
\text { one macrovascular event per } 10 \text { years }\end{array}$ & $83 \cdot 9(75 \cdot 3-92 \cdot 4)$ & $23 \cdot 0(19 \cdot 6-27 \cdot 9)$ & $84 \cdot 5(76 \cdot 9-89 \cdot 7)$ & $39 \cdot 5(33 \cdot 9-47 \cdot 3)$ \\
\hline $\begin{array}{l}\text { Number needed to treat to prevent } \\
\text { one microvascular event per } 10 \text { years }\end{array}$ & $193 \cdot 2(91 \cdot 8-1042 \cdot 9)$ & $189 \cdot 2(78 \cdot 6-1355 \cdot 5)$ & $200 \cdot 3(96 \cdot 5-1041 \cdot 9)$ & $178 \cdot 9(74 \cdot 8-1238 \cdot 0)$ \\
\hline \multicolumn{5}{|l|}{ Cost and cost-effectiveness } \\
\hline $\begin{array}{l}\text { Total drug costs per } 10 \text { years, } \\
\text { thousand US\$ ( } 2016 \text { values) }\end{array}$ & $\begin{array}{l}31443 \cdot 3 \\
(10612 \cdot 4-51794 \cdot 0)\end{array}$ & $\begin{array}{l}33577 \cdot 7 \\
(12020 \cdot 7-53600 \cdot 4)\end{array}$ & $\begin{array}{l}1346617 \cdot 9 \\
(471138 \cdot 5-2206655 \cdot 0)\end{array}$ & $\begin{array}{l}1407869 \cdot 7 \\
(464664 \cdot 0-2332519 \cdot 3)\end{array}$ \\
\hline $\begin{array}{l}\text { Total DALYs averted per } 10 \text { years, } \\
\text { thousands }\end{array}$ & $25 \cdot 8(11 \cdot 6-46 \cdot 7)$ & $73 \cdot 2(36 \cdot 5-124 \cdot 6)$ & $1161 \cdot 3(545 \cdot 2-2070 \cdot 2)$ & $1893 \cdot 9(887 \cdot 8-3380 \cdot 4)$ \\
\hline \$ per DALYs averted & $1221(227 \cdot 3-4476 \cdot 8)$ & $459(96 \cdot 5-1466 \cdot 9)$ & $1159 \cdot 5(227 \cdot 6-4047 \cdot 5)$ & $743 \cdot 4(137 \cdot 5-2627 \cdot 2)$ \\
\hline \multicolumn{5}{|c|}{$\begin{array}{l}\text { Data are } \mathrm{n}(\%[95 \% \mathrm{CI}]) \text { or } \mathrm{n}(95 \% \mathrm{Cl}) .95 \% \text { Cls were estimated from } 10000 \text { repeated simulations of the model in which the probability distributions of all input parameter } \\
\text { values were repeatedly sampled to identify uncertainty in the outcome metrics shown. Data to represent sub-Saharan African countries were drawn from cohorts from Ghana } \\
\text { and South Africa obtained from the WHO Study on Global Ageing and Adult Health. }{ }^{232} \text { For more detail on methods, see panel } 7 \text { and appendix } 1 \text {. BTT=benefit-tailored therapy. } \\
T T T=\text { treat-to-target strategy. DALY=disability-adjusted life-year. }\end{array}$} \\
\hline
\end{tabular}

We reviewed published studies of existing approaches to diabetes care in sub-Saharan Africa (see appendix 1 for methods). We also did case studies to examine current practices in seven countries (Botswana, Ethiopia, Kenya, Malawi, Mali, Rwanda, and South Africa; see appendices 2-8 for details), using a framework that has been used previously to analyse different disease programmes ${ }^{233-237}$ in several settings ${ }^{238}$ and that was adapted for diabetes. Additionally, we examined how existing and new technologies could be harnessed to help improve management of diabetes in sub-Saharan Africa.

Our literature review identified 467 studies, of which 32 from 11 different countries (Cameroon, Eritrea, Ethiopia, Ghana, Kenya, Malawi, Mozambique, South Africa, Sudan, Tanzania, and Uganda) met the inclusion criteria (ie, if they described or evaluated a new or previously existing model of diabetes care in sub-
Saharan Africa; appendix 1). The review showed that, in many sub-Saharan African countries, diabetes care is still mostly available only in the hospital setting, requiring patients to travel great distances to access services and follow-up care important for effective diabetes management. However, several countries have seen success with the introduction of new, decentralised care-delivery models for management of diabetes (eg, in primary health centre, community, or home settings), with an emphasis on use of nurses and community health workers, patient education, and community engagement.

Cameroon and Tanzania have introduced communitybased management of diabetes. ${ }^{239}$ In Cameroon, facilitybased interventions for high blood pressure and diabetes, involving task shifting and nurse-led care in rural health districts, significantly improved patient retention in cardiovascular disease management programmes. ${ }^{240}$ 
Similarly, in Kenya, management of high blood pressure and diabetes has been delegated to rural primary healthcare clinics, with good retention rates and control. ${ }^{241}$ In Ethiopia, physicians and diabetes-trained nurses travel from hospitals to rural medical centres for training and care provision, with early encouraging results in improving access to services. ${ }^{242,243}$

In the public sector in South Africa, a chronic disease outreach programme, which used nurses in primary health centres to provide educational and follow-up advice to patients with diabetes, improved early detection and referral of high-risk, poorly controlled patients to specialist centres..$^{24}$ Decentralisation of diabetes management to the community level was also successful in the private sector in South Africa, where the care of diabetes was transferred to physicians working in community-based primary health centres affiliated with a diabetes and endocrinology centre. The scheme used community-based capitation and a financial risk-sharing model for diabetes and led to major reductions in hospital admissions for both acute metabolic emergencies and all causes, as well as reduced costs, delayed progression of microvascular complications, and improved outcomes. ${ }^{245}$

Nurse-led diabetes care, with a nurse-led protocol and education-based system, was also shown to be successful in rural KwaZulu-Natal in South Africa, with improved control of $\mathrm{HbA}_{1 \mathrm{c}}$ and increased satisfaction for patients, their families, and health workers. ${ }^{246,247}$ Although the improvements in glycaemic control achieved at 18 months following the introduction of that scheme were not sustained at 48 months, mean $\mathrm{HbA}_{1 \mathrm{c}}$ concentrations at 48 months were lower than at baseline. ${ }^{247}$ Similar to South Africa, the introduction of protocol-driven, nurse-led management of diabetes in primary health-care centres in rural and urban Cameroon also led to improvements in glycaemic control and blood pressure. ${ }^{248}$ However, a study ${ }^{249}$ of a community-health-worker model of outpatient care introduced in South Africa for diabetes and hypertension-which involved monthly home visits, counselling services, and access to monthly supplies of medication-showed improved control of hypertension but not of diabetes, with 11 (26\%) of 42 patients at the clinic showing improved glycaemic control compared with only two (9\%) of 22 patients visited at home by community health workers.

Combined with decentralisation of services, plans to specifically integrate care have been successful. In Cameroon, integration of care for high blood pressure and diabetes was effectively achieved in eight rural health districts by task shifting to health-care facilities with nonphysician clinicians, with improved control of risk factors in patients attending those services. ${ }^{249}$

In South Africa, home glucose monitoring with urine testing was introduced 30 years ago, including for illiterate patients with diabetes, with good compliance and lower random glucose levels in compliers than in non-compliers. ${ }^{250}$ In Kenya, a home glucose monitoring programme that used mobile phones to enable community health workers to regularly communicate with patients to modify the dose of insulin injections helped to improve $\mathrm{HbA}_{1 \mathrm{c}}$ concentrations. ${ }^{251,252}$

Patient education has also been used in several settings to improve services across the care continuum and to decentralise care away from hospitals to the patient level. For example, in Cameroon, motivational counselling and education were integrated into a screening programme to improve rates of follow-up for patients newly diagnosed with diabetes. ${ }^{253}$ In South Africa, group education was used at community health centres to improve patients' knowledge and management of diabetes, although no improvements were seen in diabetes self-care activities, weight loss, $\mathrm{HbA}_{1 c}$ concentrations, quality of life, selfefficacy, locus of control, blood pressure, waist circumference, or total cholesterol levels. ${ }^{144}$ Another study ${ }^{254}$ from South Africa showed that a group education programme for patients with type 2 diabetes could be implemented in rural areas with a dietitian or health promoter to provide a supportive environment for patients to learn and cope. That programme led to significant improvements in adherence to a diabetesappropriate diet, physical activity, foot care, and the perceived ability to educate others, although no significant change was seen in smoking or adherence to medication. ${ }^{254}$ In Tanzania, a hospital-based education programme for children with type 1 diabetes about symptom management, correct insulin storage, and insulin administration led to reductions in severe hypoglycaemia, but no improvement in $\mathrm{HbA}_{1 c}$ concentrations. ${ }^{255}$ In Mozambique, twinning programmes have been used to successfully establish patient education programmes and to improve their effectiveness. ${ }^{185}$

In addition to patient education, health-provider education at hospitals and primary health-care centres, and at the community level, has been used to improve early recognition of diabetes and diabetic sequelae and to enhance disease management in sub-Saharan Africa. In Tanzania, where $70 \%$ of leg amputations occur in patients with diabetes, ${ }^{109}$ training of health-care personnel at different levels in diabetic foot management has led to improved case finding, earlier referrals, establishment of well functioning foot clinics, and strengthened management of diabetic foot ulcers, with better health outcomes. ${ }^{109}$ In Eritrea, a cooperative diabetes project that emphasised multidisciplinary training of physicians, laboratory scientists, diabetes nurse practitioners, patient educators, and dietitians, and improved quality of laboratory services, led to improved management of diabetes, with better $\mathrm{HbA}_{\mathrm{lc}}$ concentrations. ${ }^{256}$ Multidisciplinary training for diabetes, involving physicians, dietitians, nurse educators, and pharmacists, has also been introduced in Ghana to strengthen diabetes services, ${ }^{257}$ while training of patient educators has been introduced in Sudan..$^{258}$ 
In sub-Saharan Africa, electronic medical records, ${ }^{259}$ treatment protocols, and guidelines ${ }^{260}$ have facilitated the development of primary care, community, and homebased service models. For example, Malawi has successfully used existing service-delivery platforms and expertise in tuberculosis management, which has well established treatment guidelines and monitoring and reporting mechanisms, to introduce chronic disease management and reporting for diabetes. ${ }^{261}$

Integrating care of diabetes and hypertension into existing chronic care models for HIV has also been deemed a promising strategy. South Africa has used-in urban and peri-urban townships of the metropolitan region of Cape Town in Western Cape-mobile units for HIV counselling and testing as an entry point for combined screening of high blood pressure and diabetes, with a high yield of new cases, although linkage to care and follow-up was challenging. ${ }^{225}$ Community-based HIV-testing campaigns were used in Uganda to simultaneously offer diagnostic, preventive, treatment, and referral services for HIV, malaria, tuberculosis, high blood pressure, and diabetes, with effective linkage to care. ${ }^{262}$ In a study done in Kenya, ${ }^{252} \mathrm{HIV}$ counsellors were trained to screen for diabetes and high blood pressure in home-based screening, and district hospital-based staff to do community-based screening; both approaches showed effective uptake, although, in both, follow-up levels were low, with only one-fifth of all patients screened returning after a random glucose test.

Diabetes management in Africa is strongly reliant on the non-physician health workforce, including nurses, community health workers, and health extension workers, as well as traditional healers in some settings. Several countries, such as Botswana, Ghana, Ethiopia, Kenya, Malawi, Mali, Rwanda, Tanzania, and South Africa, have introduced innovative approaches to address resource constraints when managing diabetes (see panel 8 for a summary of initiatives for and barriers to providing diabetes care in selected countries in subSaharan Africa). In-country case studies for Botswana, Ethiopia, Kenya, Malawi, Mali, Rwanda, and South Africa See Online for appendices 2-8 are published as appendices 2-8.

\section{Harnessing new technologies to improve}

diabetes care in sub-Saharan Africa

Improving access to existing technologies for better diabetes care in sub-Saharan Africa

New technologies for diabetes care have been developed for use in resource-rich health systems, but not for those in sub-Saharan Africa. In the region, the necessary technologies either do not exist (requiring the development of low-cost health technologies), or technologies exist but are not accessible because of high costs, or accessible technologies are not adopted because of health system barriers.

Most health technologies used to diagnose, monitor, and treat diabetes and its complications are not affordable for patients in sub-Saharan Africa. Patients experience difficulties in accessing health centres and, when they do, incurimpoverishing expenditures. ${ }^{17,617,263-266}$ Increased availability of low-cost and accurate diagnostics for point-of-care testing of blood glucose, $\mathrm{HbA}_{1 c}$, glycosuria, and proteinuria could improve screening, diagnosis, treatment, and monitoring of diabetes and help to mitigate access constraints in delivering effective diabetes care, particularly when combined with a distributive model of primary-healthcentre-based and community-based services provided by community health workers (panel 8).

Ensuring timely and affordable access to existing costeffective technologies, such as appropriate forms of insulin ${ }^{267}$ medicines, miniaturised blood glucose sensors, and strips for testing of urinary glucose or protein, is a challenge in sub-Saharan Africa. Improved technology assessment, procurement, and supply-chain management can help to achieve greater value for money and timely delivery of available medicines to avoid treatment interruptions and expand access. ${ }^{268}$

Strategies for improved use of cost-effective technology should emphasise both health technologies (eg, new therapeutics, diagnostics, and medical devices) and technologies for health (eg, communication and transport, which affect health). These strategies should foster investments in hard (infrastructure and equipment) and soft (information and communication technology [ICT], with data analytics as an example) technologies.

In sub-Saharan Africa, ICT has been used variously in HIV and tuberculosis programmes, ${ }^{269}$ but, overall, its use in health systems is low. Diabetes registries, which form the mainstay of diabetes care platforms in HICs, are only available in six (17\%) of 35 countries that responded to the WHO survey in the entire WHO African region..$^{8}$ One possibility is to extend existing databases for HIV and tuberculosis to include diabetes and NCDs. In countries where no such systems exist, development of future-proof diabetes registries should be prioritised. The absence of legacy systems provides an opportunity to introduce cost-effective ICT solutions that use mobile telephones to facilitate data sharing. ${ }^{270-272}$ For example, existing solutions using cloud technologies offer an important opportunity to capture and integrate real-time data across multiple mobile phones, point-of-care devices, laboratory diagnostics, and electronic health records (panel 9).

\section{Improving adoption and dissemination of new health technologies for diabetes care in sub-Saharan Africa} Factors that hinder adoption and dissemination of new technologies in sub-Saharan Africa include imprecise definition of the problem being addressed; complexity and scalability of the technology or intervention; resistance from the adoption system (eg, health professionals and service users); characteristics of the health system that create rigidities or provide inadequate 
Panel 8: Health services for diabetes in sub-Saharan Africa: a summary of the Commission's case reports of seven countries (see appendices 2-8 for more detail and references)

In Botswana, patients with diabetes have access to general nursing, psychological, and social work services, both in the private and public sectors. Community health workers and diabetes youth leaders provide education and public health screening campaigns.

In Ethiopia, diabetes services are delivered mainly at primary health centres. Specialist clinics located in the major university teaching hospitals also provide diabetes care by endocrinologists or general internists. Nurses, health officers, and general practitioners provide most of the diabetes care in health centres and general hospitals. In 2014, an estimated six of ten health facilities, excluding health posts, provided diabetes care, although most patients with diabetes are undiagnosed.

After the launch of a national diabetes strategy in 2010, Kenya began to expand diabetes services, with training of health-care professionals and community health workers, development of national guidelines, and provision of diabetes screening. However, services at the level of the community and primary health centre are hampered by the inconsistent availability of drugs and diagnostic equipment.

Malawi has low numbers of health personnel and resource shortages, and patients with diabetes are underdiagnosed and poorly controlled. However, Malawi is piloting the WHO Package of Essential Noncommunicable Disease Interventions for Primary Health Care in Low-Resource Settings and set a target to give 1000 health workers specialist training by 2016. Malawi has introduced new service-delivery models for diabetes that mimic the directly observed treatment (short-course) approach used in tuberculosis care and home-based care and peer support used in management of HIV.

Rwanda is introducing clinical non-communicable disease (NCD) services and care packages across all health facilities and an NCD clinic model in district hospitals and health centres. The number of people living with diabetes and requiring close follow-up has increased. To address the shortage of health professionals and increasing demand for health care, Rwanda has introduced pre-service and in-service training for existing staff in the management of NCDs, started a programme of task shifting to transition chronic disease management to the home setting, and created a new cadre of health professionals (home-based care practitioners) at the community level to provide home-based services for diabetes and other NCDs.

In 2011, Tanzania established a National Diabetes Program as a collaboration between the Ministry of Health and Social Welfare and the Tanzania Diabetes Association-the implementing agency for the programme-that has used existing government facilities and staff to establish 148 diabetes clinics in zonal, regional, and district hospitals. Using funding from international agencies and the private sector, Tanzania's National Diabetes Program provides funding for equipment and training of personnel to provide care for around 800000 people with diabetes. Diabetes care is provided free of charge to patients, and the government mandates that diabetes drugs should be given free of charge to patients using district and regional diabetes clinics.

In South Africa, diabetes care is provided in the public and private sectors, but huge inequities exist: the public sector provides for $84 \%$ of the population and spends US $\$ 140$ per person each year (4.2\% of gross domestic product [GDP]), whereas the private sector provides for $16 \%$ of the population and spends $\$ 1400$ per person each year ( $4.3 \%$ of GDP). A network of national health laboratories provides services to more than $80 \%$ of the population for all standard diabetes-related investigations, but not all primary health clinics have access to standard diagnostic equipment because of budgetary constraints. incentives; and poor recognition of the challenge of diabetes in the broader context (among the population and politicians), such that it is not considered an urgent and major societal challenge. ${ }^{281,282}$

As we have discussed, emerging care-delivery models for diabetes in sub-Saharan Africa have distinctive features: a public health approach, with simplification and decentralisation of care to primary health centres and communities and with strong reliance on a nonphysician health workforce (including nurses, community health workers, and health extension workers); community involvement; peer support; and self-management. Expanded access to existing technologies can help sub-Saharan Africa not to replicate western models of care, which often favour an expert-led approach, but to foster innovative delivery models that reflect the African context and to build on experience of the innovations in care delivery with HIV/AIDS. ${ }^{283,284}$

\section{Creating a successful response to diabetes in sub-Saharan Africa \\ Aligning with global targets}

Health systems in sub-Saharan Africa are unprepared for the rapidly increasing burden of diabetes. Consequently, many people go undiagnosed, and those who are diagnosed are not screened for comorbidities, do not receive treatment, or are not adequately controlled. The resultant morbidity and mortality lead to immense human, economic, and societal losses.

The increasing prevalence of diabetes in sub-Saharan Africa reflects not just greater numbers of people with the disease, but also a changing phenotype. The approach to managing such a complex and heterogeneous disorder has to be multisectoral, with education, engagement, and cooperation of individuals, and with awareness and education of the community. Specific therapeutic measures are not enough; successful management of 
For more on SDG 3 see http://www.un.org/ sustainabledevelopment/health

For more on the Portable Eye Examination Kit see http://www.peekvision.org

For more on epiCam see http://www.epipole.com/epicam

For more on SUDOSCAN see http://www.sudoscan.com For moreon NC-stat DPNCheck see http://www.dpncheck.com diabetes in sub-Saharan Africa requires an enabling medical, social, and political context within which effective prevention, screening, diagnosis, treatment, and lifelong care can be delivered. In this respect, lessons can be learned from the HIV response in Africa, which successfully brought together governments, civil society, health-care providers, communities, donors, and the private sector.

The burden of diabetes in sub-Saharan Africa and its impact on individuals, populations, health systems, and economies means that policy makers must act to bring together wide-ranging stakeholders to spur action at country and global levels around a set of ambitious yet achievable targets, linked to those set in SDG 3: "ensure healthy lives and promote well-being for all at all ages". Of the 13 targets set for SDG 3, six are most readily applicable to diabetes in sub-Saharan Africa: (1) by 2030, reduce by one-third premature mortality from NCDs through prevention and treatment; (2) achieve universal health coverage, including financial risk protection, access to quality essential health-care services and access to safe, effective, quality, and affordable essential medicines and vaccines for all; (3) strengthen the implementation of the WHO Framework Convention on Tobacco Control in all countries, as appropriate; (4) support the research and development of vaccines and medicines for the communicable diseases and NCDs that primarily affect

Panel 9: Possibilities for affordable, cost-effective technologies for diabetes care in sub-Saharan Africa

Technologies to support diabetes diagnosis and management

Low-cost diagnostic devices that enable point-of-care testing of blood glucose, $\mathrm{HbA}_{1 c}$ glycosuria, and proteinuria could be used for screening, diagnosis, treatment initiation, and monitoring. Yet, although low-cost, affordable devices do exist, such devices are produced by numerous manufacturers and often little compatibility exists between the necessary elements of the equipment (eg, blood glucose sticks) needed to use such devices, even for some manufacturers' own models. Encouraging diagnostic device manufacturers to make changes towards greater interoperability will necessitate a more centralised approach to procurement of diagnostic devices used in the management of non-communicable diseases (NCDs). Moreover, international bodies such as WHO have an important part to play in developing the technical standards required for improved integrated diagnostic infrastructures for NCDs such as diabetes, just as these bodies did in strengthening diagnostic service infrastructures for infectious diseases such as HIV and tuberculosis. Improved interoperability of developed technologies that have interchangeable disposable components is needed to ensure that low-cost models can be used in low-income and middleincome countries.

Results from such devices could be used in combination with mobile phones or smartphones to target messaging for preventive interventions, ${ }^{273}$ to communicate test results to patients, ${ }^{274}$ or to self-monitor diabetes (to help to improve adherence) ${ }^{275}$ Affordable diagnostic and communication technologies could help to transform diabetes care, as they have done for HIV management in resource-poor settings..$^{27,277}$

Technologies to support screening for diabetes complications

Effective early detection of diabetic retinopathy, which might reduce the risk of blindness by $95 \%$, currently requires both clinical staff with ophthalmological training and costly equipment to carry out eye examinations. Both requirements are a major obstacle to care, as shown by the case of Malawi, which has 17.8 million inhabitants but just nine ophthalmologists. ${ }^{278}$ New devices, such as the Portable Eye Examination Kit, which uses a smartphone, and the hand-held epiCam device, which captures images digitally for store-and-forward using a laptop computer, offer the possibility of screening for diabetic retinopathy by health workers in primary health centres, community-based services, and remote rural areas. Although these devices rely on expert analysis for interpretation of fundus images, diagnostic algorithms could be used to remotely analyse and grade images at low cost. For example, automated grading of fundus photographs is done within established services, including the Scottish National Diabetic Retinopathy Screening Programme, and has been studied in Nakuru, Kenya. ${ }^{279}$ However, reliable supporting infrastructure needs to be in place to allow the widescale adoption of such technology.

Peripheral neuropathy is another important complication of diabetes that has an urgent need for new diagnostic tools for early detection; the prognosis of diabetic peripheral neuropathy is poor if not diagnosed early. However, accurate diagnosis of diabetic peripheral neuropathy represents a major challenge, even in the context of resource-rich health systems. ${ }^{280}$ The development and adoption of new non-invasive diagnostic devices that enable point-of-care testing and do not require specialised training to use - eg, SUDOSCAN and NC-stat DPNCheck-could improve screening and early detection of diabetic peripheral neuropathy in resource-poor settings.

\section{Technologies to support diabetes surveillance}

Cloud-based systems offer the possibility of capturing data from multiple sources and devices in real-time and integrating them. Data cloud solutions are low cost and more scalable than traditional data storage systems (which are reliant on hardware requiring constant electricity supply and regular servicing). By integrating data from multiple devices and sources, cloud solutions can help to manage the complex data needed for management of diabetes across multiple facilities and over long periods of time, and help to improve understanding of the epidemiology of diabetes and responses to interventions. 
developing countries, and provide access to affordable essential medicines and vaccines; (5) substantially increase health financing and the recruitment, development, training, and retention of the health workforce in LICs, especially in the least developed countries; and (6) strengthen the capacity of all countries, in particular developing countries, for early warning, risk reduction, and management of national and global health risks (not just for infectious diseases, but also for diabetes and other NCDs).

Additionally, countries in sub-Saharan Africa should embrace the nine voluntary targets adopted at the 65th World Health Assembly by member states as part of the WHO Global Action Plan for the Prevention and Control of NCDs 2013-20: to reduce premature mortality due to NCDs by $25 \%$, to reduce physical inactivity by $10 \%$, to reduce tobacco consumption by $30 \%$, to achieve a $25 \%$ reduction in high blood pressure, to achieve $80 \%$ coverage of essential medicines and technologies for NCDs, and to achieve $0 \%$ increase in the prevalence of obesity and diabetes by the year 2025. ${ }^{187}$ A Global Monitoring Framework underpins the Global Action Plan, with 25 indicators that are monitored by member states, of which 12 are relevant to diabetes and can be used to monitor achievements in sub-Saharan Africa. ${ }^{285}$

Achieving the targets set in SDG 3 and the Global Action Plan would undoubtedly help to transform the fight against diabetes in sub-Saharan Africa; avert needless suffering and death; and reduce the economic burden of diabetes on individuals, households, and societies. The crucial components of an effective response are solidarity and collective action at local, national, African, and global levels, with clear responsibilities for stakeholders as part of a collective response. The chief elements of this response, on which the Commissioners are in full agreement, are laid out below. We have also described operational targets that are achievable by 2020, 2025, and 2030 in panel 1.

\section{Role of governments}

Ultimately, governments should respond to the needs of their populations. In addition to enabling access to health services, governments should consider introducing public health measures to reduce NCDs, including banning smoking in public places, restricting advertising of unhealthy foods and beverages, creating or increasing taxes on cigarettes and sugar-sweetened beverages, and limiting portion sizes of sugar-sweetened beverages. ${ }^{43,286}$

A health-literate population is crucial for generating demand for health interventions, improving access to health systems, and ensuring uptake of preventive measures. Much health literacy in sub-Saharan Africa has come from successful media campaigns via radio and poster adverts and education through interaction with community health workers. These campaigns have generally focused on prevention of HIV (eg, condom use) and malaria (eg, use of insecticide-treated bednets), ${ }^{287}$ and have led to increased use of preventive interventions. Investment and research is now needed to build on these initiatives and to increase population awareness of diabetes and generate health-care demand for diabetes and NCDs. Therefore, we recommend that countries in sub-Saharan Africa research and develop locally appropriate media campaigns to educate the public about the symptoms of type 1 and type 2 diabetes and encourage those with symptoms to seek care. In particular, media campaigns should be deployed to educate citizens about preventive lifestyle measures, combined with government regulations that restrict advertisements for unhealthy foods.

We also recommend that governments should allocate sustainable funding to tackle the diabetes epidemic. As noted earlier, we have estimated that, in 2015, the economic burden of diabetes in sub-Saharan Africa was US $\$ 19.45$ billion, or $1.2 \%$ of cumulative GDP of the whole sub-Saharan African region. Unchecked, this economic burden is projected to increase to between $\$ 35.33$ billion and $\$ 59.32$ billion by 2030 (figure 10 ).

Given the strong causal link between NCDs (including type 2 diabetes), sugar-sweetened beverages, salt, and tobacco, we strongly advise countries to consider raising revenues by taxing these products to fund health systems. Although all too often considered a disease of the rich, the burden of type 2 diabetes in sub-Saharan Africa is increasingly borne by the poor. ${ }^{21}$ Making available and encouraging healthy choices, and taxing unhealthy choices, should help to promote the health of the poorer sectors of society, while increasing revenue for treasuries to finance health systems and expand access to effective health care.

Such taxes should be combined with assessments of national health systems and actions to establish effective and efficient responses to the burden of diabetes to further expand fiscal space. Assessments should detail the burden of diabetes and its comorbidities, and their management at all levels of the health system, and involve analyses of the capability of human resources, the availability and costs (to individuals and health systems) of drugs and equipment, the functioning of supply-chain management, and how the services and platforms developed for communicable diseases can best be leveraged to also provide services for diabetes. In line with this recommendation, governments should prioritise data collection for the improvement of population health. Integrated digital health information systems are urgently needed to capture data about diabetes, its comorbidities, and their management in health systems, with timely analysis to inform planning and improve care.

Diabetes can be treated cost-effectively and its sequelae prevented if the disease is promptly diagnosed. The medicines required are on the WHO 2015 essential medicines list and are off-patent and affordable. 
Additionally, angiotensin-converting enzyme inhibitors for protection against microvascular disease should be made widely available (indeed, enalapril is currently included in the WHO list of essential medicines, albeit as an antihypertensive). ${ }^{288}$ Unlike other essential medicines used for diabetes management, generic forms of insulin are generally not widely available. However, countries can purchase vials of human insulin at prices similar in cost per person each year to that paid for a 1 year supply of fixed-combination antiretroviral treatment. Given the absolute need for insulin treatment for type 1 diabetes, and the increasing need for insulin to treat type 2 diabetes, human insulin should be widely available for all those who need it. We believe that, in the context of LICs in sub-Saharan Africa, more expensive analogue insulins do not provide enough extra benefit to justify their current costs. ${ }^{149}$ Considering treatment, we urge countries to make available all necessary medicines for diabetes, hypertension, and cardiovascular disease on the WHO essential medicine list at no cost to all patients who need them. Although newer treatments for type 2 diabetes might have some benefit over standard oral antihyperglycaemic agents and insulin, these treatments could be considered a worthwhile investment for the public sector only once the countries have been able to provide essential services and medicines for diabetes (table 1).

Human resource shortage is a crucial challenge, and there is an urgent need to educate and train all groups of health professionals-ranging from community health workers to specialist physicians-in the management of diabetes at all stages of the care continuum to enable increased detection and improved treatment. The need for training is especially great in areas where there is already substantial contact with the health system-for example, in children so that diagnosis of type 1 diabetes is not missed, with fatal consequences; in areas of maternal health, to ensure diagnosis and treatment of gestational diabetes (panel 4); and in communicable diseases, to ensure effective management of interactions between diabetes and communicable diseases. Once recognised, diabetes-especially type 2 diabetes-can be effectively managed. It is generally more cost-effective to train existing staff to diagnose and treat diabetes than to invest in highly trained specialists.

The sequelae of diabetes are insidious and present late in the disease course. Their effective management requires yearly monitoring at well equipped health centres with health-care professionals able to manage glycaemia and other cardiovascular risk factors and screen for complications to improve macrovascular and microvascular outcomes. It is essential that adequate referral pathways are in place to ensure regular monitoring and management of complications.

To minimise out-of-pocket costs from attending multiple clinics, services for diabetes, other cardiovascular risk factors, and chronic complications should be integrated wherever possible.

Overall, governments should work towards Target 3.8 included in the SDGs: "achieve universal health coverage, including financial risk protection, access to quality essential health-care services and access to safe, effective, quality and affordable essential medicines and vaccines for all".

\section{Role of civil society}

Civil society has a crucial role in catalysing change to improve access to health care and in holding governments to account. Advocacy from civil society was instrumental in the global movement for HIV, which prompted the convening of a special session of the UN General Assembly in 2001 and led to the first UN declaration focusing on a disease. ${ }^{289}$ Civil society could frame diabetes as an integral part of the global commitments to addressing NCDs and achieving universal health coverage, given links between diabetes and so many other risk factors and conditions, and use this narrative to build awareness and mobilise support among a broad range of stakeholders.

Effective deployment of civil society requires strengthening and expansion of networks and improved modes of communication. In many countries in subSaharan Africa, diabetes organisations have an important role in educating the public, health-care providers, and governments about diabetes (see in-country case studies in appendices 2-8). We recommend that diabetes organisations should continue to promote the improvement in management of diabetes across subSaharan Africa by tracking progress towards objectives set out in national NCD or diabetes plans, and by holding governments to account for their implementation. However, funding is needed to ensure sustained engagement of civil society. We urge donors and diabetes organisations in other world regions to consider funding or partnering with foundations in sub-Saharan Africa to enable mutual learning, strengthen the agenda for change, and ensure sustainability. Global NCD advocacy organisations-for example, the IDF, NCD Alliance, and World Heart Federation-have a key role in supporting local organisations in sub-Saharan Africa.

Although not known for certain, it is feared that many people with type 1 diabetes die undiagnosed or through lack of access to treatment. Civil society should be involved in raising awareness of type 1 diabetes and should ensure that government guarantee a supply of insulin to all patients, not just children.

\section{Role of international donors}

Although NCDs were identified as a priority in the 2011 UN High-Level Commission, ${ }^{290}$ with renewed commitment in SDG 3, they still do not feature prominently on the agendas of most global health funders. ${ }^{14}$ To date, few donor agencies have provided assistance for NCDs or for diabetes specifically. 
We do not know whether obesity and diabetes have received so little attention because they are seen as problems of lifestyle rather than of health, or because infectious disease is seen as a greater menace. Alternatively, NCDs might be less easy for international donors to market to their supporters as worthy areas for investment. Yet, evidence strongly suggests that overweight, obesity, and diabetes are affected more by environment than individual factors. ${ }^{291}$ Investment in management of NCDs is imperative if the health gains achieved in many countries in sub-Saharan Africa during the Millennium Development Goal period (2000-15) because of improvements in maternal, neonatal, and child health, and communicable diseases in that regionare to be sustained. Hence, donors should increasingly invest in evidence-based policies and health-systemswide strategies to address NCDs, communicable diseases, and maternal and child health.

\section{Role of global agencies}

As with international donors, global agencies have been slow to transition to a new world in which NCDs predominate. The 2011 UN High-Level Meeting on NCDs $^{15,290}$ produced the UN General Assembly Resolution ${ }^{292}$ committing UN member states to the prevention and control of NCDs. In 2013, at the 65th World Health Assembly, member states agreed to the aim of reducing premature mortality from NCDs by $25 \%$ by the year 2025 , relative to 2010 levels. ${ }^{293}$ However, in relation to action, apathy prevails; ${ }^{294}$ although a global coordination mechanism, a monitoring framework, an action plan, and a UN interagency task force have been established, their benefits to those living with NCDs is not sufficiently clear, given that targets are supposed to be met in 2025 (in only 8 years).

WHO needs to be engaged in the production of straightforward guidelines for countries looking to improve NCD prevention, diagnosis, and care. For example, lists of essential diagnostics for NCDs (akin to the WHO essential medicines list) could be very valuable.

WHO is well respected in sub-Saharan Africa and is well placed to have a leadership role. WHO also has the legitimacy to work with member states, other international agencies, and civil society organisations to mount an urgent and coordinated response to diabetes. However, this opportunity can only be realised if WHO acts on criticisms ${ }^{295}$ and lessons from the Ebola crisis to emerge as a leader in the battle against diabetes and NCDs. In addition to leading the response in countries, an under-resourced WHO should follow a collegial route, more widely involving external collaborators in efforts to support the diabetes response. ${ }^{24}$

The World Bank, with its development capability; the Global Fund, with its financing prowess; UNITAID, with expertise in innovative financing and creation of market dynamics to expand access to health technologies and medicines; Gavi, in effective partnerships to expand access to vaccines; the US Agency for International Development, in reproductive, maternal, and child health; and UNICEF, in building effective platforms for managing childrens' problems, could work together to catalyse an integrated response to NCDs.

To date, most non-governmental organisations (NGOs) in international health have focused on maternal, neonatal, and child health; on communicable diseases; and on providing health care in conflict settings. Although some NGOs, such as the IDF's Life for a Child programme and Santé Diabète, have focused on diabetes, these are exceptions. Encouragingly, Médecins Sans Frontières is increasing its involvement in NCDs. ${ }^{296}$

We encourage NGOs working in sub-Saharan Africa to recognise the importance of diabetes and obesity in the populations they care for and, when possible, work in concert with country health systems to find solutions to improve diabetes care. This recognition is particularly important for NGOs involved in communicable diseases and in improving maternal and neonatal health, wherein diabetes affects outcomes.

\section{Research, development, and innovation}

A global response to diabetes would not be possible without effective research and development and an innovation agenda to strengthen health systems, develop affordable technologies and medicines, and find innovative financing and service-delivery solutions. ${ }^{282,297}$ Throughout this report, we have highlighted the scarcity of evidence needed to inform an appropriate broadbased health system response to deal with diabetes, its complications, and other cardiovascular risk factors in sub-Saharan Africa. This deficit in knowledge is seen at all levels, from determining which measures to use to define diabetes, to defining the burden of disease, to making treatment decisions, and to planning costeffective health system development. Although some studies have been done in the region, those studies have often been small, out of date, or of poor quality. Our concern is that many of those studies have been assimilated into larger analyses and reports and thus will become instrumental in defining a response to diabetes in sub-Saharan Africa that might be wholly inappropriate to needs. There is a crucial need for highquality studies done in sub-Saharan Africa that are geared towards ensuring an effective health systems response. To date, funding of studies of diabetes and other NCDs in LMICs, including those in sub-Saharan Africa, has been woefully inadequate. However, given the urgency of the situation, we cannot advocate waiting to implement strategies for NCDs and diabetes until after the results of well done research studies have been reported. We do, however, strongly urge that all strategies are implemented in sub-Saharan Africa with implementation science methodology and are done on the background of a firm knowledge of baseline needs
For more on the Life for a Child programme see http://www.idf. org/lifeforachild

For Santé Diabète see http://www.santediabete.org/en For more on Médecins Sans Frontières see http://www.msf. org 
(including an accurate, population-level assessment of disease burden). We also believe that no programme of health care will truly maximise its potential unless there is local ownership - this necessitates co-development of research and implementation programmes with local users and providers. ${ }^{298}$

The shortage of health professionals is a major barrier to expansion of services in sub-Saharan Africa. Given resource constraints, rapid expansion in numbers of health professionals in the near future is not realistic. Hence, addressing human resource shortages will require a combination of strategies. The first of these strategies is task-shifting or task-sharing, which has been effectively implemented in sub-Saharan Africa to engage a broader group of health professionals in diabetes care (see earlier section on service delivery models for diabetes). The second strategy is to leverage novel technology advances in communications, including distance learning and e-learning, through use of online courses to improve the knowledge and competence of the existing health workforce. ${ }^{299}$ The third strategy, among others, is to use mobile technologies and SMS text messaging to improve management of communications and processes in health systems-for example, in communicating results, ${ }^{274}$ attending clinic appointments, ${ }^{276}$ and scaling up public health and prevention interventions. ${ }^{273}$ In addition to freeing up health-care-worker time, these measures also improve patient self-management of long-term illnesses. ${ }^{300}$

Innovative opportunities also exist for increasing financing resources. Financing initiatives such as the airline solidarity levy and Debt2Health, which have been used successfully for AIDS, tuberculosis, malaria, and children's immunisation programmes, offer possibilities for funding of health systems and diabetes care, ${ }^{301,302}$ as well as research. ${ }^{303}$

\section{Conclusion}

With rapid socioeconomic transitions occurring in subSaharan Africa, there is a risk that the increasing prevalence of diabetes (and associated NCDs) will overwhelm already struggling health services and have adverse consequences for individuals and economies. This Commission therefore chose to focus on this region as a priority, although our findings could be translated to other world regions facing similar challenges.

Our methods consisted of extensive reviews of the literature; soliciting of expert opinion; performance of primary research studies; and convening of Commissioners' meetings to discuss findings, challenges, and solutions. The major limitation that runs throughout all elements of the Commission is the scarcity of reliable data to inform findings. However, the findings presented here are robust to the current state of knowledge in the region, and this Commission should therefore serve as important reading for all members of the health-care community (from health-care workers, to ministers of health, and to heads of global development agencies) whose aim is to improve care for people with diabetes in sub-Saharan Africa. The finding of a paucity of reliable data is also a key message of the Commission, and more needs to be done to address the dearth of data in the region.

We conclude that sub-Saharan Africa is not prepared for the increasing burden of diabetes brought about by rapid and ongoing transitions. Effective management of diabetes in sub-Saharan Africa will require careful considerations about the expansion of services to meet current and future burden, while ensuring that services are integrated with those for other chronic diseases. The health, economic, and societal consequences of inaction will be huge. Decisive action is needed now, by all stakeholders, to address the scale and urgency of diabetes in sub-Saharan Africa.

\section{Contributors}

Commissioners-RA, JID, and, EAMG, as lead Commissioners, developed the original idea for the Commission, secured funding for the Commission meetings, chaired the Commission meetings, and wrote the Commission drafts. All Commissioners had input into the original Commission discussions that shaped the direction of the Commission, contributed ideas throughout the Commission process, and commented on Commission report drafts. New analyses for the Commission were contributed by TB (diabetes cascade analysis), DB (price and availability data of diabetes medications), and JSY (microsimulation model). NSL, MJN, and GDO were involved in writing the country-case reports for South Africa, Malawi, and Rwanda, respectively.

Analytics team members-Analytics team members contributed to new analyses for the Commission: SBa (the microsimulation model); JM-G and TB (the unmet need and care cascade analyses); TB, CB, EH, VS, and SV (the economics analysis); and IP (the SDI analyses).

Collaborative network-Contributors of specialty text and ideas to the Commission were ZGA (diabetic foot), PIB and MJB (diabetic eye disease), CNR (genetics of diabetes), and SJ (technological solutions). Contributors of the in-country case reports were MTA, SBe, AB, FPC, JD, AWE, CG, MNM, MAM, BMM, KJM, SPN, BN, PHP, SDP, CP, AR, SR, GS, ASi, ESS, KVA, and MW. Researchers for the health system section were BA, AAn, AAw, SBh, JC, PC, AC, JH, SSK, YK, RK, MAK, SCL, AL, CPM, MN, OLOO, OO, DS, ASh, and ASS.

\section{Declaration of interests}

ESS reports personal fees from GlaxoSmithKline, outside the submitted work. JID held the position of Editor of The Lancet Diabetes \& Endocrinology during some of the Commission's work. NSL reports funding for a diabetes educator from Roche Diagnostics and from Lilly laboratories, outside the submitted work. SDP reports personal fees from Abbott, outside the submitted work. All other authors declare no competing interests.

\section{Acknowledgments}

Funding for the Commissioners' meeting in Bellagio, Italy, was provided by the Rockefeller Foundation. The Harvard Medical School Center for Global Health Delivery provided funding for the Commissioners' meeting in Dubai. We thank Courtney Bridgeo, Sarah Linklater, Heather Van Epps, Gene Bukhman, and Joshua Salomon for their insights into the Commission's ideas and for their support throughout the process.

\section{References}

1 WHO. Global report on diabetes. Geneva: World Health Organization, 2016. http://apps.who.int/iris/bitstream/10665 /204871/1/9789241565257_eng.pdf (accessed June 12, 2017).

2 Geldsetzer P, Barnighausen T. Late-stage research for diabetes and related NCDs receives little funding: evidence from the NIH RePORTER tool. Lancet Diabetes Endocrinol 2017; 5: 91-92.

3 WHO. Diagnostic criteria and classification of hyperglycaemia first detected in pregnancy: a World Health Organization guideline. Diabetes Res Clin Pract 2014; 103: 341-63. 
4 World Bank. FAQs: global poverty line update. 2015. http://www. worldbank.org/en/topic/poverty/brief/global-poverty-line-faq (accessed March 10, 2017).

5 Riley L, Guthold R, Cowan M, et al. The World Health Organization STEPwise approach to noncommunicable disease risk-factor surveillance: methods, challenges, and opportunities. Am J Public Health 2016; 106: 74-78.

6 GBD 2015 Mortality and Causes of Death Collaborators. Global, regional, and national life expectancy, all-cause mortality, and cause-specific mortality for 249 causes of death, 1980-2015: a systematic analysis for the Global Burden of Disease Study 2015. Lancet 2016; 388: 1459-544.

7 World Bank. Health nutrition and population statistics. 2016. http://data.worldbank.org/data-catalog/health-nutrition-andpopulation-statistics (accessed March 10, 2017).

8 Kengne AP, June-Rose McHiza Z, Amoah AG, Mbanya JC. Cardiovascular diseases and diabetes as economic and developmental challenges in Africa. Prog Cardiovasc Dis 2013; 56: 302-13.

9 Institute for Health Metrics and Evaluation. Global burden of disease (GBD). http://www.healthdata.org/gbd (accessed March 10, 2017).

10 Levitt NS. Diabetes in Africa: epidemiology, management and healthcare challenges. Heart 2008; 94: 1376-82.

11 Manne-Goehler J, Atun R, Stokes A, et al. Diabetes diagnosis and care in sub-Saharan Africa: pooled analysis of individual data from 12 countries. Lancet Diabetes Endocrinol 2016; 4: 903-12.

12 World Bank. World development indicators. 2016. http://data. worldbank.org/products/wdi (accessed April 27, 2016).

13 Allen L. Non-communicable disease funding. Lancet Diabetes Endocrinol 2017; 5: 92.

14 Dieleman JL, Schneider MT, Haakenstad A, et al. Development assistance for health: past trends, associations, and the future of international financial flows for health. Lancet 2016; 387: 2536-44.

15 UN. High-level meeting on non-communicable diseases. http://www.un.org/en/ga/president/65/issues/ncdiseases.shtml (accessed March 10, 2017).

16 NCD Risk Factor Collaboration (NCD-RisC). Worldwide trends in diabetes since 1980: a pooled analysis of 751 population-based studies with 4.4 million participants. Lancet 2016; 387: 1513-30.

17 Selvin E, Rawlings AM, Grams M, et al. Fructosamine and glycated albumin for risk stratification and prediction of incident diabetes and microvascular complications: a prospective cohort analysis of the Atherosclerosis Risk in Communities (ARIC) study. Lancet Diabetes Endocrinol 2014; 2: 279-88.

18 Warren B, Pankow JS, Matsushita K, et al. Comparative prognostic performance of definitions of prediabetes: a prospective cohort analysis of the Atherosclerosis Risk in Communities (ARIC) study. Lancet Diabetes Endocrinol 2017; 5: 34-42.

19 NCD Risk Factor Collaboration (NCD-RisC). Effects of diabetes definition on global surveillance of diabetes prevalence and diagnosis: a pooled analysis of 96 population-based studies with 331288 participants. Lancet Diabetes Endocrinol 2015; 3: 624-37.

20 Werfalli M, Engel ME, Musekiwa A, Kengne AP, Levitt NS. The prevalence of type 2 diabetes among older people in Africa: a systematic review. Lancet Diabetes Endocrinol 2016; 4: 72-84.

21 Tian Y, Jiang C, Wang M, et al. BMI, leisure-time physical activity, and physical fitness in adults in China: results from a series of national surveys, 2000-14. Lancet Diabetes Endocrinol 2016; 4: 487-97.

22 Zimmet P, Alberti KG, Magliano DJ, Bennett PH. Diabetes mellitus statistics on prevalence and mortality: facts and fallacies. Nat Rev Endocrinol 2016; 12: 616-22.

23 Bennett PH, Magliano DJ, Alberti KG, Zimmet P. Liberating non-communicable disease data. Lancet Diabetes Endocrinol 2016; 4: 815-16.

24 Davies J, Yudkin JS, Atun R. Liberating data: the crucial weapon in the fight against NCDs. Lancet Diabetes Endocrinol 2016; 4: 197-98.

25 IDF. IDF Diabetes Atlas, 7th edition. Brussels: International Diabetes Federation, 2015. http://www.diabetesatlas.org/ (accessed March 10, 2017).

26 Hall V, Thomsen RW, Henriksen O, Lohse N. Diabetes in Sub Saharan Africa 1999-2011: epidemiology and public health implications. A systematic review. BMC Public Health 2011; 11: 564.

27 Scott A, Ejikeme CS, Clottey EN, Thomas JG. Obesity in sub-Saharan Africa: development of an ecological theoretical framework. Health Promot Int 2013; 28: 4-16.
28 McCarthy MI. Genomics, type 2 diabetes, and obesity. N Engl J Med 2010; 363: 2339-50.

29 Helgason A, Pálsson S, Thorleifsson G, et al. Refining the impact of TCF7L2 gene variants on type 2 diabetes and adaptive evolution. Nat Genet 2007; 39: 218-25.

30 Adeyemo AA, Tekola-Ayele F, Doumatey AP, et al. Evaluation of genome wide association study associated type 2 diabetes susceptibility loci in sub Saharan Africans. Front Genet 2015; 6: 335.

31 Yako YY, Guewo-Fokeng M, Balti EV, et al. Genetic risk of type 2 diabetes in populations of the African continent: a systematic review and meta-analyses. Diabetes Res Clin Pract 2016; 114: 136-50.

32 Corona E. Mapping genetic risk of disease across the world. 2016. http://geneworld.erikcorona.com/geneworld (accessed March 10, 2017).

33 Marshall MC Jr. Diabetes in African Americans. Postgrad Med J 2005; 81: 734-40.

34 CDC. National diabetes fact sheet: national estimates and general information on diabetes and prediabetes in the United States. Atlanta: US Department of Health and Human Services, 2011.

35 Zimmet PZ, Magliano DJ, Herman WH, Shaw JE. Diabetes: a 21st century challenge. Lancet Diabetes Endocrinol 2014; 2: 56-64.

36 Noble JA, Johnson J, Lane JA, Valdes AM. HLA class II genotyping of African American type 1 diabetic patients reveals associations unique to African haplotypes. Diabetes 2013; 62: 3292-99.

37 Mbanya JCN, Motala AA, Sobngwi E, Assah FK, Enoru ST. Diabetes in sub-Saharan Africa. Lancet 2010; 375: 2254-66.

38 Kengne AP, Echouffo-Tcheugui JB, Sobngwi E, Mbanya JC. New insights on diabetes mellitus and obesity in Africa-part 1: prevalence, pathogenesis and comorbidities. Heart 2013; 99: 979-83.

39 O'Hara EG, Nuche-Berenguer B, Kirui NK, et al. Diabetes in rural Africa: what can Kenya show us? Lancet Diabetes Endocrinol 2016; 4: 807-09.

40 NCD Risk Factor Collaboration (NCD-RisC). Trends in adult bodymass index in 200 countries from 1975 to 2014: a pooled analysis of 1698 population-based measurement studies with $19 \cdot 2$ million participants. Lancet 2016; 387: 1377-96.

41 Monteiro CA, D’A Benicio MH, Conde WL, Popkin BM. Shifting obesity trends in Brazil. Eur J Clin Nutr 2000; 54: 342-46.

42 Ziraba AK, Fotso JC, Ochako R. Overweight and obesity in urban Africa: a problem of the rich or the poor? BMC Public Health 2009; 9: 465 .

43 Popkin BM, Adair LS, Ng SW. Global nutrition transition and the pandemic of obesity in developing countries. Nutr Rev 2012; 70: 3-21.

44 IFAD. Structural and rural transformation in Africa. In: Rural Development Report 2016. Rome: Quintily, 2016.

45 Pampel FC, Denney JT, Krueger PM. Obesity, SES, and economic development: a test of the reversal hypothesis. Soc Sci Med 2012; 74: $1073-81$.

46 Aitsi-Selmi A, Bell R, Shipley MJ, Marmot MG. Education modifies the association of wealth with obesity in women in middle-income but not low-income countries: an interaction study using seven national datasets, 2005-2010. PLoS One 2014; 9: e90403.

47 Murphy GA, Asiki G, Ekoru K, et al. Sociodemographic distribution of non-communicable disease risk factors in rural Uganda: a cross-sectional study. Int J Epidemiol 2013; 42: 1740-53.

48 Gillman MW. Prenatal famine and developmental origins of type 2 diabetes. Lancet Diabetes Endocrinol 2015; 3: 751-52.

49 Lelijveld N, Seal A, Wells JC, et al. Chronic disease outcomes after severe acute malnutrition in Malawian children (ChroSAM): a cohort study. Lancet Glob Health 2016; 4: e654-62.

50 Gill GV, Mbanya JC, Ramaiya KL, Tesfaye S. A sub-Saharan African perspective of diabetes. Diabetologia 2009; 52: 8-16.

51 UN, Department of Economic and Social Affairs, Population Division. World fertility report 2013: fertility at the extremes. 2014. http://www.un.org/en/development/desa/population/ publications/pdf/fertility/worldFertilityReport2013.pdf (accessed March 10, 2017).

52 Geldsetzer P, Manne-Goehler J, Bärnighausen T, Davies JI. What research is needed to address the co-epidemics of HIV and cardiometabolic disease in sub-Saharan Africa? Lancet Diabetes Endocrinol 2017; published online March 17. DOI:http://dx.doi. org/10.1016/S2213-8587(17)30091-8. 
53 Alemu S, Dessie A, Seid E, et al. Insulin-requiring diabetes in rural Ethiopia: should we reopen the case for malnutrition-related diabetes? Diabetologia 2009; 52: 1842-45.

54 Gale EAM. The rise of childhood type 1 diabetes in the 20th century. Diabetes 2002; 51: 3353-61.

55 Zung A, Elizur M, Weintrob N, et al. Type 1 diabetes in Jewish Ethiopian immigrants in Israel: HLA class II immunogenetics and contribution of new environment. Hum Immunol 2004; 65: 1463-68.

56 Marshall SL, Edidin D, Sharma V, Ogle G, Arena VC, Orchard T. Current clinical status, glucose control, and complication rates of children and youth with type 1 diabetes in Rwanda. Pediatr Diabetes 2013; 14: 217-26.

57 Karvonen M, Viik-Kajander M, Moltchanova E, Libman I, LaPorte R, Tuomilehto J. Incidence of childhood type 1 diabetes worldwide. Diabetes Mondiale (DiaMond) Project Group. Diabetes Care 2000; 23: 1516-26.

58 Piloya-Were T, Sunni M, Ogle GD, Moran A. Childhood diabetes in Africa. Curr Opin Endocrinol Diabetes Obes 2016; 23: 306-11.

59 Rwiza HT, Swai ABM, McLarty DG. Failure to diagnose diabetic ketoacidosis in Tanzania. Diabet Med 1986; 3: 181-83.

60 Ogle GD, Middlehurst AC, Silink M. The IDF Life for a Child Program Index of diabetes care for children and youth. Pediatr Diabetes 2016; 17: 374-84.

61 Marshall SL, Edidin D, Arena VC, et al. Prevalence and incidence of clinically recognized cases of type 1 diabetes in children and adolescents in Rwanda, Africa. Diabet Med 2015; 32: 1186-92.

62 Marshall SL, Edidin DV, Arena VC, et al. Glucose control in Rwandan youth with type 1 diabetes following establishment of systematic, $\mathrm{HbA}_{\mathrm{cc}}$ based, care and education. Diabetes Res Clin Pract 2015; 107: 113-22.

63 Umpierrez GE, Smiley D, Kitabchi AE. Narrative review: ketosisprone type 2 diabetes mellitus. Ann Intern Med 2006; 144: 350-57.

64 Ma RCW, Schmidt MI, Tam WH, McIntyre HD, Catalano PM. Clinical management of pregnancy in the obese mother: before conception, during pregnancy, and post partum. Lancet Diabetes Endocrinol 2016; 4: 1037-49.

65 Poston L, Caleyachetty R, Cnattingius S, et al. Preconceptional and maternal obesity: epidemiology and health consequences. Lancet Diabetes Endocrinol 2016; 4: 1025-36.

66 World Obesity Federation. World map of obesity. 2015. http://www. worldobesity.org/resources/world-map-obesity (accessed March 10, 2017).

67 Macaulay S, Dunger DB, Norris SA. Gestational diabetes mellitus in Africa: a systematic review. PLoS One 2014; 9: e97871.

68 Olagbuji BN, Atiba AS, Olofinbiyi BA, et al, for the Gestational Diabetes Study Group-Nigeria. Prevalence of and risk factors for gestational diabetes using 1999, 2013 WHO and IADPSG criteria upon implementation of a universal one-step screening and diagnostic strategy in a sub-Saharan African population. Eur J Obstet Gynecol Reprod Biol 2015; 189: 27-32.

69 WHO, Department of Noncommunicable Disease Surveillance. Definition, diagnosis and classification of diabetes mellitus and its complications: report of a WHO consultation. Part 1 , Diagnosis and classification of diabetes mellitus. Geneva: World Health Organization, 1999.

70 Colagiuri S, Falavigna M, Agarwal MM, et al. Strategies for implementing the WHO diagnostic criteria and classification of hyperglycaemia first detected in pregnancy. Diabetes Res Clin Pract 2014; 103: 364-72.

71 Metzger BE, Gabbe SG, Persson B, et al, for the International Association of Diabetes and Pregnancy Study Groups Consensus Panel. International association of diabetes and pregnancy study groups recommendations on the diagnosis and classification of hyperglycemia in pregnancy. Diabetes Care 2010; 33: 676-82.

72 Metzger BE, Lowe LP, Dyer AR, et al, for the HAPO Study Cooperative Research Group. Hyperglycemia and adverse pregnancy outcomes. N Engl J Med 2008; 358: 1991-2002.

73 Farrar D, Fairley L, Santorelli G, et al. Association between hyperglycaemia and adverse perinatal outcomes in south Asian and white British women: analysis of data from the Born in Bradford cohort. Lancet Diabetes Endocrinol 2015; 3: 795-804.

74 Hedderson M, Ehrlich S, Sridhar S, Darbinian J, Moore S, Ferrara A. Racial/ethnic disparities in the prevalence of gestational diabetes mellitus by BMI. Diabetes Care 2012; 35: 1492-98.
75 Morrison JL, Hodgson LAB, Lim LL, Al-Qureshi S. Diabetic retinopathy in pregnancy: a review. Clin Experiment Ophthalmo 2016; 44: 321-34.

76 Inkster ME, Fahey TP, Donnan PT, Leese GP, Mires GJ, Murphy DJ. Poor glycated haemoglobin control and adverse pregnancy outcomes in type 1 and type 2 diabetes mellitus: systematic review of observational studies. BMC Pregnancy Childbirth 2006; 6: 30.

77 Fraser RB. The fate of the pregnant diabetic in the developing country: Kenya. Diabetologia 1982; 22: 21-24.

78 Huddle KR. Audit of the outcome of pregnancy in diabetic women in Soweto, South Africa, 1992-2002. S Afr Med J 2005; 95: 789-94.

79 Zeck W, McIntyre HD. Gestational diabetes in rural East Africa: a call to action. J Womens Health (Larchmt) 2008; 17: 403-11.

80 Coetzee EJ, Jackson WPU. The management of non-insulindependent diabetes during pregnancy. Diabetes Res Clin Pract 1985-1986; 1: 281-87.

81 Gill GV, Huddle KRL, Monkoe G. Long-term (20 years) outcome and mortality of type 1 diabetic patients in Soweto, South Africa. Diabet Med 2005; 22: 1642-46.

82 Ekpebegh CO, Coetzee EJ, van der Merwe L, Levitt NS. A 10-year retrospective analysis of pregnancy outcome in pregestational type 2 diabetes: comparison of insulin and oral glucose-lowering agents. Diabet Med 2007; 24: 253-58.

83 WHO. National capacity to address and respond to NCDs: existence of operational policies, strategies, or action plans. 2016. http://www. who.int/gho/ncd/health_system_response/policy_text/en (accessed March 10, 2017).

84 Riza AL, Pearson F, Ugarte-Gil C, et al. Clinical management of concurrent diabetes and tuberculosis and the implications for patient services. Lancet Diabetes Endocrinol 2014; 2: 740-53.

85 van Crevel R, van de Vijver S, Moore DAJ. The global diabetes epidemic: what does it mean for infectious diseases in tropical countries? Lancet Diabet Endocrinol 2016; 5: 457-68.

86 Pearson-Stuttard J, Blundell S, Harris T, Cook DG, Critchley J. Diabetes and infection: assessing the association with glycaemic control in population-based studies. Lancet Diabetes Endocrinol 2016 4: $148-58$.

87 Nou E, Lo J, Hadigan C, Grinspoon SK. Pathophysiology and management of cardiovascular disease in patients with HIV. Lancet Diabetes Endocrinol 2016; 4: 598-610.

88 Sankoh O, Byass P. Cause-specific mortality at INDEPTH Health and Demographic Surveillance System Sites in Africa and Asia: concluding synthesis. Glob Health Action 2014; 7: 25590.

89 I SATHL-AIDMTAC. Le diabète juvénile au Mali. Rev Fr Endocrinol Clin 1999; 40: 513-21.

90 Beran D, Yudkin JS, de Courten M. Access to care for patients with insulin-requiring diabetes in developing countries: case studies of Mozambique and Zambia. Diabetes Care 2005; 28: 2136-40.

91 Livingstone SJ, Levin D, Looker HC, et al, for the Scottish Diabetes Research Network epidemiology group, and the Scottish Renal Registry. Estimated life expectancy in a Scottish cohort with type 1 diabetes, 2008-2010. JAMA 2015; 313: 37-44.

92 Bertram MY, Jaswal AV, Van Wyk VP, Levitt NS, Hofman KJ. The non-fatal disease burden caused by type 2 diabetes in South Africa, 2009. Glob Health Action 2013; 6: 19244.

93 Cheung N, Mitchell P, Wong TY. Diabetic retinopathy. Lancet 2010; 376: $124-36$.

94 Bourne RR, Stevens GA, White RA, et al, for the Vision Loss Expert Group. Causes of vision loss worldwide, 1990-2010: a systematic analysis. Lancet Glob Health 2013; 1: e339-49.

95 Burgess PI, MacCormick IJC, Harding SP, Bastawrous A Beare NAV, Garner P. Epidemiology of diabetic retinopathy and maculopathy in Africa: a systematic review. Diabet Med 2013; 30: $399-412$.

96 Lee R, Wong TY, Sabanayagam C. Epidemiology of diabetic retinopathy, diabetic macular edema and related vision loss. Eye Vis 2015; $2: 17$.

97 Global Burden of Metabolic Risk Factors for Chronic Diseases Collaboration. Cardiovascular disease, chronic kidney disease, and diabetes mortality burden of cardiometabolic risk factors from 1980 to 2010: a comparative risk assessment. Lancet Diabetes Endocrinol 2014; 2: 634-47. 
98 Global Burden of Disease Study 2013 Collaborators. Global, regional, and national incidence, prevalence, and years lived with disability for 301 acute and chronic diseases and injuries in 188 countries, 1990-2013: a systematic analysis for the Global Burden of Disease Study 2013. Lancet 2015; 386: 743-800.

99 Stanifer JW, Jing B, Tolan S, et al. The epidemiology of chronic kidney disease in sub-Saharan Africa: a systematic review and meta-analysis. Lancet Glob Health 2014; 2: e174-81.

100 Lancet Diabetes Endocrinology. Diabetic kidney disease: what does the next era hold? Lancet Diabetes Endocrinol 2015; 3: 665.

101 Noubiap JJ, Naidoo J, Kengne AP. Diabetic nephropathy in Africa: a systematic review. World J Diabetes 2015; 6: 759-73.

102 Genovese G, Friedman DJ, Ross MD, et al. Association of trypanolytic ApoL1 variants with kidney disease in African Americans. Science 2010; 329: 841-45.

103 Breyer M. Faculty of 1000 evaluation for association of trypanolytic ApoL1 variants with kidney disease in African Americans. F1000Post-publication peer review of the biomedical literature: Faculty of 1000, Ltd.

104 Osafo C, Raji YR, Olanrewaju T, et al, for the H3Africa Kidney Disease Research Network. Genomic approaches to the burden of kidney disease in Sub-Saharan Africa: the Human Heredity and Health in Africa (H3Africa) Kidney Disease Research Network. Kidney Int 2016; 90: 2-5.

105 Abbas ZG. The global burden of diabetic foot. In: Pendsey S, ed. Contemporary management of the diabetic foot. New Delhi: Jaypee Brothers Medical Publishers, 2014: 24-30.

106 Margolis DJ, Malay DS, Hoffstad OJ, et al. Prevalence of diabetes, diabetic foot ulcer, and lower extremity amputation among Medicare beneficiaries, 2006 to 2008. Data Points Publication Series, 2011.

107 Bakker K, Apelqvist J, Lipsky BA, Van Netten JJ, for the International Working Group on the Diabetic Foot. The 2015 IWGDF guidance documents on prevention and management of foot problems in diabetes: development of an evidence-based globa consensus. Diabetes Metab Res Rev 2016; 32 (suppl 1): 2-6.

108 Cockburn N, Steven D, Lecuona K, et al. Prevalence, causes and socio-economic determinants of vision loss in Cape Town, South Africa. PLoS One 2012; 7: e30718.

109 Abbas ZG, Lutale JK, Bakker K, Baker N, Archibald LK. The 'Step by Step' Diabetic Foot Project in Tanzania: a model for improving patient outcomes in less-developed countries. Int Wound J 2011; 8: 169-75.

110 Ndip EA, Tchakonte B, Mbanya JC. A study of the prevalence and risk factors of foot problems in a population of diabetic patients in Cameroon. Int J Low Extrem Wounds 2006; 5: 83-88.

111 Ogbera AO, Fasanmade O, Ohwovoriole AE, Adediran O. An assessment of the disease burden of foot ulcers in patients with diabetes mellitus attending a teaching hospital in Lagos, Nigeria. Int J Low Extrem Wounds 2006; 5: 244-49.

112 Tesfaye S, Boulton AJM, Dyck PJ, et al, for the Toronto Diabetic Neuropathy Expert Group. Diabetic neuropathies: update on definitions, diagnostic criteria, estimation of severity, and treatments. Diabetes Care 2010; 33: 2285-93.

113 Abbas ZG, Archibald LK. Challenges for management of the diabetic foot in Africa: doing more with less. Int Wound J 2007; 4: 305-13.

114 Abbas ZG, Lutale JK, Archibald LK. Diabetic foot ulcers and ethnicity in Tanzania: a contrast between African and Asian populations. Int Wound J 2009; 6: 124-31.

115 Jeffcoate WJ, Harding KG. Diabetic foot ulcers. Lancet 2003; 361: $1545-51$

116 Levitt NS, Bradshaw D, Zwarenstein MF, Bawa AA, Maphumolo S. Audit of public sector primary diabetes care in Cape Town, South Africa: high prevalence of complications, uncontrolled hyperglycaemia, and hypertension. Diabet Med 1997; 14: 1073-77.

117 Okello S, Millard A, Owori R, et al. Prevalence of lower extremity peripheral artery disease among adult diabetes patients in southwestern Uganda. BMC Cardiovasc Disord 2014; 14: 75.

118 Kumar A, Mash B, Rupesinghe G. Peripheral arterial disease-high prevalence in rural black South Africans. S Afr Med J 2007; 97: 285-88.

119 Abbas ZG, Lutale J, Archibald LK. Rodent bites on the feet of diabetes patients in Tanzania. Diabet Med 2005; 22: 631-33.
120 Dunbar GL, Hellenberg DA, Levitt NS. Diabetes mellitus and non-traumatic lower extremity amputations in four public sector hospitals in Cape Town, South Africa, during 2009 and 2010. S Afr Med J 2015; 105: 1053-56.

121 Gulam-Abbas Z, Lutale JK, Morbach S, Archibald LK. Clinical outcome of diabetes patients hospitalized with foot ulcers, Dar es Salaam, Tanzania. Diabet Med 2002; 19: 575-79.

122 Lam C, Martinson N, Hepp L, et al. Prevalence of tobacco smoking in adults with tuberculosis in South Africa. Int J Tuberc Lung Dis 2013; 17: 1354-57.

123 Waweru P, Anderson R, Steel H, Venter WD, Murdoch D, Feldman C. The prevalence of smoking and the knowledge of smoking hazards and smoking cessation strategies among HIV-positive patients in Johannesburg, South Africa. S Afr Med J 2013; 103: 858-60.

124 Gaziano TA. Cardiovascular disease in the developing world and its cost-effective management. Circulation 2005; 112: 3547-53.

125 Addo J, Smeeth L, Leon DA. Hypertension in sub-Saharan Africa: a systematic review. Hypertension 2007; 50: 1012-18.

126 Gudina EK, Amade ST, Tesfamichael FA, Ram R. Assessment of quality of care given to diabetic patients at Jimma University Specialized Hospital diabetes follow-up clinic, Jimma, Ethiopia. BMC Endocr Disord 2011; 11: 19.

127 Adeniyi OV, Yogeswaran P, Longo-Mbenza B, Ter Goon D. Uncontrolled hypertension and its determinants in patients with concomitant type 2 diabetes mellitus (T2DM) in rural South Africa. PLoS One 2016; 11: e0150033.

128 Sobngwi E, Ndour-Mbaye M, Boateng KA, et al. Type 2 diabetes control and complications in specialised diabetes care centres of six sub-Saharan African countries: the Diabcare Africa study. Diabetes Res Clin Pract 2012; 95: 30-36.

129 Kengne AP, Sobngwi E, Echouffo-Tcheugui JB, Mbanya JC. New insights on diabetes mellitus and obesity in Africa-Part 2: prevention, screening and economic burden. Heart 2013; 99: 1072-77.

130 Kengne AP, Limen SN, Sobngwi E, Djouogo CF, Nouedoui C. Metabolic syndrome in type 2 diabetes: comparative prevalence according to two sets of diagnostic criteria in sub-Saharan Africans. Diabetol Metab Syndr 2012; 4: 22.

131 Hertz JT, Reardon JM, Rodrigues CG, et al. Acute myocardial infarction in sub-Saharan Africa: the need for data. PLoS One 2014; 9: e96688.

132 Kengne AP, Amoah AG, Mbanya JC. Cardiovascular complications of diabetes mellitus in sub-Saharan Africa. Circulation 2005 112: 3592-601.

133 Feigin VL, Lawes CMM, Bennett DA, Barker-Collo SL, Parag V. Worldwide stroke incidence and early case fatality reported in 56 population-based studies: a systematic review. Lancet Neurol 2009; 8: 355-69.

134 Lekoubou A, Nkoke C, Dzudie A, Kengne AP. Stroke admission and case-fatality in an urban medical unit in sub-Saharan Africa: a fourteen year trend study from 1999 to 2012. J Neurol Sci 2015; 350: 24-32.

135 Echouffo-Tcheugui JB, Dzudie A, Epacka ME, et al. Prevalence and determinants of undiagnosed diabetes in an urban sub-Saharan African population. Prim Care Diabetes 2012; 6: 229-34.

136 American Diabetes Association. Standards of medical care in diabetes-2014. Diabetes Care 2014; 37 (suppl 1): S14-80.

137 American Diabetes Association. Standards of medical care in diabetes-2017: summary of revisions. Diabetes Care 2017; 40 (suppl 1): S4-5.

138 World Bank. Service Delivery Indicators. http://datatopics. worldbank.org/sdi (accessed March 10, 2017).

139 Beran D, Yudkin JS. Looking beyond the issue of access to insulin: what is needed for proper diabetes care in resource poor settings. Diabetes Res Clin Pract 2010; 88: 217-21.

140 Swai AB, Lyimo PJ, Rutayuga F, McLarty DG. Diabetes mellitus misdiagnosed as AIDS. Lancet 1989; 2: 976

141 Makani J, Matuja W, Liyombo E, Snow RW, Marsh K, Warrell DA. Admission diagnosis of cerebral malaria in adults in an endemic area of Tanzania: implications and clinical description. QJM 2003; 96: 355-62.

142 Majaliwa ES, Munubhi E, Ramaiya K, et al. Survey on acute and chronic complications in children and adolescents with type 1 diabetes at Muhimbili National Hospital in Dar es Salaam, Tanzania. Diabetes Care 2007; 30: 2187-92. 
143 Folb N, Timmerman V, Levitt NS, et al. Multimorbidity, control and treatment of noncommunicable diseases among primary healthcare attenders in the Western Cape, South Africa. S Afr Med J 2015; 105: 642-47.

144 Mash RJ, Rhode H, Zwarenstein M, et al. Effectiveness of a group diabetes education programme in under-served communities in South Africa: a pragmatic cluster randomized controlled trial. Diabet Med 2014; 31: 987-93.

145 Chinenye S, Uloko AE, Ogbera AO, et al. Profile of Nigerians with diabetes mellitus-Diabcare Nigeria study group (2008): results of a multicenter study. Indian J Endocrinol Metab 2012; 16: 558-64.

146 Ogle GD, Kim H, Middlehurst AC, Silink M, Jenkins AJ. Financial costs for families of children with type 1 diabetes in lower-income countries. Diabet Med 2016; 33: 820-26.

147 Spurling GK, Mansfield PR, Montgomery BD, et al. Information from pharmaceutical companies and the quality, quantity, and cost of physicians' prescribing: a systematic review. PLoS Med 2010; 7: e1000352.

148 Wu JHY, Foote C, Blomster J, et al. Effects of sodium-glucose cotransporter-2 inhibitors on cardiovascular events, death, and major safety outcomes in adults with type 2 diabetes: a systematic review and meta-analysis. Lancet Diabetes Endocrinol 2016; 4: 411-19.

149 Beran D, Ewen M, Laing R. Constraints and challenges in access to insulin: a global perspective. Lancet Diabetes Endocrinol 2016; 4: 275-85.

150 Ogle GD, Abdullah M, Mason D, Januszewski AS, Besançon S. Insulin storage in hot climates without refrigeration: temperature reduction efficacy of clay pots and other techniques. Diabet Med 2016; 33: 1544-53.

151 Majaliwa ES, Elusiyan BE, Adesiyun OO, et al. Type 1 diabetes mellitus in the African population: epidemiology and management challenges. Acta Biomed 2008; 79: 255-59.

152 Basu S, Shankar V, Yudkin JS. Comparative effectiveness and cost-effectiveness of treat-to-target versus benefit-based tailored treatment of type 2 diabetes in low-income and middle-income countries: a modelling analysis. Lancet Diabetes Endocrinol 2016; 4: 922-32.

153 Gaede P, Lund-Andersen H, Parving HH, Pedersen O. Effect of a multifactorial intervention on mortality in type 2 diabetes. N Engl J Med 2008; 358: 580-91.

154 Werner ME, van de Vijver S, Adhiambo M, Egondi T, Oti SO, Kyobutungi C. Results of a hypertension and diabetes treatmen program in the slums of Nairobi: a retrospective cohort study. BMC Health Serv Res 2015; 15: 512.

155 Adedeji AR, Tumbo J, Govender I. Adherence of doctors to a clinical guideline for hypertension in Bojanala district, North-West Province, South Africa. Afr J Prim Health Care Fam Med 2015; 7: 776.

156 Kengne AP, Njamnshi AK, Mbanya JC. Cardiovascular risk reduction in diabetes in sub-Saharan Africa: what should the priorities be in the absence of global risk evaluation tools? Clin Med Insights Cardiol 2008; 2: 25-31.

157 Ataklte F, Erqou S, Kaptoge S, Taye B, Echouffo-Tcheugui JB Kengne AP. Burden of undiagnosed hypertension in sub-Saharan Africa: a systematic review and meta-analysis. Hypertension 2015; 65: 291-98.

158 Yusuf S, Wood D, Ralston J, Reddy KS. The World Heart Federation's vision for worldwide cardiovascular disease prevention Lancet 2015; 386: 399-402.

159 Cleland CR, Burton MJ, Hall C, et al. Diabetic retinopathy screening: experiences from northern Tanzania. Lancet Diabetes Endocrinol 2016; 4: 10-12.

160 Cleland CR, Burton MJ, Hall C, et al. Diabetic retinopathy in Tanzania: prevalence and risk factors at entry into a regional screening programme. Trop Med Int Health 2016; 21: 417-26.

161 Burgess PI, Msukwa G, Beare NAV. Diabetic retinopathy in sub-Saharan Africa: meeting the challenges of an emerging epidemic. BMC Med 2013; 11: 157

162 Matimba A, Woodward R, Tambo E, Ramsay M, Gwanzura L, Guramatunhu S. Tele-ophthalmology: opportunities for improving diabetes eye care in resource- and specialist-limited sub-Saharan African countries. J Telemed Telecare 2016; 22: 311-16.

163 Bastawrous A, Rono HK, Livingstone IAT, et al. Development and validation of a smartphone-based visual acuity test (peek acuity) for clinical practice and community-based fieldwork. JAMA Ophthalmol 2015; 133: 930-37.
164 Naicker S. Burden of end-stage renal disease in sub-Saharan Africa. Clin Nephrol 2010; 74 (suppl 1): S13-16.

165 Das P, Horton R. Physical activity-time to take it seriously and regularly. Lancet 2016; 388: 1254-55.

166 Griffin SJ, Borch-Johnsen K, Davies MJ, et al. Effect of early intensive multifactorial therapy on 5-year cardiovascular outcomes in individuals with type 2 diabetes detected by screening (ADDITION-Europe): a cluster-randomised trial. Lancet 2011; 378: 156-67.

167 Durão S, Ajumobi O, Kredo T, et al. Evidence insufficient to confirm the value of population screening for diabetes and hypertension in low- and-middle-income settings. S Afr Med J 2015; 105: 98-102.

168 Echouffo-Tcheugui JB, Mayige M, Ogbera AO, Sobngwi E, Kengne AP. Screening for hyperglycemia in the developing world: rationale, challenges and opportunities. Diabetes Res Clin Pract 2012; 98: 199-208.

169 Dalal S, Beunza JJ, Volmink J, et al. Non-communicable diseases in sub-Saharan Africa: what we know now. Int J Epidemiol 2011; 40: 885-901.

170 Atun R, Aydın S, Chakraborty S, et al. Universal health coverage in Turkey: enhancement of equity. Lancet 2013; 382: 65-99.

171 WHO. Global Health Observatory country views. 2016. http://apps. who.int/gho/data/node.country (accessed March 10, 2017).

172 WHO. Global Health Observatory data repository. Polices, strategies, and action plans: data by country. 2016. http://apps.who.int/gho/ data/node.main.A907?lang=en (accessed April 13, 2016).

173 Meheus F, McIntyre D. Fiscal space for domestic funding of health and other social services. Health Econ Policy Law 2017; 12: 159-77.

174 UN. Abuja declaration on HIV/AIDS, tuberculosis, and other infectious diseases. 2001. http://www.un.org/ga/aids/pdf/abuja_ declaration.pdf (accessed June 12, 2017).

175 Huffman MD, Rao KD, Pichon-Riviere A, et al. A cross-sectional study of the microeconomic impact of cardiovascular disease hospitalization in four low- and middle-income countries. PLoS One 2011; 6: e20821.

176 Wang Q, Brenner S, Leppert G, Banda TH, Kalmus O, De Allegri M. Health seeking behaviour and the related household out-of-pocket expenditure for chronic non-communicable diseases in rural Malawi. Health Policy Plan 2015; 30: 242-52.

177 Wang Q, Fu AZ, Brenner S, Kalmus O, Banda HT, De Allegri M. Out-of-pocket expenditure on chronic non-communicable diseases in sub-Saharan Africa: the case of rural Malawi. PLoS One 2015; 10: e0116897.

178 Global Health Workforce Allicance, WHO. A universal truth: no health without a workforce. Geneva: World Health Organization, 2013.

179 Mullan F, Frehywot S, Omaswa F, et al. Medical schools in sub-Saharan Africa. Lancet 2011; 377: 1113-21.

180 Scheffler RM, Mahoney CB, Fulton BD, Dal Poz MR, Preker AS. Estimates of health care professional shortages in sub-Saharan Africa by 2015. Health Aff 2009; 28: w849-62.

181 Mullan F, Frehywot S. Non-physician clinicians in 47 sub-Saharan African countries. Lancet 2007; 370: 2158-63.

182 Celletti F, Wright A, Palen J, et al. Can the deployment of community health workers for the delivery of HIV services represent an effective and sustainable response to health workforce shortages? Results of a multicountry study. AIDS 2010; 24 (suppl 1): S45-57.

183 Mwai GW, Mburu G, Torpey K, Frost P, Ford N, Seeley J. Role and outcomes of community health workers in HIV care in sub Saharan Africa: a systematic review. J Int AIDS Soc 2013; 16: 18586

184 WHO. Service Availability and Readiness Assessment (SARA). http://www.who.int/healthinfo/systems/sara_methods/en (accessed March 10, 2017).

185 Beran D, Silva Matos C, Yudkin JS. The Diabetes UK Mozambique Twinning Programme. Results of improvements in diabetes care in Mozambique: a reassessment 6 years later using the Rapid Assessment Protocol for Insulin Access. Diabet Med 2010; 27: 855-61.

186 Bowser D, Sparkes SP, Mitchell A, et al. Global Fund investments in human resources for health: innovation and missed opportunities for health systems strengthening. Health Policy Plan 2014; 29: 986-97.

187 WHO. WHO global action plan for the prevention and control of NCDs 2013-2020. Geneva: World Health Organization, 2013. 
188 Peck R, Mghamba J, Vanobberghen F, et al. Preparedness of Tanzanian health facilities for outpatient primary care of hypertension and diabetes: a cross-sectional survey. Lancet Glob Health 2014; 2: e285-92.

189 Cameron A, Ewen M, Ross-Degnan D, Ball D, Laing R. Medicine prices, availability, and affordability in 36 developing and middle-income countries: a secondary analysis. Lancet 2009; 373: 240-49.

190 Mendis S, Fukino K, Cameron A, et al. The availability and affordability of selected essential medicines for chronic diseases in six low- and middle-income countries. Bull World Health Organ 2007; 85: 279-88.

191 Kaplan W, Sharma A, Kolaczyk E, Shappell H. Insulin trade profile. April 2016. http://haiweb.org/wp-content/uploads/2016/06/ ACCISS-TradeReport_FINAL_2.pdf (accessed April 10, 2016).

192 MSH. International medical products price guide. 2015 http://erc.msh.org/mainpage. cfm?file $=1.0 . h$ tm\&module $=$ dmp\&language $=$ english $($ accessed March 10, 2017).

193 Ewen M, Joosse H-J, Ashigbie P, Beran D, Laing R. Insulin prices profile. April 2016. http://haiweb.org/wp-content/uploads/2016/04/ ACCISS-Prices-report_FINAL-1.pdf (accessed April 10, 2016).

194 WHO. Drugs used in diabetes. 2013. http://www.whocc.no/atc_ ddd_index/?code=A10A (accessed March 10, 2017).

195 World Bank. PovcalNet: an online analysis tool for global poverty monitoring. http://iresearch.worldbank.org/PovcalNet/home.aspx (accessed June 23, 2017)

196 World Bank. Uganda-Service Delivery Indicators Health Survey 2013 - harmonized public use data. http://microdata.worldbank. org/index.php/catalog/2750/sampling (accessed June 10, 2017).

197 Tudor Car L, Brusamento S, Elmoniry H, et al. The uptake of integrated perinatal prevention of mother-to-child HIV transmission programs in low- and middle-income countries: a systematic review. PLoS One 2013; 8: e56550.

198 Haber N, Pillay D, Porter K, Bärnighausen T. Constructing the cascade of HIV care: methods for measurement. Curr Opin HIV AIDS 2016; 11: 102-08.

199 Ali MK, Bullard KM, Gregg EW, Del Rio C. A cascade of care for diabetes in the United States: visualizing the gaps. Ann Intern Med 2014; 161: 681-89.

200 WHO. The STEPS instrument and support materials. 2015. http://www.who.int/chp/steps/instrument/en (accessed March 10, 2017).

201 Ministry of Health and Social Services, Namibia Statistics Agency. Namibia demographic and health survey 2013. 2014. https://dhsprogram.com/pubs/pdf/FR298/FR298.pdf (accessed March 10, 2017).

202 Labadarios D, Shisana O, Rehle T, Simbayi L. SANHANES: a unique survey series in the health landscape. S Afr Med J 2014 104: 675-76.

203 Atun R, Gale EAM. The challenge of diabetes in sub-Saharan Africa. Lancet Diabetes Endocrinol 2015; 3: 675-77.

204 Bommer C, Heesemann E, Sagalova V, et al. The global economic burden of diabetes in adults aged 20-79 years: a cost-of-illness study. Lancet Diabetes Endocrinol 2017; 5: 423-30.

205 Chale SS, Swai AB, Mujinja PG, McLarty DG. Must diabetes be a fatal disease in Africa? Study of costs of treatment. BMJ 1992; 304: 1215-18.

206 Nkegoum AV. Coût direct et indirect du diabéte en l'absence de complications chroniques à Yaoundé, Cameroun. MD thesis, University of Yaoundé I, 2002.

207 Minor T. An investigation into the effect of type I and type II diabetes duration on employment and wages. Econ Hum Biol 2013; 11: 534-44.

208 Loeppke R, Taitel M, Haufle V, Parry T, Kessler RC, Jinnett K. Health and productivity as a business strategy: a multiemployer study. J Occup Environ Med 2009; 51: 411-28.

209 Seuring T, Goryakin Y, Suhrcke M. The impact of diabetes on employment in Mexico. Econ Hum Biol 2015; 18: 85-100.

210 Seuring T, Serneels P, Suhrcke ME. The impact of diabetes on labour market outcomes in Mexico: a panel data and biomarker analysis. York: Centre for Health Economics, University of York, 2016.

211 Yang W, Dall TM, Halder P, Gallo P, Kowal SL, Hogan PF, for the American Diabetes Association. Economic costs of diabetes in the U.S. in 2012. Diabetes Care 2013; 36: 1033-46.
212 Sharma KM, Ranjani H, Zabetian A, et al. Excess cost burden of diabetes in southern India: a clinic-based, comparative cost-of-illness study. Global Health Epidemiol Genom 2016; 1: e8.

213 Esteghamati A, Khalilzadeh O, Anvari M, et al. The economic costs of diabetes: a population-based study in Tehran, Iran. Diabetologia 2009; 52: 1520-27.

214 Guariguata L, de Beer I, Hough R, et al. Diabetes, HIV and other health determinants associated with absenteeism among formal sector workers in Namibia. BMC Public Health 2012; 12: 44.

215 Kirigia JM, Sambo HB, Sambo LG, Barry SP. Economic burden of diabetes mellitus in the WHO African region. BMC Int Health Hum Rights 2009; 9: 6.

216 UN Department of Economic and Social Affairs, Population Division. The World population prospects: the 2015 revision. 2015. CD-ROM edition.

217 UN Department of Economic and Social Affairs, Population Division. World urbanization prospects: the 2014 revision. CD-ROM edn, 2014.

218 Assah FK, Ekelund U, Brage S, Mbanya JC, Wareham NJ Urbanization, physical activity, and metabolic health in sub-Saharan Africa. Diabetes Care 2011; 34: 491-96.

219 OECD. Input costs for health care provision. https://stats.oecd.org/ Index.aspx?DataSetCode=SHA_FP (accessed June 23, 2017).

220 UK Prospective Diabetes Study Group. Tight blood pressure control and risk of macrovascular and microvascular complications in type 2 diabetes: UKPDS 38. BMJ 1998; 317: 703-13.

221 Duckworth W, Abraira C, Moritz T, for the VADT Investigators. Glucose control and vascular complications in veterans with type 2 diabetes. N Engl J Med 2009; 360: 129-39.

222 Sussman J, Vijan S, Hayward R. Using benefit-based tailored treatment to improve the use of antihypertensive medications. Circulation 2013; 128: 2309-17

223 Basu S, Yudkin JS, Sussman JB, Millett C, Hayward RA. Alternative strategies to achieve cardiovascular mortality goals in China and India: a microsimulation of target- versus risk-based blood pressure treatment. Circulation 2016; 133: 840-48.

224 Crampin AC, Dube A, Mboma S, et al. Profile: the Karonga Health and Demographic Surveillance System. Int J Epidemiol 2012; 41: $676-85$

225 Govindasamy D, Kranzer K, van Schaik N, et al. Linkage to HIV, TB and non-communicable disease care from a mobile testing unit in Cape Town, South Africa. PLoS One 2013; 8: e80017.

226 Haffner SM, for the American Diabetes Association. Dyslipidemia management in adults with diabetes. Diabetes Care 2004; 27 (suppl 1): S68-71.

227 WHO. Package of essential noncommunicable (PEN) disease interventions for primary health care in low-resource settings. 2010. http://www.who.int/nmh/publications/essential_ncd_ interventions_lr_settings.pdf (accessed June 12, 2017).

228 Inzucchi SE. Diabetes facts and guidelines: 2011. New Haven, CT: Yale Diabetes Centre, 2011.

229 Salomon JA, Vos T, Hogan DR, et al. Common values in assessing health outcomes from disease and injury: disability weights measurement study for the Global Burden of Disease Study 2010 Lancet 2012; 380: 2129-43.

230 Atun RA, Lennox-Chhugani N, Drobniewski F, Samyshkin YA, Coker RJ. A framework and toolkit for capturing the communicable disease programmes within health systems: tuberculosis control as an illustrative example. Eur J Public Health 2004; 14: 267-73.

231 WHO. Making choices in health: WHO guide to cost-effectiveness analysis. Geneva: World Health Organization, 2003. http://www. who.int/choice/publications/p_2003_generalised_cea.pdf (accessed June 10, 2017).

232 Kowal P, Chatterji S, Naidoo N, et al. Data resource profile: the World Health Organization Study on global AGEing and adult health (SAGE). Int J Epidemiol 2012; 41: 1639-49.

233 Atun RA, Samyshkin YA, Drobniewski F, et al. Barriers to sustainable tuberculosis control in the Russian Federation health system. Bull World Health Organ 2005; 83: 217-23.

234 Shigayeva A, Atun R, McKee M, Coker R. Health systems, communicable diseases and integration. Health Policy Plan 2010; 25 (suppl 1): i4-20. 
235 Atun RA, McKee M, Drobniewski F, Coker R. Analysis of how the health systems context shapes responses to the control of human immunodeficiency virus: case-studies from the Russian Federation. Bull World Health Organ 2005; 83: 730-38.

236 Atun R, Lazarus JV, Van Damme W, Coker R. Interactions between critical health system functions and HIV/AIDS, tuberculosis and malaria programmes. Health Policy Plan 2010; 25 (suppl 1): i1-3.

237 Jenkins R, Lancashire S, McDaid D, et al. Mental health reform in the Russian Federation: an integrated approach to achieve social inclusion and recovery. Bull World Health Organ 2007; 85: 858-66.

238 Atun R, Pothapregada SK, Kwansah J, Degbotse D, Lazarus JV. Critical interactions between the Global Fund-supported HIV programs and the health system in Ghana. J Acquir Immune Defic Syndr 2011; 57 (suppl 2): S72-76.

239 Unwin N, Mugusi F, Aspray T, et al. Tackling the emerging pandemic of non-communicable diseases in sub-Saharan Africa: the essential NCD health intervention project. Public Health 1999; 113: 141-46.

240 Labhardt ND, Balo JR, Ndam M, Manga E, Stoll B. Improved retention rates with low-cost interventions in hypertension and diabetes management in a rural African environment of nurse-led care: a cluster-randomised trial. Trop Med Int Health 2011; 16: 1276-84.

241 Sobry A, Kizito W, Van den Bergh R, et al. Caseload, management and treatment outcomes of patients with hypertension and/or diabetes mellitus in a primary health care programme in an informal setting. Trop Med Int Health 2014; 19: 47-57.

242 Watkins PJ. Delivering care for diabetes in Ethiopia. Trans R Soc Trop Med Hyg 1999; 93: 355-56.

243 Mamo Y, Seid E, Adams S, Gardiner A, Parry E. A primary healthcare approach to the management of chronic disease in Ethiopia: an example for other countries. Clin Med 2007; 7: 228-31.

244 Katz I, Schneider H, Shezi Z, et al. Managing type 2 diabetes in Soweto- the South African Chronic Disease Outreach Program experience. Prim Care Diabetes 2009; 3: 157-64.

245 Distiller LA, Brown MA, Joffe BI, Kramer BD. Striving for the impossible dream: a community-based multi-practice collaborative model of diabetes management. Diabet Med 2010; 27: 197-202.

246 Gill GV, Price C, Shandu D, Dedicoat M, Wilkinson D. An effective system of nurse-led diabetes care in rural Africa. Diabet Med 2008; 25: 606-11.

247 Price C, Shandu D, Dedicoat M, Wilkinson D, Gill GV. Long-term glycaemic outcome of structured nurse-led diabetes care in rural Africa. QJM 2011; 104: 571-74.

248 Kengne AP, Fezeu L, Sobngwi E, et al. Type 2 diabetes management in nurse-led primary healthcare settings in urban and rural Cameroon. Prim Care Diabetes 2009; 3: 181-88.

249 Ndou T, van Zyl G, Hlahane S, Goudge J. A rapid assessment of a community health worker pilot programme to improve the management of hypertension and diabetes in Emfuleni sub-district of Gauteng Province, South Africa. Glob Health Action 2013; 6: 19228.

250 Joubert PH, Sebata PD, Bam WJ, Skene DJ. Home urinary glucose testing. Its impact on a third world diabetic population. S Afr Med J 1984; 65: 731-33.

251 Pastakia SD, Karwa R, Kahn CB, Nyabundi JS. The evolution of diabetes care in the rural, resource-constrained setting of western Kenya. Ann Pharmacother 2011; 45: 721-26.

252 Pastakia SD, Ali SM, Kamano JH, et al. Screening for diabetes and hypertension in a rural low income setting in western Kenya utilizing home-based and community-based strategies. Global Health 2013; 9: 21.

253 Gessler N, Labhard ND, Stolt P, et al. The lesson of Monsieur Nouma: effects of a culturally sensitive communication tool to improve health-seeking behavior in rural Cameroon. Patient Educ Couns 2012; 87: 343-50.

254 van der Does AMB, Mash R. Evaluation of the "Take Five School": an education programme for people with type 2 diabetes in the Western Cape, South Africa. Prim Care Diabetes 2013; 7: 289-95.

255 Mukama LJ, Moran A, Nyindo M, Philemon R, Msuya L. Improved glycemic control and acute complications among children with type 1 diabetes mellitus in Moshi, Tanzania. Pediatr Diabetes 2013; 14: 211-16.

256 Windus DW, Ladenson JH, Merrins CK, et al. Impact of a multidisciplinary intervention for diabetes in Eritrea. Clin Chem 2007; 53: 1954-59.
257 Amoah AGB, Owusu SK, Acheampong JW, et al. A national diabetes care and education programme: the Ghana model. Diabetes Res Clin Pract 2000; 49: 149-57.

258 MakkiAwouda FO, Elmukashfi TA, Hag Al-Tom SA. Designing an educational and training program for diabetes health educators at Diabetic Health Centers, Khartoum State, Sudan; 2007-2010. Glob J Health Sci 2013; 5: 207-11.

259 Kouematchoua Tchuitcheu G, Rienhoff O. Options for diabetes management in sub-Saharan Africa with an electronic medical record system. Methods Inf Med 2011; 50: 11-22.

260 Steyn K, Lombard C, Gwebushe N, et al. Implementation of national guidelines, incorporated within structured diabetes and hypertension records at primary level care in Cape Town, South Africa: a randomised controlled trial. Glob Health Action 2013; 6: 20796.

261 Allain TJ, van Oosterhout JJ, Douglas GP, et al. Applying lessons learnt from the 'DOTS' tuberculosis model to monitoring and evaluating persons with diabetes mellitus in Blantyre, Malawi. Trop Med Int Health 2011; 16: 1077-84.

262 Chamie G, Kwarisiima D, Clark TD, et al, for the SEARCH Collaboration. Leveraging rapid community-based HIV testing campaigns for non-communicable diseases in rural Uganda. PLoS One 2012; 7: e43400.

263 Goudge J, Gilson L, Russell S, Gumede T, Mills A. Affordability, availability and acceptability barriers to health care for the chronically ill: longitudinal case studies from South Africa. BMC Health Serv Res 2009; 9: 75.

264 Haque M, Emerson SH, Dennison CR, Navsa M, Levitt NS Barriers to initiating insulin therapy in patients with type 2 diabetes mellitus in public-sector primary health care centres in Cape Town. S Afr Med J 2005; 95: 798-802.

265 Hjelm K, Atwine F. Health-care seeking behaviour among persons with diabetes in Uganda: an interview study. BMC Int Health Hum Rights 2011; 11: 11

266 Kolling M, Winkley K, von Deden M. “For someone who's rich, it's not a problem". Insights from Tanzania on diabetes health-seeking and medical pluralism among Dar es Salaam's urban poor. Global Health 2010; 6: 8.

267 Gill GV, Yudkin JS, Keen H, Beran D. The insulin dilemma in resource-limited countries. A way forward? Diabetologia 2011; 54: 19-24.

268 Atun R, Jaffar S, Nishtar S, et al. Improving responsiveness of health systems to non-communicable diseases. Lancet 2013; 381: 690-97.

269 Fraser HSF, Allen C, Bailey C, Douglas G, Shin S, Blaya J. Information systems for patient follow-up and chronic management of HIV and tuberculosis: a life-saving technology in resource-poor areas. J Med Internet Res 2007; 9: e29.

270 Shiferaw F, Zolfo M. The role of information communication technology (ICT) towards universal health coverage: the first steps of a telemedicine project in Ethiopia. Glob Health Action 2012; 5: 1-8.

271 Kahn JG, Yang JS, Kahn JS. 'Mobile' health needs and opportunities in developing countries. Health Aff 2010; 29: 252-58.

272 Chandrasekhar CP, Ghosh J. Information and communication technologies and health in low income countries: the potential and the constraints. Bull World Health Organ 2001; 79: 850-55.

273 Vodopivec-Jamsek V, de Jongh T, Gurol-Urganci I, Atun R, Car J. Mobile phone messaging for preventive health care. Cochrane Database Syst Rev 2012; 12: CD007457.

274 Gurol-Urganci I, de Jongh T, Vodopivec-Jamsek V, Car J, Atun R. Mobile phone messaging for communicating results of medical investigations. Cochrane Database Syst Rev 2012: 6: CD007456.

275 de Jongh T, Gurol-Urganci I, Vodopivec-Jamsek V, Car J, Atun R. Mobile phone messaging telemedicine for facilitating self-management of long-term illnesses. Cochrane Database Syst Rev 2008; 4: CD007459.

276 Car J, Gurol-Urganci I, de Jongh T, Vodopivec-Jamsek V, Atun R. Mobile phone messaging reminders for attendance at healthcare appointments. Cochrane Database Syst Rev 2012; 7: CD007458.

277 van Velthoven MH, Tudor Car L, Car J, Atun R. Telephone consultation for improving health of people living with or at risk of HIV: a systematic review. PLoS One 2012; 7: e36105.

278 Palmer JJ, Chinanayi F, Gilbert A, et al. Mapping human resources for eye health in 21 countries of sub-Saharan Africa: current progress towards VISION 2020. Hum Resour Health 2014; 12: 44. 
279 Hansen MB, Abràmoff MD, Folk JC, Mathenge W, Bastawrous A, Peto T. Results of automated retinal image analysis for detection of diabetic retinopathy from the Nakuru Study, Kenya. PLoS One 2015 10: $\mathrm{e} 0139148$.

280 Dyck PJ, Overland CJ, Low PA, et al. Signs and symptoms versus nerve conduction studies to diagnose diabetic sensorimotor polyneuropathy: Cl vs NPhys trial. Muscle Nerve 2010; 42: 157-64.

281 Atun R, de Jongh T, Secci F, Ohiri K, Adeyi O. Integration of targeted health interventions into health systems: a conceptual framework for analysis. Health Policy Plan 2010; 25: 104-11.

282 Atun R. Health systems, systems thinking and innovation. Health Policy Plan 2012; 27 (suppl 4): iv4-8.

283 van Olmen J, Schellevis F, Van Damme W, Kegels G, Rasschaert F. Management of chronic diseases in Sub-Saharan Africa: cross-fertilisation between HIV/AIDS and diabetes care. J Trop Med 2012; 2012: 349312.

284 Schouten EJ, Jahn A, Midiani D, et al. Prevention of mother-to-child transmission of HIV and the health-related Millennium Developmen Goals: time for a public health approach. Lancet 2011; 378: 282-84.

285 WHO. NCD global monitoring framework. http://www.who.int/ $\mathrm{nmh}$ /global_monitoring_framework/en (accessed March 10, 2017).

286 Popkin BM, Hawkes C. Sweetening of the global diet, particularly beverages: patterns, trends, and policy responses. Lancet Diabetes Endocrinol 2016; 4: 174-86.

287 PSI. Why PSI uses social marketing: the evidence base. Washington, DC: Population Sciences International, 2011 http://www.psi.org/wp-content/uploads/drupal/sites/default/files/ publication_files/SM-Evidence-Base_March2011-update.pdf (accessed June 12, 2017).

288 Mauer M, Zinman B, Gardiner R, et al. Renal and retinal effects of enalapril and losartan in type 1 diabetes. N Engl J Med 2009; 361: 40-51.

289 UN. Declaration of commitment on HIV/AIDS. "Global crisisglobal action". http://www.un.org/ga/aids/coverage/ FinalDeclarationHIVAIDS.html (accessed March 10, 2017).

290 UN. Political declaration of the high-level meeting of the General Assembly on the prevention and control of non-communicable diseases. 2011. http://www.un.org/ga/search/view_doc. asp?symbol=A/66/L.1 (accessed March 10, 2017).

291 Swinburn BA, Sacks G, Hall KD, et al. The global obesity pandemic: shaped by global drivers and local environments. Lancet 2011; 378: 804-14
292 UN. Resolution adopted by the General Assembly on 13 May 2010 64/265. Prevention and control of non-communicable diseases. 2010. http://www.un.org/en/ga/search/view_doc.asp?symbol=A/ RES/64/265\&Lang=E (accessed March 10, 2017).

293 WHO. Sixty-fifth World Health Assembly. Second report of Committee A. http://apps.who.int/gb/ebwha/pdf_files/WHA65/ A65_54-en.pdf (accessed March 10, 2017).

294 Atun R. Decisive action to end apathy and achieve $25 \times 25$ NCD targets. Lancet 2014; 384: 384-85.

295 Moon S, Sridhar D, Pate MA, et al. Will Ebola change the game? Ten essential reforms before the next pandemic. The report of the Harvard-LSHTM Independent Panel on the Global Response to Ebola. Lancet 2015; 386: 2204-21.

296 Jobanputra K, Boulle P, Roberts B, Perel P. Three steps to improve management of noncommunicable diseases in humanitarian crises. PLoS Med 2016; 13: e1002180.

297 Evans T, Nishtar S, Atun R, Etienne C. Scaling up research and learning for health systems: time to act. Lancet 2008; 372: 1529-31.

298 Beran D, Byass P, Gbakima A, et al. Research capacity buildingobligations for global health partners. Lancet Glob Health 2017: 5: e567-68.

299 George PP, Papachristou N, Belisario JM, et al. Online eLearning for undergraduates in health professions: a systematic review of the impact on knowledge, skills, attitudes and satisfaction. $J$ Glob Health 2014; 4: 010406.

300 de Jongh T, Gurol-Urganci I, Vodopivec-Jamsek V, Car I, Atun R Mobile phone messaging for facilitating self-management of long term illnesses. Cochrane Database Syst Rev 2012; 12: CD007459.

301 Atun R, Knaul FM, Akachi Y, Frenk J. Innovative financing for health: what is truly innovative? Lancet 2012; 380: 2044-49.

302 Atun R, Silva S, Ncube M, Vassall A. Innovative financing for HIV response in sub-Saharan Africa. J Glob Health 2016; 6: 010407.

303 Fitchett JR, Fan Li J, Atun R. Innovative financing for late-stage global health research and development: the Global Health Investment Fund. Int Health 2016; 8: 3-4. 